\title{
Structure and biochemistry of polyunsaturated fatty acid double bond isomerase from Propionibacterium acnes
}

\author{
PhD Thesis \\ In partial fulfillment of the requirements \\ for the degree "Doctor of Philosophy (PhD)" \\ in the Molecular Biology Program \\ at the Georg August University Göttingen, \\ Faculty of Biology
}

Submitted by

Alena Liavonchanka

Born in Vysoki Borak, Belarus 


\section{Affidavit}

Hereby I declare that my thesis entitled "Structure and biochemistry of polyunsaturated fatty acid double bond isomerase from Propionibacterium acnes" has been written independently and with no other sources and aids than quoted.

Alena Liavonchanka

Göttingen, 28.09.2007 
To all my teachers 


\section{List of publications}

Liavonchanka, A., and Feussner, I. (2006) Lipoxygenases: occurrence, functions and catalysis. J Plant Physiol 163, 348-357.

Liavonchanka, A., Hornung, E., Feussner, I., and Rudolph, M. (2006) In-house SIRAS phasing of the polyunsaturated fatty-acid isomerase from Propionibacterium acnes. Acta Crystallograph Sect F Struct Biol Cryst Commun 62, 153-156.

Liavonchanka, A., Hornung, E., Feussner, I., and Rudolph, M. G. (2006) Structure and mechanism of the Propionibacterium acnes polyunsaturated fatty acid isomerase. Proc Natl Acad Sci U S A 103, 2576-2581. 


\section{Table of contents}

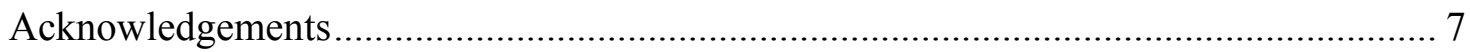

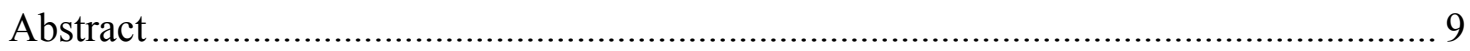

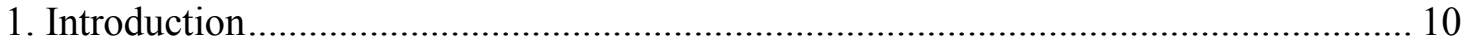

1.1 Physiological action of CLA and possible mechanisms ........................................ 11

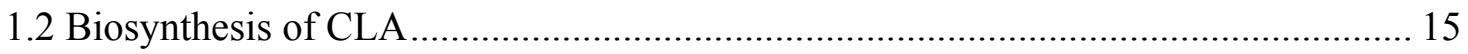

1.3 Comparative analysis of enzymatic mechanisms involved in carbon-carbon double

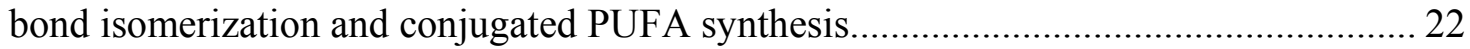

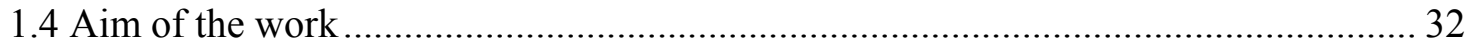

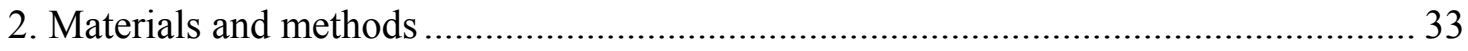

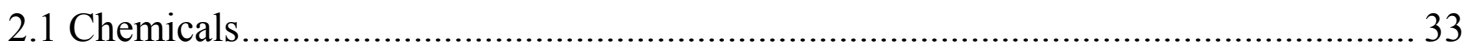

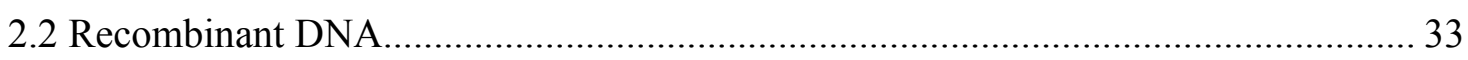

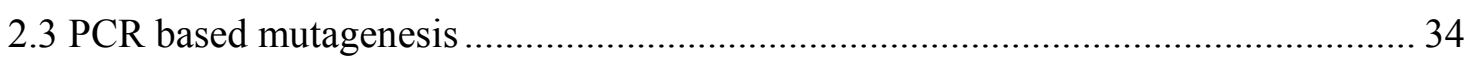

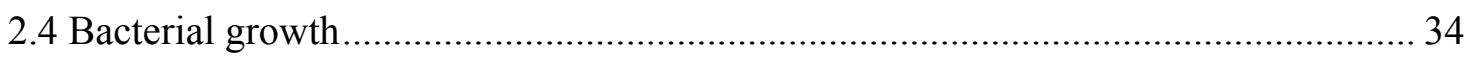

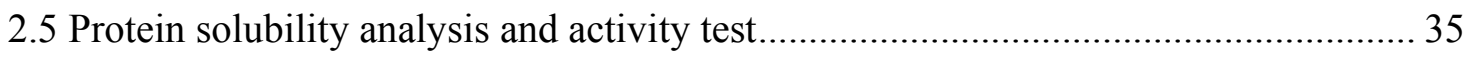

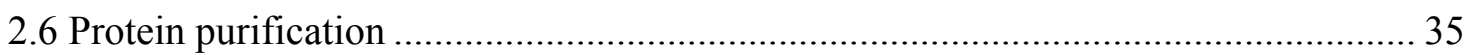

2.6.1 Affinity chromatography on Glutathione Sepharose ….......................................... 35

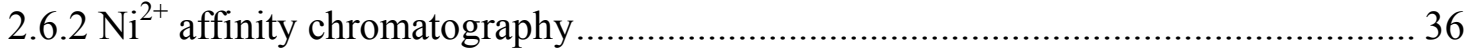

2.6.3 Ion exchange chromatography on Source 30Q Sepharose ..................................... 36

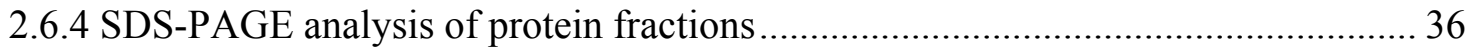

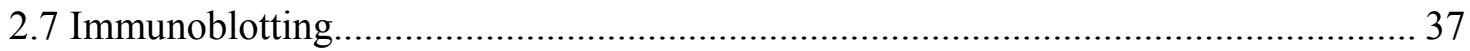

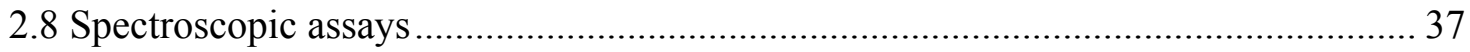

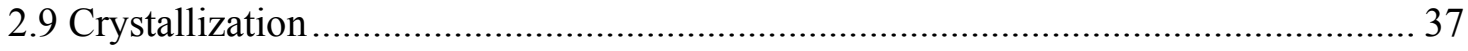

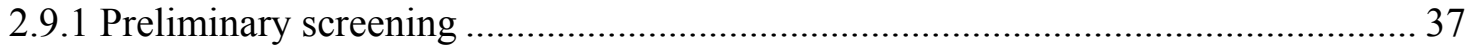

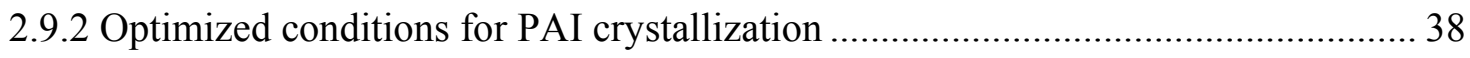

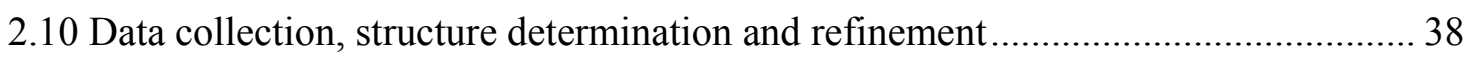

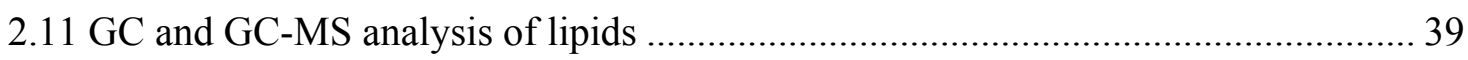

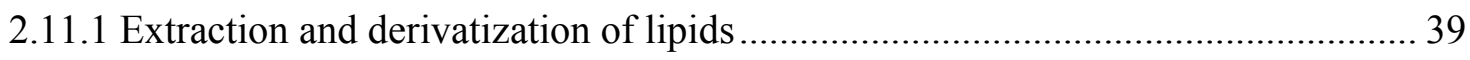

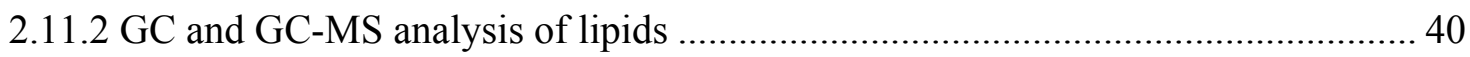

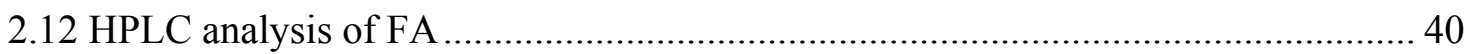

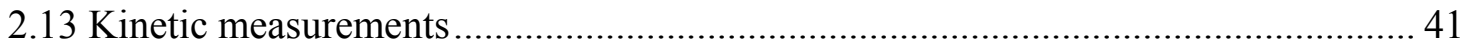

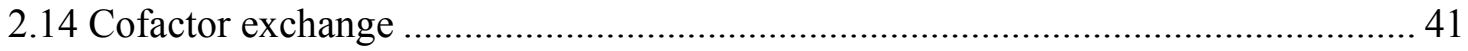

2.15 Anaerobic photoreduction and redox potential measurement ................................ 42 
2.17 MALDI TOF analysis 42

3. Results 45

3.1 PAI structure. 45

3.1.1 PAI purification and biochemical properties ..... 45

3.1.2 Crystallization, data collection, structure determination, and refinement 45

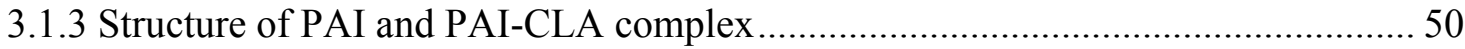

3.1.3.1 Overall structure description of free PAI........................................................... 50

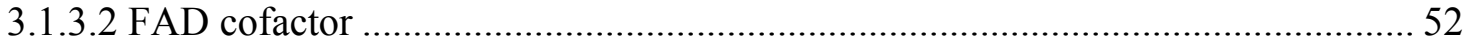

3.1.3.3 Structure of PAI-CLA and PAI-CLnA complexes ............................................. 53

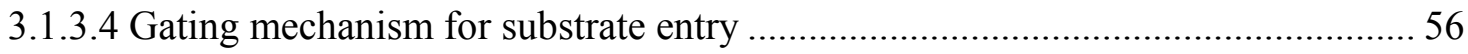

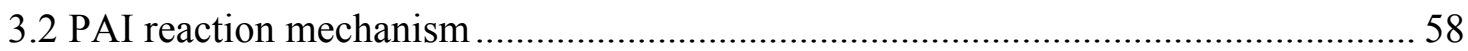

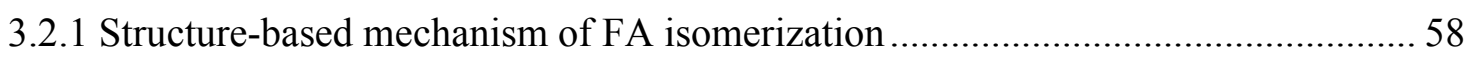

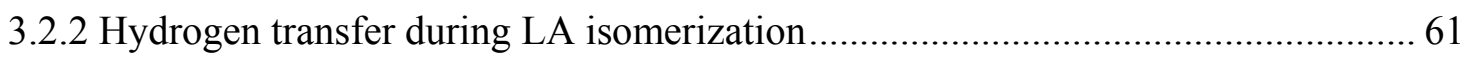

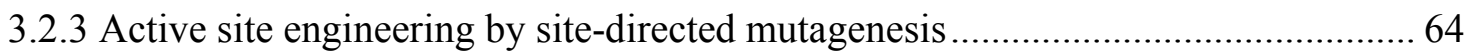

3.2.4 Enzyme-substrate complex formation, anaerobic reduction, cofactor exchange .... 66

3.3 FA hydratase from Streptococcus pyogenes $(\mathrm{SPH})$.................................................. 70

3.3.1 Cloning, purification and spectral properties ........................................................ 70

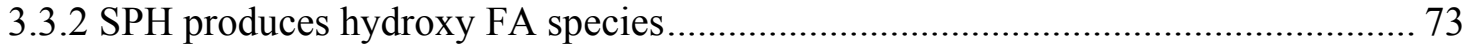

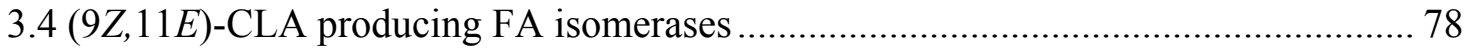

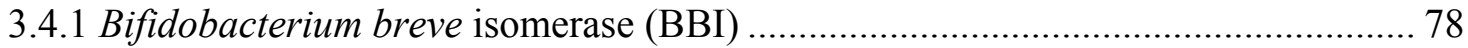

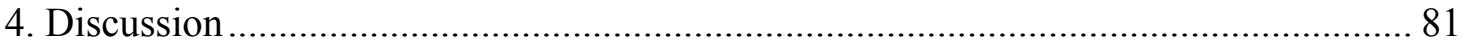

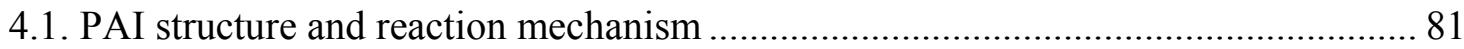

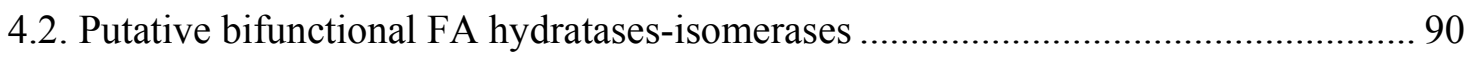

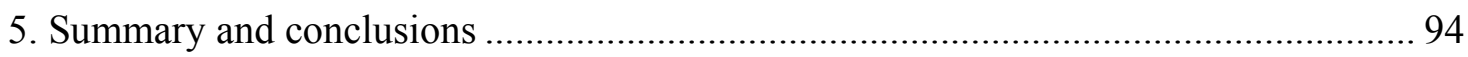

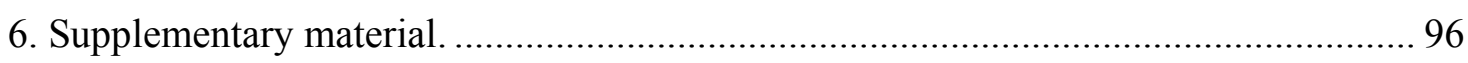

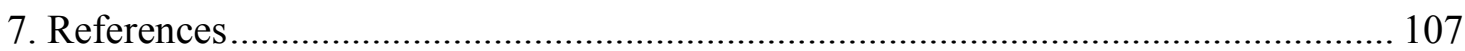

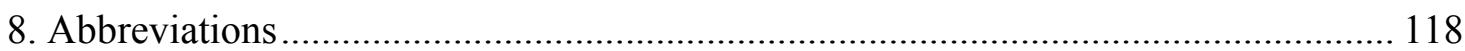

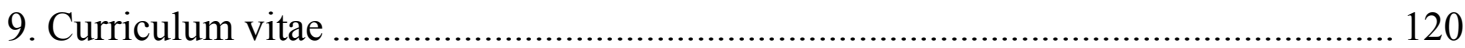




\section{List of figures}

Fig. 1 Structures of LA, (9Z,11E)-CLA and (10E,12Z)-CLA...................................... 11

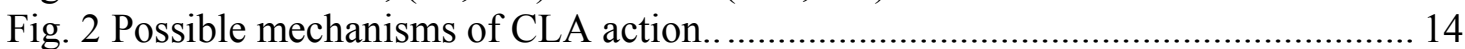

Fig. 3 Structures of EPA and conjugated triene product produced by PFI..................... 18

Fig. 4 Scheme of LA conversion to hydroxy FAs by Lactobacillus sp ........................... 19

Fig.5 The routes of LA metabolism in anaerobic bacteria of human gut. ....................... 21

Fig. 6 Schematic overview of enzymatic mechanisms for carbon-carbon double bond

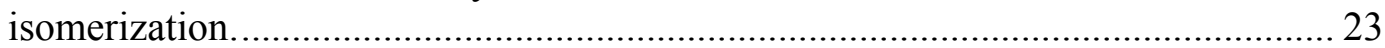

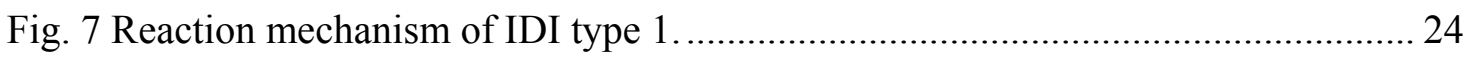

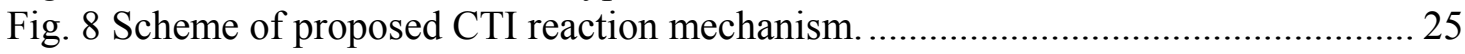

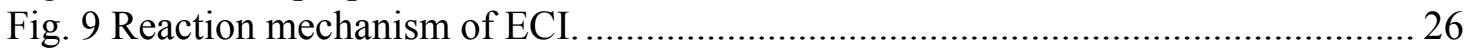

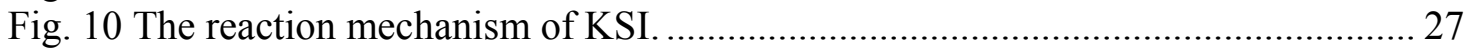

Fig. 11 Analysis of active site geometry in FabA and FabZ....................................... 28

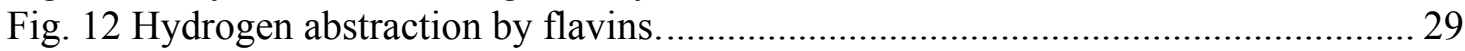

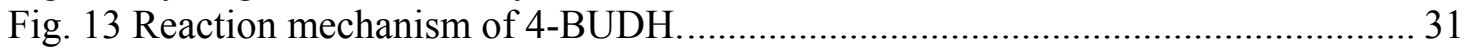

Fig. 14 PAI purification, spectral properties and crystallization. ................................. 47

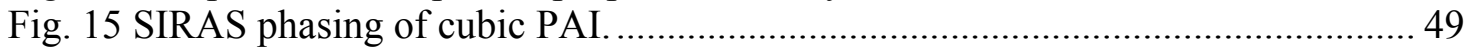

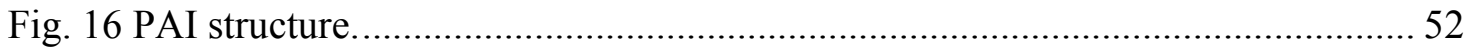

Fig. 17 Sequence conservation between PAI, PFI and polyamine oxidase...................... 52

Fig. 18 Contacts of CLA (a) and FAD (b) in the active site.......................................... 53

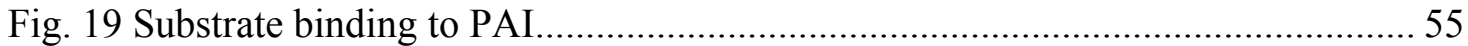

Fig. 20 Analysis of the fatty acid content in PAI crystals obtained after co-crystallization

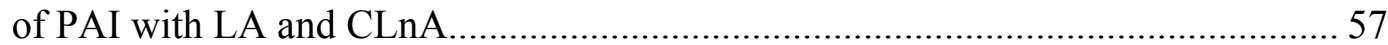

Fig. 21 Substrate entry channel and gating mechanism in PAI.................................... 59

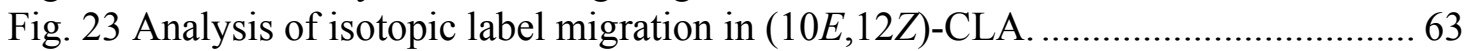

Fig. 24 Overexpression of LOX-PAI fusions and PAI point mutants. ................................6 66

Fig. 25 Spectral changes upon PAI-CLA complex formation and PAI reduction............. 67

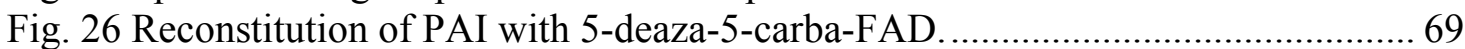

Fig. 27 Sequence alignment of putative PUFA double bond isomerases ........................ 71

Fig. 28 Purification and properties of SPH and BBI. .................................................... 72

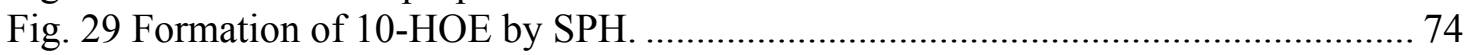

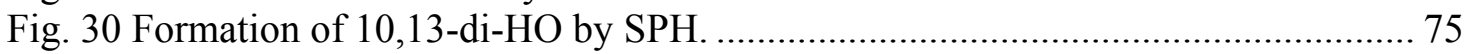

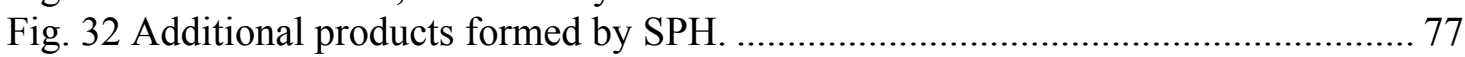

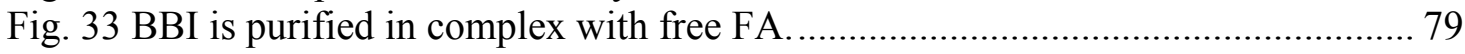

Fig. 34 Stereochemistry of hydrogen transfer in PUFA isomerases................................ 87

\section{List of tables}

Table 1. Data collection, phasing and refinement statistics.........................43

Table 2. Kinetic parameters of PAI wt and mutant forms. 


\section{Acknowledgements}

I thank my PhD committee members Prof. I. Feußner, Dr. M. Rudolph and Prof. C. Griesinger for the constant input of ideas and productive project evaluation. I would like to express my gratitude in particular to Prof. Feußner for providing me a large degree of freedom in research directions and Dr. Rudolph for the inspirational introduction to the X-ray crystallography field.

I am grateful to Dr. C. Göbel for continuous support and advise related to analytical techniques. The experiments lead by Dr. E. Hornung in many aspects laid the basis of the present work. I also thank Prof. O. Einsle for providing the hardware for the redox experiments and for the discussion of experimental data, Dr. M. Hoffman and P. Lukat for the practical help with anaerobic techniques. I thank Prof. R. Ficner for providing me the opportunity to work in the department of Molecular Structural Biology as a guest student. This work would not be complete without generous gifts of Prof. S. Ghisla (5deaza-5-carba-FAD) and Prof. M. Hamberg (isotopically labeled linoleic acid). The mass-spectrometric analysis of protein samples was performed at the Bioanalytical Mass Spectrometry division, Max-Planck Institute for Biophysical Chemistry, Goettingen, lead by Dr. H. Urlaub.

It was a great experience for me to supervise my colleague students from IMPRS Molecular Biology Magdalena Moravska, Katharina Hoppe and Hanna Peradziryj in the frame of lab rotation trainings performed in our department. Finally, I thank the IMPRS for Molecular Biology for unforgettable four years I spent in Göttingen as a member of MolBio program and the State of Lower Saxonia for the financial support in form of a Georg-Christoph Lichtenberg stipend. 


\begin{abstract}
Conjugated linoleic acid (CLA) is a collective term describing positional and geometrical isomers of linoleic acid (LA, 18:2 $2^{\Delta 9 Z, 12 Z}$ ). This class of compounds is receiving much attention in the field of lipid research due to its broad beneficial effects on human health, including normalization of body fat content, immunomodulatory and anti-carcinogenic properties. The major gaps in understanding of CLA biosynthesis exist up to date, impeding the efficient industrial production of pure CLA isomers.

The present work describes the reaction mechanism of CLA producing isomerase from Propionibacterium acnes (PAI), based on the atomic structure of PAI as a free protein and in complex with its CLA product. PAI was crystallized as monomeric protein and the structure was determined by X-ray crystallography. Each PAI monomer contains one molecule of non-covalently bound FAD, which acts as redox catalyst during the isomerization of LA. The enzyme recognizes the free carboxylic group of LA by polar interactions with two residues, $\operatorname{Arg} 88$ and Phe193, which act as a lock at the entrance to the active site. The transfer of pro- $R$-hydrogen from the position C11 of LA to the position $\mathrm{C} 9$ was predicted based on the structural data and confirmed by mass spectrometric analysis of isotopically labeled LA derivatives. FAD radical is likely to form during PAI turnover, as deduced from spectroscopic data and cofactor exchange. PAI represents the first structure of a fatty acid double bond isomerase, providing the framework for characterization of related enzymes. Three of such putative CLA producing enzymes, distantly related to PAI, were shown to hydrate LA, forming hydroxy derivatives, which indicates that an alternative pathway for CLA biosynthesis exists in bacteria of Lactobacillus, Bifidobacterium and Streptococcus species.
\end{abstract}




\section{Introduction}

Fatty acids (FA) are playing a central role in the metabolism as energy storing units and major building blocks for cellular membranes. Apart from these fundamental functions FAs are also precursors for signaling molecules and hormones. The discovery of eicosanoids (Samuelsson 1987), the products of arachidonic acid (AA) oxidation by mammalian enzymes prostaglandin endoperoxide H synthase (PGHS) or 5-lipoxygenase (5-LOX), opened the new era in inflammation therapy. On the other hand, a number of linolenic acid (LnA) metabolites collectively called jasmonates regulate many aspects of plant growth, development and response to pathogens (Turner et al. 2002). Relatively recently, a new class of LA derivatives, abbreviated as conjugated linoleic acid (CLA), has drawn attention as potentially useful anti-carcinogenic substance (Ha et al. 1987). The term CLA was introduced to describe the increasing number of positional and geometrical isomers of LA possessing a conjugated double bond system. Formation of $(9 Z, 11 E)$-CLA by ruminant bacteria was described almost twenty years before its anticarcinogenic effect was demonstrated, and the latter finding revived the interest in the CLA research.

Predominant pathway of CLA biosynthesis is so called biohydrogenation performed by ruminant bacteria (Yurawecz 1999). The anaerobic bacteria of rumen are able to reduce double bonds of polyunsaturated FAs (PUFAs) during the fermentation and CLA is one of the intermediate products (see below). The data accumulated up to date demonstrate that mainly two CLA isomers, namely (9Z,11E)-CLA and (10E,12Z)-CLA elicit multiple physiological effects in humans when consumed in the amounts 3-7 g/day (Pariza 2004, Wahle et al. 2004). These effects include anti-carcinogenic, immunomodulatory, anti-inflammatory, reduction of body fat and symptoms of astma, diabetes and atherosclerosis (Wahle et al. 2004). The structures of both isomers and the parent LA molecule are shown in Fig. 1. The wide range of responses implies that no single receptor or signaling pathway underlies CLA effects, rather the multiple aspects of cell homeostasis and metabolism are perturbed (Pariza et al. 2000). Moreover, it appears that some of the responses to CLA are isomer-specific and data from animal studies are not always applicable to human physiology (Pariza 2003). The current knowledge about effects of CLA on cellular signaling pathways and human physiology is far from being 


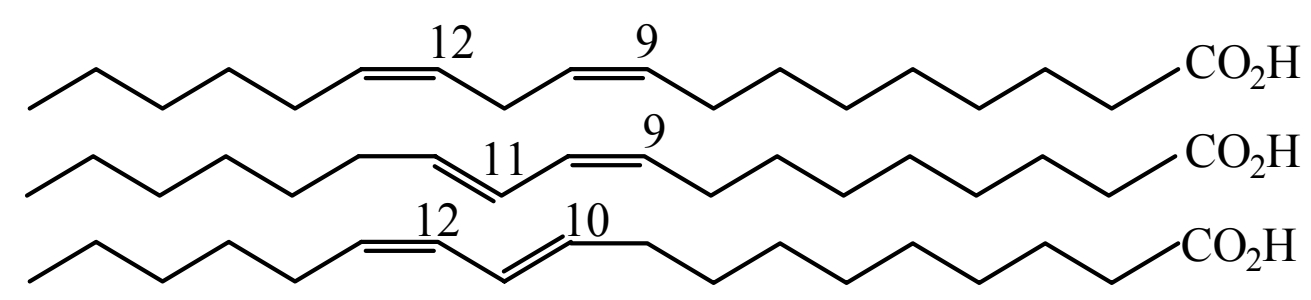

Fig. 1 Structures of LA, $(9 Z, 11 E)$-CLA and (10E,12Z)-CLA (top to bottom). Double bond positions are indicated by numbers.

complete and is briefly summarized in the next section. The main focus of the present work was on biochemical mechanisms of CLA synthesis in bacterial systems, therefore the available data on known CLA-producing and related enzymes are reviewed in greater detail later on.

\subsection{Physiological action of CLA and possible mechanisms}

It appears that at least some aspects of body fat mass regulation can be specifically attributed to (10E,12Z)-CLA (Pariza 2004). Mice are most susceptible to CLA among experimental animals and in this species the correlation between body fat mass and (10E,12Z)-CLA uptake was shown in several studies (Park et al. 1997, Park et al. 1999). Specifically, the accretion of body fat is inhibited rather than decrease in already present fat tissue (Pariza et al. 2001). (10E,12Z)-CLA was shown to inhibit adipocyte lipoprotein lipase activity thus preventing lipid uptake by adipocytes (Park et al. 1999, Park et al. 2004 ) and to increase FA oxidation in cultured 3T3-L1 preadipocytes (Evans et al. 2002). In addition, the maturation and differentiation of adipocytes is reduced by $(10 E, 12 Z)$ CLA (Granlund et al. 2005). Recent meta-analysis of clinical studies with human volunteers confirmed the moderate fat loss caused by pure (10E,12Z)-CLA or by the mix of both isomers when administered for four and more weeks (Whigham et al. 2007), however in some clinical studies no correlation between (10E,12Z)-CLA uptake and fat loss was found (Larsen et al. 2003). These discrepancies indicate that direct projection of results from mice model experiments to human physiology is not reasonable (Pariza 2004, Wahle et al. 2004). 
The mechanisms of CLA-dependent immunostimulation and inflammation decrease are even less evident. CLA seems to interfere with early events during immune response such as cytokines release (Yang \& Cook 2003, Yu et al. 2002) and prostaglandin production (Ringseis et al. 2006, Ma et al. 2002, Shen et al. 2004). AA is the precursor of prostaglandins and availability of AA to 5-LOX and PGHS can be altered depending on the presence of other PUFAs (Dinarello 1999). In the cell CLA preferentially accumulates in triacylglycerols (TAGs) and a small fraction of $(9 Z, 11 E)$-CLA was found in the phoshpholipids (PL) (Banni et al. 2004). In addition, the distribution of CLA in different lipid classes is isomer-specific and the metabolic fates of (10E,12Z)-CLA and $(9 Z, 11 E)$-CLA differ in a way that the former is mainly desaturated to $18: 3^{\Delta 6,10,12}$, whereas the latter can be elongated to 20:3 and 20:4 species (Banni et al. 2004, Park et al. 2005).

$(9 Z, 11 E)$-CLA was shown to inhibit the release of pro-inflammatory cytokine tumor necrosis factor alpha (TNF- $\alpha$ ) in rats (Akahoshi et al. 2004) by yet unknown mechanism. Potentially involved mediators are peroxisome proliferator-activated receptor (PPARs) transcription factors, among which PPAR $\gamma$ is known to down-regulate the production of inflammatory cytokines. The interaction of PPAR $\gamma$ and CLA was demonstrated in vitro in macrophage-derived RAW cells treated with interferon-gamma (IFN gamma) (Yu et al. 2002). Another major transcription factor involved in the stress-stimulated signaling cascade is nuclear factor kappa beta (NF-kB), which is normally inactivated by inhibitor subunit IkB. Phosphorylation of IkB mediated by extracellular signal related kinase (ERK) leads to its ubiquitination and proteosomal degradation, releasing free NF-kB, which is then translocated to the nucleus. NF-kB enhances transcription of genes encoding for cytokines, adhesion molecules and heat-shock proteins. Loscher et al. reported that treatment of murine dendritic cells (DC) with $(9 Z, 11 E)$-CLA suppressed lipopolysacharide (LPS)-induced interleukin (IL-12) production, which was concomitant with delayed translocation of NF-kBp65 into the nucleus and an increase in IkB $\alpha$. This suppression was dependent on activation of ERK and enhanced IL-10 production at the transcriptional and protein level (Loscher et al. 2005).

Down-regulation of NF-kB signaling by topical application of $(9 Z, 11 E)$-CLA was also shown to delay mouse skin cancer development (Hwang et al. 2007). This inhibitory 
effect of $(9 Z, 11 E)$-CLA was attributed to the decreased catalytic activity of IkB kinase (IKK). In a mouse prostate tumor model $(9 Z, 11 E)$-CLA significantly increased TNF- $\alpha$ induced apoptosis which correlated with a reduction in NF-kB transcriptional activity, NF-kB binding activity, and phosphorylation of IkB (Song et al. 2006). Influence of CLA on human breast and prostate cancer cells was also studied by group of Wahle (Wahle \& Heys 2002) and clear pro-apoptotic effect was demonstrated. (10E,12Z)-CLA was inducing apoptosis in mouse mammary tumor cells and simultaneously reducing cell proliferation rate; in this model neither LA nor $(9 Z, 11 E)$-CLA showed pro-apoptotic activity (Kim et al. 2005). Remarkably, the action of (10E,12Z)-CLA was related to the reduced levels of 5-LOX metabolite, 5-hydroxy eicosatetraenoic acid (5-HETE), and adding 5-HETE back to tumor cells reduced the (10E,12Z)-CLA effect on both apoptosis and cell proliferation, suggesting that indeed CLA is inhibiting AA metabolism by 5 LOX.

Regardless of multiple beneficial effects of CLA the issue of long-term safety and side effects was brought to light by many research groups. The major concern about obesity treatment with $(10 E, 12 Z)-C L A$ is induction of a so called "fatty liver" or steatosis in the mouse model by this CLA isomer. Javadi et al. monitored the activities of key enzymes of FA synthesis after mice were fed three or twelve weeks with a 1:1 mixture of (10E,12Z)- and $(9 Z, 11 E)$-CLA isomers (Javadi et al. 2004). It was concluded that prolonged, but not short-term, feeding mice with CLA increased hepatic FA synthesis relative to oxidation, despite the decrease in total body fat. Generally, the fatty liver syndrome induced by CLA seems to be limited to mice models. In fact, recent study on rats which were subjected to high-fat diet to induce obesity and hepatic steatosis, showed that treatment with a CLA mix reduced hepatic lipid accumulation without affecting overall adiposity (Purushotham et al. 2007). Another potentially dangerous side-effect of CLA treatment is resistance to insulin. Again, this effect is mostly observed in rodents and currently it seems that $(10 E, 12 Z)$-CLA is associated with greater insulin resistance, and that a CLA mixture increases sensitivity to insulin (Taylor \& Zahradka 2004). The link between insulin sensitivity and trans-FA consumption in humans was recently reviewed (Riserus 2006). This survey of current studies shows no significant effect of trans-FA on insulin sensitivity in lean healthy subjects, which is not the case in insulin 
resistant or diabetic individuals. This is even more pronounced in case of CLA, which clearly impairs insulin sensitivity. In several clinical trials no enhanced insulin resistance caused by CLA was observed in human volunteers (Kamphuis et al. 2003, Kamphuis et al. 2003), therefore the potential benefits of CLA consumption in diabetes treatment should be carefully considered.

The oxidative stress and unfavorable changes in blood lipids were attributed to CLA intake in clinical studies of metabolic syndrome and obesity (Riserus et al. 2002). The supplementation with $(10 E, 12 Z)$-CLA markedly increased lipid peroxidation and the level of C-reactive protein. In a similar study the same group found analogous effects of $(9 Z, 11 E)$-CLA in obese men with high risk of cardiovascular disease (Riserus et al. 2004). The apparent oxidative stress and insuline resistance caused by both CLA isomers need to be confirmed in studies on healthy non-obese subjects and the role of eicosanoids in these symptoms should be clarified.

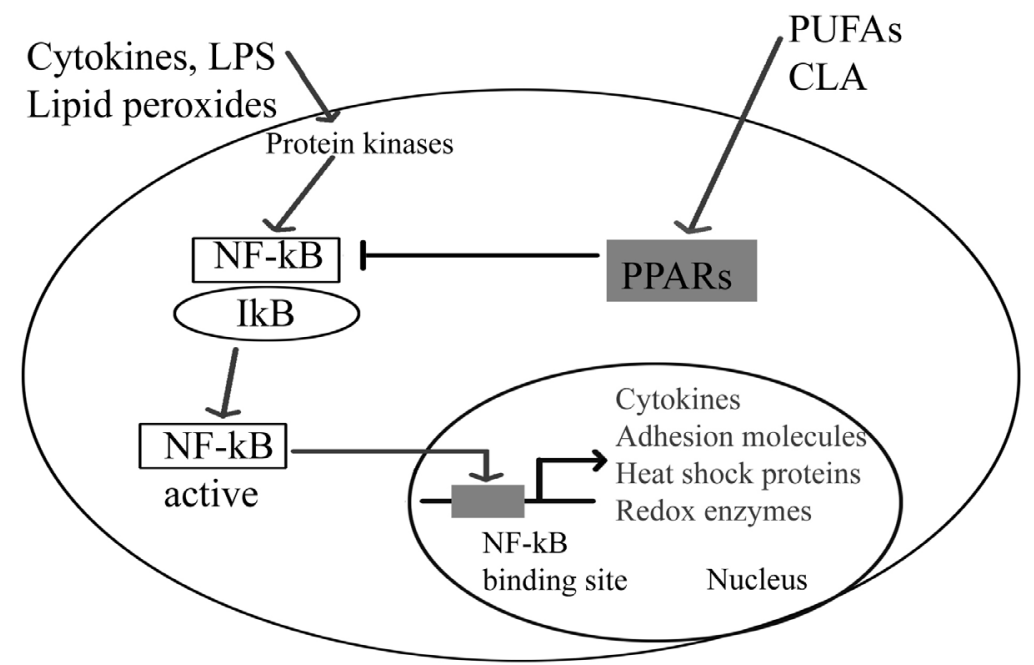

Fig. 2 Possible mechanisms of CLA action. From (Wahle et al. 2004).

Stress-stimuli elicit a signal cascade, activating NF-kB/IkB complex in the cytoplasm and thus releasing active NF-kB. NF-kB then translocates to the nucleus and binds specific $\mathrm{kB}$ response elements in the promoter regions of various genes. These include genes for adhesion molecules, cytokines, redox enzymes, heat shock proteins, cyclooxygenases etc. PPAR activation CLAs may also play a role in regulating NF-kB activity, in addition $\omega-3$ PUFAs can be converted to CLA analogs in the cell and interfere with eicosanoid signaling. 
Taking into account the complexity of prostanoid signaling pathways and a wide array of downstream transcription factors and target genes, it is not surprising that CLA elicits such broad responses in vivo and in vitro. It is now generally accepted that both CLA isomers can regulate the first steps of central stress-response and apoptotic pathways (Wahle et al. 2004, Yurawecz 2006) as illustrated in Fig. 2. The regulation of body fat accumulation and homeostasis is mainly attributed to $(10 E, 12 Z)-C L A$. Up to date, no direct interaction between CLA and a single receptor or transcription factor was demonstrated, raising the need for more detailed studies at the molecular level to confirm or disprove prostanoid-mediated model of CLA action

\subsection{Biosynthesis of CLA}

The main source of CLA in human diet is milk and meat of ruminant animals. Ruminal bacteria as well as strains isolated from human gut produce several CLA isomers (Devillard et al. 2007, Wallace et al. 2007). (9Z,11E)-CLA is further reduced to vaccenic acid (VA, 18:1 $1^{\Delta 11 E}$ ) which is the most abundant trans-FA in ruminant fat products (Yurawecz 2006). Further on, it was shown that VA can be converted back to $(9 Z, 11 E)$-CLA by the action of $\Delta 9$-desaturase in cows (Griinari et al. 2000) and humans, in the latter case the average conversion rate was estimated to be $19 \%$ (Kuhnt et al. 2006).

As early as in 1951 Reiser reported that linseed oil emulsions incubated with rumen contents showed a decrease in LnA content with a corresponding increase in LA, indicating the hydrogenation of LnA to LA (Reiser 1951). The first detailed study on a PUFA double bond isomerase acitivity isolated from Butirivibrio fibrisolvens was published by the group of Tove in a series of papers dated back to 1966-1971 (Kepler et al. 1966, Kepler \& Tove 1967, Kepler et al. 1970, Kepler et al. 1971).

Initially, it was shown that biohydrogenation is not a one-step reduction of LA to VA, rather the enzymatic pathway involves the production of $(9 Z, 11 E)$-CLA accompanied by small amounts of $(9 E, 11 Z)$-CLA and $(9 E, 11 E)$-CLA as intermediates (Kepler et al. 1966). Two trans-monoenoic acids, VA and $(9 E)$-oleic acid $\left(\mathrm{OA}, 18: 1^{\triangle 9 Z}\right)$ were formed from LA as well as from synthetic CLA mix. The rate of CLA formation was found to be much faster than the next step - the reduction of one double bond by iron-dependent reductase (Hughes et al. 1982). The CLA reductase required unusual cofactor alpha- 
tocopherolquinol as an electron donor. Recently, the gene encoding for B. fibrisolvens CLA reductase was cloned and sequence analysis showed no significant similarities to any protein family (Fukuda et al. 2007). Using crude BFI preparation, it was demonstrated that isomerase activity was not affected by any common nucleotide cofactors or metal ions and also was independent on the presence of oxygen. LnA and $\gamma$ LnA were also isomerized to $(9 Z, 11 E, 15 Z)$-CLnA and $(6 Z, 9 Z, 11 E)$-CLnA, respectively (Kepler \& Tove 1967, Kepler et al. 1970). Exclusively the FAs with the $(9 Z, 12 Z)$ position of a pentadiene moiety were BFI substrates, suggesting that BFI "counts" the length from the carboxy-terminus and the first double bond position matters. Any headgroup modification of LA (various esters, amide, hydrazide, hydroxamate, alcohol, methyl ether, methyl ketone, aldehyde) completely abolished the enzymatic activity of BFI.

BFI activity was prone to the substrate inhibition - while the estimated $\mathrm{K}_{\mathrm{m}}$ was 23 $\mu \mathrm{M}$, starting from LA concentration about $50 \mu \mathrm{M}$ the inhibitory effect was evident. (Kepler \& Tove 1967). Most of unsaturated FAs, but not saturated ones, acted as competitive inhibitors of BFI. Concerning the role of the free carboxyl group in inhibition, another series of LA derivatives was tested. One group of substances included methyl linoleate, trilinoleate (TLA), linoleyl aldehyde, linoleyl methyl ketone, and linoleyl methyl ether, none of which caused inhibition. Linoleyl amide, linoleyl hydroxamate, linoleyl hydrazide, linoleyl oxime, linoleyl alcohol, linoleyl amine and linoleyl alcohol tested at the same concentration, inhibited between $30 \%$ and $95 \%$. These experiments clearly indicated the presence of hydrogen bonding between the substrate carboxyl group (and any other head group with polar hydrogen) and the enzyme (Kepler et al. 1970).

When a BFI preparation was incubated with LA in the presence of ${ }^{2} \mathrm{H}_{2} \mathrm{O}$, one ${ }^{2} \mathrm{H}$ was incorporated in the product $(9 Z, 11 E)$-CLA in pro- $R$ stereoconfiguration, hinting towards a reaction mechanism involving a prototropic shift (Kepler et al. 1970, Kepler et al. 1971). Moreover, the percentage of deuterium incorporated at carbon 13 was the same (88\%) after $18 \%$ as after $38 \%$ isomerization of the substrate had occurred. Based on these results, it was proposed that the isomerization involves the addition of a proton either directly from water or from some group in rapid equilibrium with water. Therefore, 
isomerization of LA was assumed to result either from the successive hydrationdehydration steps or from the direct addition and loss of a proton.

A hydration-dehydration mechanism would imply that (9Z)-12-hydroxy-octadecenoic acid (ricinoleic acid, RA) is an intermediate which is subsequently dehydrated to yield $(9 Z, 11 E)$-CLA. However, BFI did not produce CLA either from the naturally occurring $R$-RA nor a synthetically prepared racemic mixture, pointing towards an allylic shift mechanism coupled with protonation-deprotonation events.

Since the (9Z)-double bond of LA remains intact in $(9 Z, 11 E)$-CLA and proton addition at $\mathrm{C} 13$ was clearly demonstrated, the $\mathrm{C} 11$ and $\mathrm{C} 13$ atoms of LA were deduced as putative reaction centers. The kinetic isotope effect (KIE) due to the breaking of a C-H bond at $\mathrm{C} 11$ was demonstrated using 11-dideuterio-LA as BFI substrate, the deuteration did not alter the $\mathrm{K}_{\mathrm{m}}$ but had reduced the $\mathrm{V}_{\max }$ by about $60 \%$. Based on these results, Kepler et al. suggested concerted mechanism for the LA isomerization, involving the hydrogen removal from $\mathrm{C} 11$ and a stereospecific transfer ( $R$-configuration) of the solvent-derived proton by the enzyme to C13 of the substrate (Kepler et al. 1971).

Eukaryotic PUFA double bond isomerase from the red alga Ptilota filicina (PFI) was isolated and biochemically characterized by the group of Gerwick (Lopez \& Gerwick 1987, Wise et al. 1994, Wise et al. 1997, Zheng et al. 2002). In contrast to BFI and PAI (see 1.4), it shows a clear preference towards the $\omega-3$ long chain PUFAs, the best substrates are eicosapentaenoic acid $\left(20: 5^{\triangle 5 Z, 8 Z, 11 Z, 14 Z, 17 Z}\right.$, EPA) and docosahexaenoic acid (22: $6^{\Delta 5 Z, 8 Z, 11 Z, 14 Z, 17 Z, 21 Z}$, DHA), followed by AA and $\gamma$-LnA (Wise et al. 1994). Moreover, PFI was most active on the free FAs, therefore the carboxylate group was required for the FA binding. The distinct feature of PFI reaction is the formation of a conjugated triene, i.e. two double bonds of PUFA are shifted by the enzyme when three or four double bonds are present in the substrate. $\operatorname{LnA}$ and dihomo- $\gamma$ - $\operatorname{LnA}\left(20: 3^{\Delta 8 Z, 11 Z, 14 Z}\right)$ were transformed by PFI to the mixture of conjugated diene and triene, the latter product being predominant, while LA gave only conjugated diene PUFA, but the identity of this product was not established (Wise et al. 1997). The physiological role of conjugated PUFAs in marine algae is not well understood, however the mechanistical details of PFI reaction provided valuable analogies with bacterial PUFA double bond isomerases concerning the reaction mechanism. 

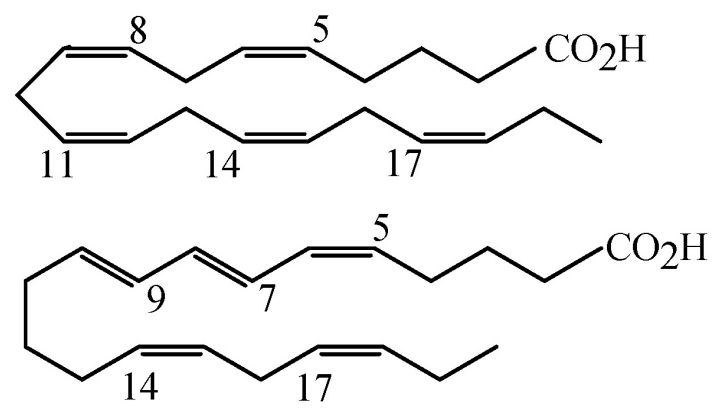

Fig. 3 Structures of EPA and conjugated triene product produced by PFI. The double bond positions are numbered. From (Wise et al. 1994).

Double bonds at positions $\triangle 5 Z, 8 Z, 11 Z$ in EPA and AA are converted by PFI to a conjugated system $\triangle 5 Z, 7 E, 9 E$, i.e. two double bonds at $\mathrm{C} 8$ and $\mathrm{C} 11$ are shifted towards the carboxy-terminus of FA (Fig. 3). This raises two questions related to the reaction route: which hydrogen atoms are abstracted by PFI in order to initiate isomerization and whether this allylic shift is a concerted step or conjugated diene serves as an intermediate. Incubation of PFI with $\mathrm{AA}$ in ${ }^{2} \mathrm{H}_{2} \mathrm{O}$ led to deuterium incorporation at position $\mathrm{C} 11$, while C12 was protonated with substrate-derived proton. When the enzyme was incubated in separate experiments with $(11 R)-,(11 S)$ - , $(8 R)$-, and $(8 S)$-deuterio- $\gamma$-LnA, PFI intramolecularly transferred the bis-allylic pro- $S$ hydrogen from the $\mathrm{C} 11$ position to the $\mathrm{C} 13$ position (corresponding to transfer from $\mathrm{C} 10$ to $\mathrm{C} 12$ in AA, Fig. 3). Furthermore, the bis-allylic pro- $R$ hydrogen at $\mathrm{C} 8$ in $\gamma$-LnA (the pro- $R$ hydrogen at $\mathrm{C} 7$ in AA, Fig. 3 ) was lost to solvent during the isomerization. With respect to the bis-allylic methylene groups, $\mathrm{C} 7$ and $\mathrm{C} 10$ of $\mathrm{AA}$ correspond to $\mathrm{C} 8$ and $\mathrm{C} 11$ of $\gamma$ - $\mathrm{LnA}$, thus the results can be transferred on C20 PUFAs. Furthermore, The formation of a diene by-product from dihomo- $\gamma$-LnA was suggested as the evidence that the overall reaction is likely not a concerted one (Wise et al. 1997).

PFI was not affected by known inhibitors of LOX, PGHS and cytochrome P450 enzymes and did not require molecular oxygen, excluding common oxidation-based mechanisms of PUFA formation (see below); neither EDTA nor o-phenanthroline showed a significant effect on PFI activity. Deglycosylation assays with highly purified 


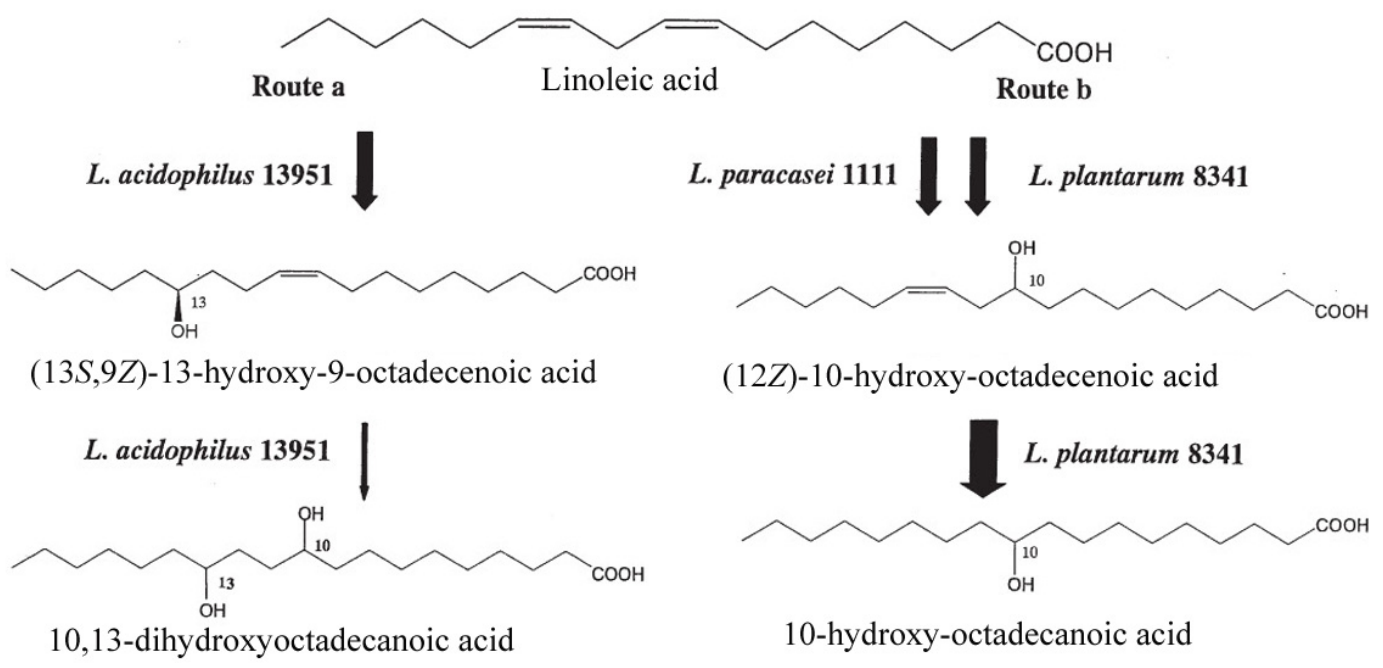

Fig. 4 Scheme of LA conversion to hydroxy FAs by Lactobacillus sp. From (Kishimoto et al. 2003).

enzyme demonstrated that native PFI from P. filicina is a glycoprotein (Zheng et al. 2002) The purified protein had a flavin-like UV spectrum and sequence analysis revealed the presence of a flavin-binding motif near the N-terminus (see also results, Fig. 13). These observations in connection with our results concerning PAI structure and reaction strongly suggest that PFI by the mechanism similar to that of PAI (see discussion for details).

A growing number of microbiological studies on PUFA transformations by bacteria are providing the evidence that a link between CLA production and formation of hydroxylated PUFA species exists in vivo. Several species of Lactobacillus were reported to accumulate CLA isomers along with (12Z)-10-hydroxy- and (12E)-10-hydroxyoctadecaenoic acid (10-HOE) during LA biotransformation under microaerobic conditions (Ogawa et al. 2001, Ogawa et al. 2005). The requirement for low oxygen concentration and suppression of culture growth by low levels of LA in the medium is similar to the conditions established for B. fibrisolvens. Feeding of isolated 10-HOE isomers to L. acidophilus cells resulted in declined levels of the substrate and production of CLA, however the interconversion of these molecules was not shown directly with isolated isomerase protein or by isotope labeling. In apparent contradiction, the production of hydroxy FAs by lactic acid bacteria without CLA accumulation was also 
reported (Kishimoto et al. 2003). L. acidophilus and L. plantarum strains transformed LA into 13-HOE, 10-HOE, 10,13-dihydroxy-octadecanoic acid (10,13-di-HO) and 10hydroxy-octadecanoic acid (10-HO). Based on this, two different pathways for hydroxy FA production were suggested (Fig. 4). The first one starts with the hydration of (12Z)double bond yielding 13-HOE and the further processing of the remaining (9Z)-double bond results in 10,13-di-HO. In the second pathway 10-HOE is initially formed and then reduced to $10-\mathrm{HO}$. It should be noted, that the origin of the hydroxy group in these products was not strictly confirmed, however based on other studies described below and the results of this work, it is clear that enzymatic water addition to the double bond occurs, rather than oxidation with molecular oxygen.

In a recent survey of human colon microflora thirty bacterial strains were studied in respect to their ability to metabolize LA (Devillard et al. 2007). The most active CLAsynthesizing strains were Propionibacterium freudenreichii subsp. Shermani forming a mixture of $(9 Z, 11 E)$-CLA, $(10 E, 12 Z)$-CLA and $(9 E, 11 E)$-CLA and Bifidobacterium breve forming a mixture of $(9 Z, 11 E)$-CLA and $(9 E, 11 E)$-CLA. In contrast, bacteria belonging to the Clostridium cluster, including Roseburia sp. and B. fibrisolvens, were producing a single substance, an uninidentified hydroxy FA and VA, respectively. Yet another thirteen strains were found to produce 10-HOE without CLA accumulation. The metabolic origin of hydroxy group in 10-HOE was established by incubating bacteria in the medium enriched in ${ }^{2} \mathrm{H}_{2} \mathrm{O}$, and GC-MS analysis revealed that indeed the $\Delta 9$-double bond of LA was hydrated during biotransformation. Based on these results Devillard et al. postulated that several pathways for LA metabolism by anaerobic gut bacteria exist: the observed CLA production may result either from direct action of a PUFA double bond isomerases or from the combined dehydration-isomerisation of 10-HOE, for which the enzymatic activity was not yet isolated (Fig. 5).

Some plants are able to produce conjugated PUFAs by a completely different mechanism compared to bacteria. Two so-called conjugases from Momordica charantia (MomoFadX) and Impatiens balsamina $(\operatorname{ImpFadX)}$ were isolated by Cahoon et al. (Cahoon et al. 1999). These enzymes are able to produce $\alpha$-eleostearic $\left(18: 3^{\triangle 9 Z, 11 E, 13 E}\right)$ and $\alpha$-parinaric $\left(18: 4^{\Delta 9 Z, 11 E, 13 E, 15 Z}\right)$ acids converting the (12Z)-double bond of LA and 


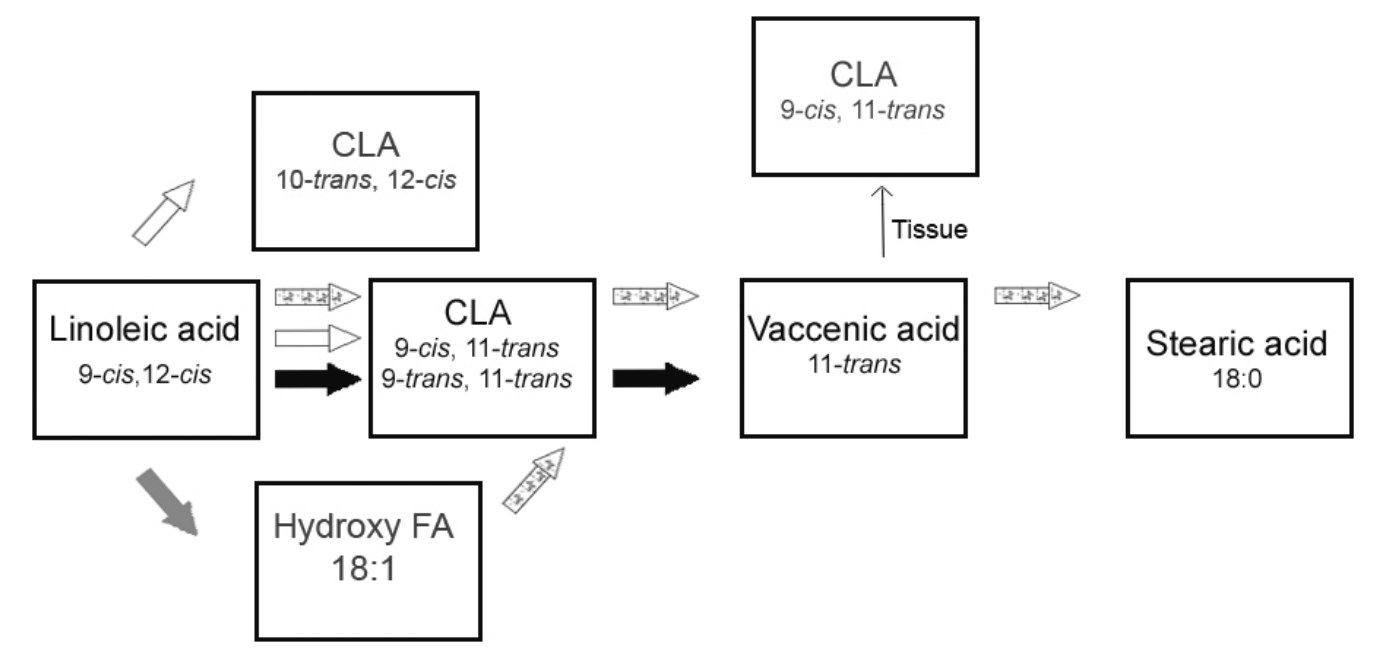

Fig. 5 Proposed routes of LA metabolism in anaerobic bacteria of human gut. From (Devillard et al. 2007).

Open arrows - activity of Lactobacillus, Propionibacterium, and Bifidobacterium species leading to the formation of CLA. Shaded arrows - activity of some Lactobacillus, Propionibacterium, and Bifidobacterium species and some Clostridium-like bacteria leading to the formation of hydroxy FA. Solid arrows -1 activity of Clostridium-like bacteria belonging to cluster XIVa leading to the formation of VA. Dotted arrows - activities observed in fecal microbiota, the responsible bacterial species are still unknown.

LnA into conjugated $(11 E, 13 E)$-system. Another divergent desaturase enzyme (FADX) from Aleurites fordii that modifies the (12Z)-double bond of LA, producing $\alpha$-eleostearic acid, was characterized (Dyer et al. 2002). The gene from Calendula officinalis, encoding for 8,11-LA desaturase transforming LA to calendic acid (CA, 18:3 ${ }^{\triangle 8 E, 10 E, 12 Z}$ ) was cloned (Cahoon et al. 2001, Fritsche et al. 1999), and its functional overexpression in Saccharomyces cerevisiae led to the accumulation of CA. The desaturase from Punica granatum, acting on (12Z)-double bond of LA and thus eliminating hydrogens from positions 11 and 14 was also described (Hornung et al. 2002, Iwabuchi et al. 2003). It was named PuFADX, and the recombinant protein produced by S. cerevisiae converted LA to punicic acid $\left(18: 3^{\triangle 9 Z, 11 E, 13 Z}\right)$. All these conjugases belong to the group of acyl-lipid desaturases containing catalytic diiron-oxo centers and catalyzing desaturation and hydroxylation of FAs. Depending on the enzyme, positional preferences vary between 
(9Z)- and (12Z)-double bonds in LA and LnA and formation of conjugated double bonds is achieved by stereo- and regiospecific hydrogen abstraction.

Despite the fact that the number of reports on CLA-producing activities in bacteria grows constantly, little is known about the identities of isomerase enzymes and the precise reaction mechanisms. BFI and eukaryotic PFI remain the best case studies up to date. A number of carbon-carbon double bond isomerases functioning in lipid metabolism pathways are known (for classification, see for example www.expasy.org/enzyme). Three dimensional structures of several enzymes were determined, providing insights in the diverse chemistry utilized for double bond activation; selected examples will be discussed in the next chapter.

\subsection{Comparative analysis of enzymatic mechanisms involved in carbon-carbon double bond isomerization and conjugated PUFA synthesis}

Apart from PUFA double bond isomerases, positional and geometrical isomerization of double bonds has been studied in several other enzyme systems (Fig. 6), notably in steroid delta isomerase (SDI), isopentenyl diphosphate (IPP):dimethylallyl diphosphate isomerase (IDI) type 1 and 2, 4-hydroxybutyryl-CoA dehydratase (4-BUDH)(Martins et al. 2004), trans-2-decenoyl-[acyl-carrier-protein] isomerase (FabA), $\Delta 3$-cis, $\Delta 2$-transenoyl-CoA isomerase (ECI), $\Delta 3,5-\Delta 2,4$-dienoyl CoA isomerase (DECI) and cis-trans FA isomerase (CTI) from Pseudomonas sp. Isomerization-conjugation of double bonds in the cyclic prostaglandin core by prostaglandin A (PGA) isomerase from human and rabbit serum was reported (Polet \& Levine 1975). Hydroperoxide lyase from the moss Physcomitrella patens was shown to form volatile compound (3Z)-nonenal, which was rapidly isomerized to $(2 E)$-nonenal, when 12-hydroperoxy-arachidonic acid was used as the substrate (Stumpe et al. 2006). No further biochemical data are available to date about the mechanisms of two latter enzymes. A broad class of iron-dependent oxygenases performs oxidation of PUFAs by molecular oxygen, coupled with double bond isomerization-conjugation, a well-studied members of this group are LOX and PGHS enzymes (Feussner \& Wasternack 2002, Kiefer et al. 2000, Malkowski et al. 2000, Schneider et al. 2007). Although oxygenase reaction involves redox changes and 


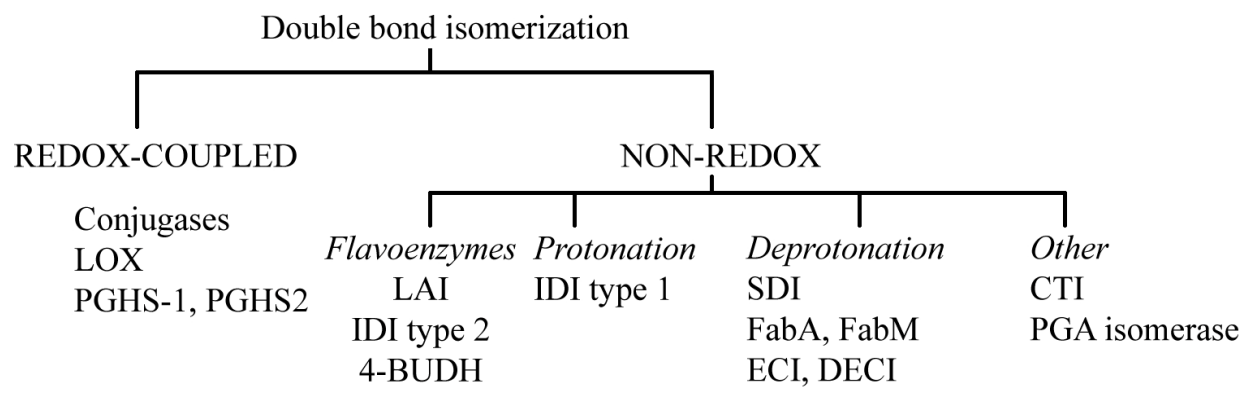

Fig. 6 Schematic overview of enzymatic mechanisms for carbon-carbon double bond isomerization. Enzymatic reaction are clustered according to net redox change as redox and non-redox. In the nonredox gropup the mechanism of substrate activation is given in italics. Abbreviations: LOX lypixygenase, PGHS - prostanglandine endoperoxide H synthase, LAI - LA isomerase, IDI isopentenyl diphosphate:dimethylallyl diphosphate isomerase, 4-BUDH - 4-hydroxybutyryl-CoA dehydratase, SDI - steroid delta isomerase, FabA, FabM - trans-2-decenoyl-[acyl-carrier-protein] isomerase, ECI - $\Delta 3$-cis, $\Delta 2$-trans-enoyl-CoA isomerase, DECI - $\Delta 3,5-\Delta 2,4$-dienoyl CoA isomerase, CTI - cis-trans isomerase from Pseudomonas sp., PGA - prostaglandin A.

therefore fundamentally differs from PUFA double bond isomerase activity, the mechanisms of substrate activation and the intermediate species are often similar for both pathways and will be discussed below. From the chemical point of view all listed enzymes fall into two broad classes, namely oxidoreductases, removing electrons from the PUFA substrate and transferring them to the oxygen or redox cofactor, and isomerases, catalyzing reactions with no redox change (Fig. 6). The isomerases in Fig. 6 act on an aliphatic chain and generally initiate structural rearrangements by hydrogen abstraction resulting in a reactive carbanion or radical. IDI-1 and CTI are exceptions and represent unique examples of double bond activation.

IDI-1 is involved in the isoprenoid biosynthesis, which proceeds by two pathways: the classical mevalonate pathway (Bach 1995) and the more recently identified methylerythritol phosphate (MEP) pathway (Rohdich et al. 2001). An important difference between both pathways is that the mevalonate pathway exclusively leads to IPP that must be isomerized to DMAPP by IDI-1 or IDI-2, whereas the MEP pathway branches in its final steps and results in the independent formation of IPP and DMAPP. 


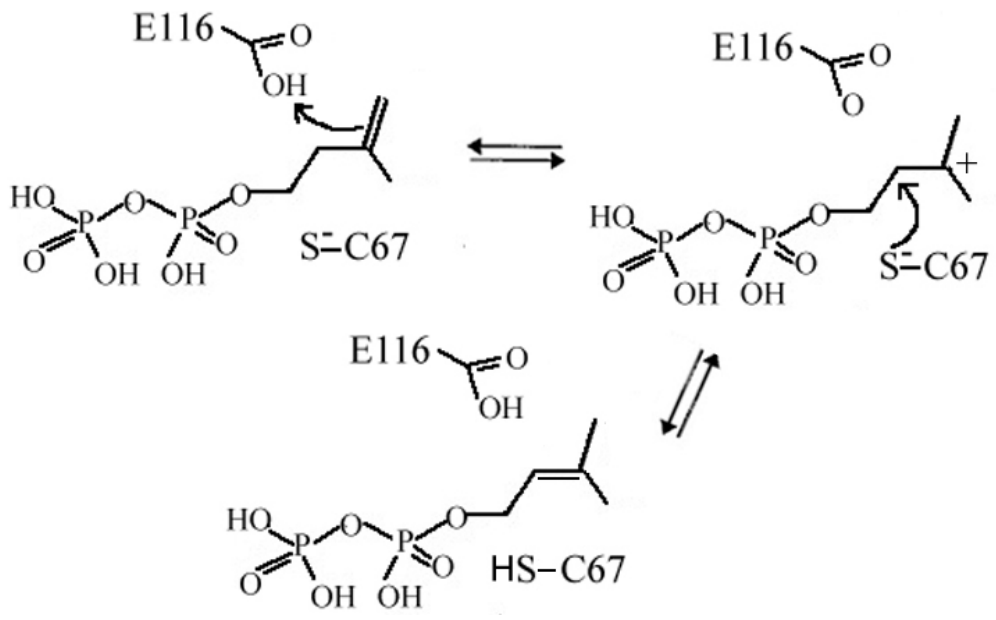

Fig. 7 Reaction mechanism of IDI type 1. From (Wouters et al. 2003).

The residues participatin in the reaction are labeled in one-letter code. The pyrophosphate goup of the substrate IPP is coordinated in the active site with divalent metal cation (not shown). After proton addition, most likely from Glu116, the tertiary carbcation is formed. Rearrangement of the carbcation is assisted by Cys67, which abstracts proton from C2 position of IPP, giving DMAPP.

The structures of human (Zheng et al. 2007) and E. coli IDI-1 in complex with substrate analogs (Wouters et al. 2003) are known. IDI-1 requires a divalent metal in order to bindthe phosphate moiety of IPP and to fold correctly. Protonation of the double bond of IPP, presumably by Glu116, initiates the reaction, leading to a tertiary carbcation (Fig. 7). This tertiary carbocation is stabilized by the metal-activated carboxylate form of Glu1 16 and by a $\pi$-cation interaction with the indole side chain of Trp121. The thiol group of Cys67 removes the proton from the $\mathrm{C} 2$ position, completing the isomerization.

Pseudomonas and Vibrio sp. rely on the CTI activity during the adaptation to all membrane affecting stimuli such as organic solvents, osmotic stress, heavy metals, heat shock, and membrane-active antibiotics (Heipieper et al. 2003). The protein is located in the periplasm, it requires neither ATP nor any other cofactor such as $\mathrm{NAD}(\mathrm{P}) \mathrm{H}$ or glutathione, the FAs attached to phoshpholipids (PL) are its substrates and therefore CTI operates even in the absence of de novo synthesis of lipids. A heme-binding site similar 


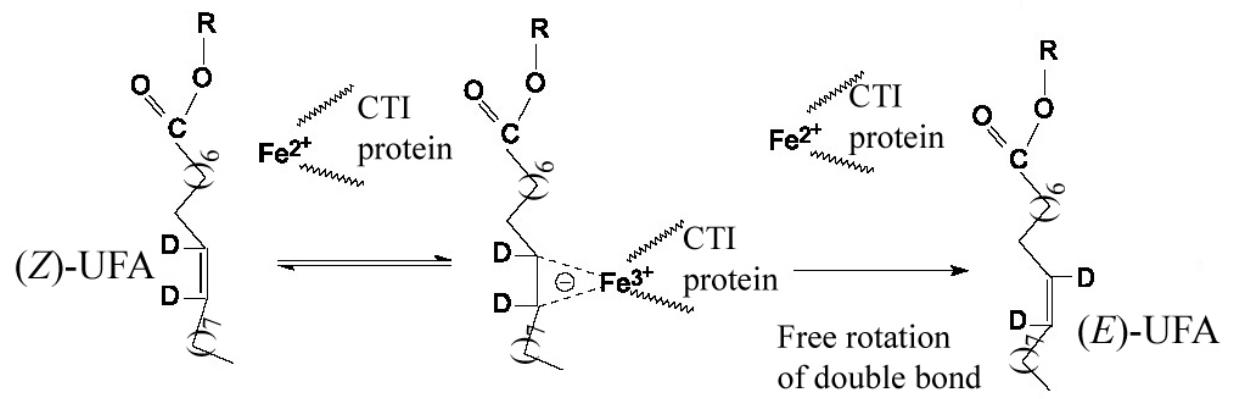

Fig. 8 Scheme of proposed CTI reaction mechanism. From (von Wallbrunn et al. 2003).

The heme iron in CTI active site coordinates (9Z)-double bond of OA, leading to decreased $\pi$-character of the latter and thus enabling free rotation around C9-C10 bond. In the experiments with double deuterated $\mathrm{OA}$ deuterium atoms at $\mathrm{C} 9$ and $\mathrm{C} 10$ position were retained after the CTI-catalyzed isomerization, in agreement with this model.

to cytochrome $\mathrm{c}$ was identified in the predicted CTI polypeptide. In the reaction with double deuterated OA it was demonstrated that CTI converts OA exclusively into double deuterated elaidic acid (EA, 18:1 ${ }^{\triangle 9 E}$ ) (von Wallbrunn et al. 2003). Thus, an enzymesubstrate complex in which the electrophilic heme iron (probably $\mathrm{Fe}^{3+}$ ) removes an electron from the (9Z)-double bond, was proposed as an intermediate (Fig. 8). The FA remains complexed with iron and due to the change of carbon hybridization from $\mathrm{sp}^{2}$ to $\mathrm{sp}^{3}$ the rotation around $\mathrm{C} 9-\mathrm{C} 10$ double bond is allowed. The double bond is then reconstituted after rotation to the $E$-configuration (Fig. 8). CTI seems to represent an unique enzymatic group since no significant sequence similarities were found between CTI and known proteins (von Wallbrunn et al. 2003).

A well-studied group of isomerases operates via elimination of an $\alpha$-proton adjacent to a keto-or ester-group; the resulting enolate is then protonated at the $\gamma$-position, resulting in a double bond shift. Acyl-CoA isomerases (ECI and DECI in Fig. 6) represent the classical example, essentially ECI shifts $(3 E)$ or $(3 Z)$ double bond in an acyl-CoA into ( $2 E$ ) position (Fig. 9). ECI plays an auxilary role in $\beta$-oxidation of PUFAs with double bonds at odd positions, for which the extra isomerization step is required as enoyl-CoA hydratase accepts only (2E)-acyl-CoA substrates. Structures of eukaryotic mitochondrial (Partanen et al. 2004) and peroxisomal (Mursula et al. 2001) isoforms of 


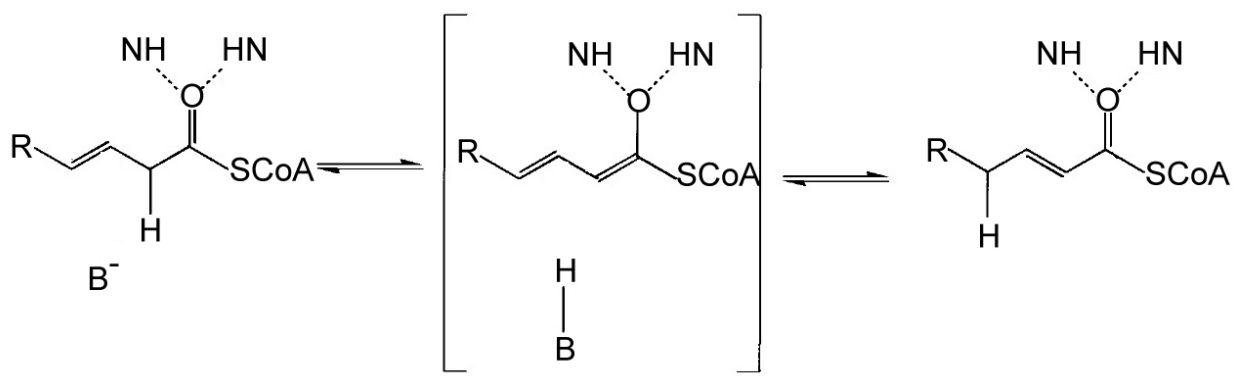

Fig. 9 Reaction mechanism of ECI. From (Mursula et al. 2001).

Acyl-CoA ester is shown as bound to the schematic active site. The FA tail can be of variable length. The two NH groups are peptide NH groups making an oxyanion hole. The catalytic base shown as B corresponds to Glu158 in yeast peroxysomal ECI. The reaction intermediate (shown in brackets) is stabilized by hydrogen-bonding interactions of the thioester oxygen atom in the oxyanion hole.

ECI were determined, as well as the structure of eukaryotic DECI, which processes substrates with conjugated double bonds (Zhang et al. 2001, Modis et al. 1998). Despite low sequence conservation between both ECI isoforms and DECI, all three proteins belong to the enoyl-CoA hydratase (crotonase) superfamily, and the conserved Glu residue was in every case shown to be the catalytic base abstracting the $\alpha$-proton in acylCoA. The intermediate dienolate is bound in an oxoanion hole, formed by two main chain amide bonds, thus the negative charge on the carbonyl oxygen is stabilized (Fig. 9). A single Glu residue is the catalytic base facilitating both deprotonation and reprotonation of the substrate in ECI isoforms. In contrast, it was suggested that in rat DECI Glu196 acts as a proton acceptor, but dienolate reprotonation is accomplished by Asp204 (Zhang et al. 2001).

Mechanistically similar to ECI, bacterial KSI enzymes display the fold of NTF2-like superfamily (Kim et al. 1997, Cho et al. 1998) and convert 5-androstene-3,17-dione to 4androstene-3,17-dione (Fig. 10). The reaction mechanism is essentially similar to ECI, with Asp40 being the catalytic base and reprotonating the dienolate intermediate, whereas the carbonyl oxygen of the latter is stabilized by hydrogen bonds to Tyr16 and Asp103. In an elegant structural study by Kraut et al. (Kraut et al. 2006) it was demonstrated that such electrostatic interactions in the oxoanione hole bring only about 300 fold increase to 


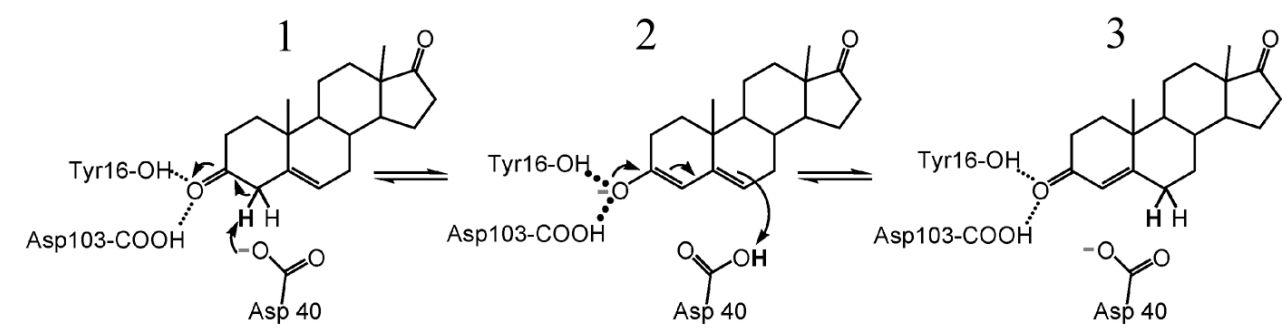

Fig. 10 Reaction mechanism of KSI. From (Kraut et al. 2006).

KSI converts 5-androstene-3,17-dione (1) to 4-androstene-3,17-dione (3). In the first step a general base, Asp40, removes a proton from the steroid to form a dienolate intermediate (2) (via a dienolatelike transition state), which receives hydrogen bonds from an oxyanion hole consisting of Tyr16 and protonated Asp103. In the second reaction step the steroid is reprotonated at a different position to give the product.

the catalytic efficiency of KSI. Therefore, it was concluded that a combination of both geometrical complementarity and electrostatic component likely accounts for the efficiency of enzymatic reaction compared to chemical isomerization.

Bacterial $\beta$-hydroxydecanoyl-ACP dehydratase (FabA protein in E. coli) catalyzes both the dehydration of $\beta$-hydroxydecanoyl-ACP and the isomerization of $(\Delta 2 E)$ decenoyl-ACP to (3Z)-decenoyl-ACP as the essential step in unsaturated FA (UFA) biosynthesis (Brock et al. 1967, Norris et al. 1964). A second dehydratase, called FabZ, was found in E. coli and the overlap in function between FabA and FabZ was shown (Heath \& Rock 1996). Therefore, Heath \& Rock postulated the roles of two dehydratases as following. Both FabA and FabZ function interchangeably in the cycles of FA elongation, but only FabZ can dehydrate unsaturated intermediates leading to UFAs. Unlike FabZ, bifunctional FabA protein is able to convert (2E)-decenoyl-ACP to (3Z)decenoyl-ACP, although this isomerase activity is much lower compared to dehydratase reaction of FabA. FabB ( $\beta$-ketoacyl-ACP synthase I) efficiently elongates (3Z)-decenoylACP, thus diverting the flow of intermediates into the UFA branch of FA synthesis. This enzyme is essential for UFA biosynthesis in E. coli. The crystal structures of bacterial FabA (Leesong et al. 1996) and FabZ (Kimber et al. 2004) were solved and both proteins display $\alpha+\beta$ "hot-dog" fold characteristic for thioesterase/thiol ester dehydrase- 
FabA

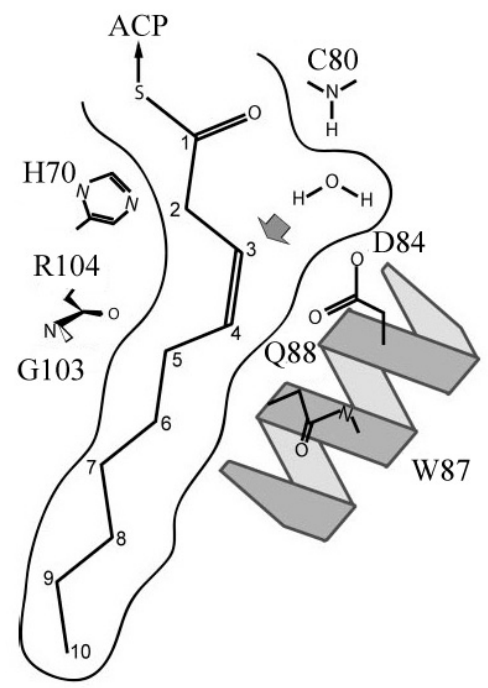

FabZ

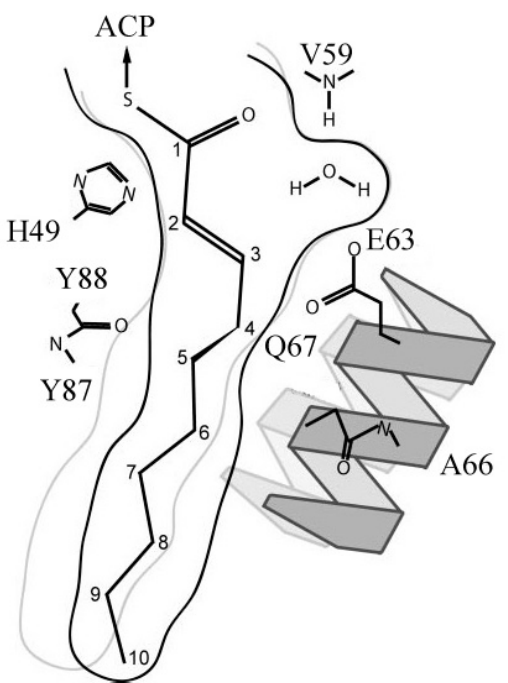

Fig. 11 Analysis of active site geometry in FabA and FabZ. From (Kimber et al. 2004).

FabA is depicted with a (3Z)-acyl chain bound and FabZ with a (2E)-acyl chain. The catalytic His residue is conserved in both enzymes, the protonation of leaving water molecule is assisted by acidic Asp84 and Glu63 residues. Low isomerase activity in FabA, but not not in FabZ, is explained by different shapes of substrate binding tunnels, as seen on the right panel (the trace of FabA pocket is shown as gray line)

isomerase family. Surprisingly, the structures overlay with rmsd of $1.4 \AA$ over 146 amino acid residues (Kimber et al. 2004). In contrast to Glu and Asp serving as a general base for $\alpha$-proton abstraction in ECI and KSI, an unprotonated His residue performs this function in FabA and FabZ. Asp84 and Glu63 (in FabA and FabZ, respectively) donate the proton to the leaving $\beta$-hydroxy group and the water molecule seen in the active site was suggested to result from the dehydratase reaction (Fig. 11). In FabA Asp84 could further deprotonate (2E)-decenoyl-ACP at $\gamma$-position, yielding after isomerization (3Z)decenoyl-ACP; consistent with this dual role, the FabA Asp84Ala mutant showed a 2 order of magnitude lower activity in both reactions. Kimber et al. reasoned that the major factor conferring isomerase activity in FabA, but not in FabZ, is the slight difference in the substrate-binding tunnel geometries (Fig. 11). Unlike FabA, FabZ is incapable to accommodate (3Z)-decenoyl-ACP, and this spatial restriction prevents FabZ from isomerization of (2E)-decenoyl-ACP (Kimber et al. 2004). 


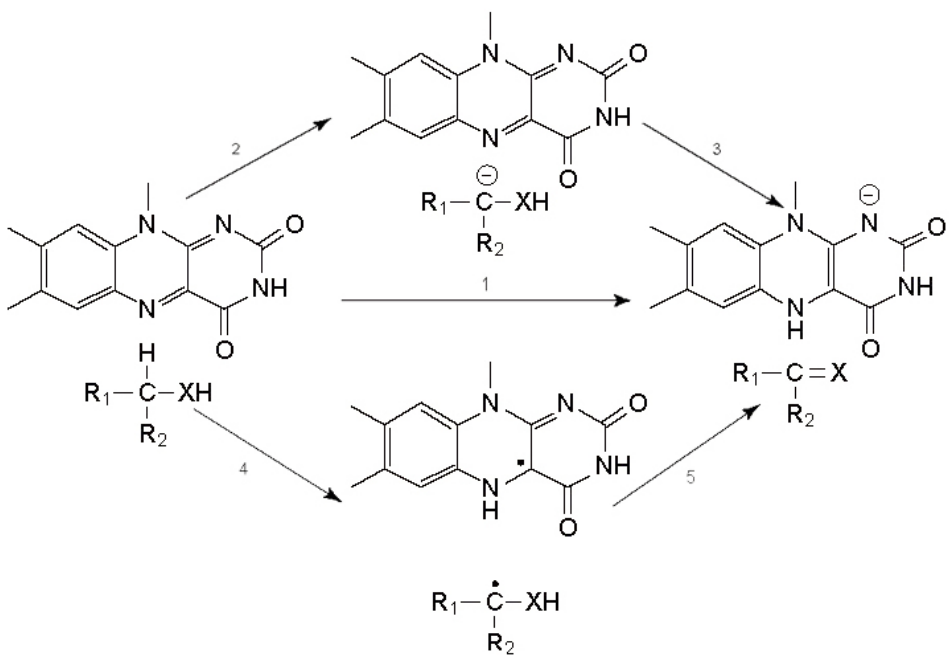

Fig. 12 Hydrogen abstraction by flavins. From (Fraaije \& Mattevi 2000).

The flavin redox states involved in direct hydride transfer (1), the carbanion mechanism $(2,3)$ and the radical mechanism $(4,5)$ reactions are shown. $\mathrm{R}_{1}$ and $\mathrm{R}_{2}$ indicate substituents of the $\mathrm{CH}$ atom undergoing oxidation, and $\mathrm{X}$ is the activating group.

Yet another isomerase involved in UFA biosynthesis in Streptococcus pneumoniae, FabM, was cloned and biochemically characterized (Marrakchi et al. 2002). It converts (2E)-decenoyl-ACP (formed by S. pneumoniea FabZ) to (3Z)-decenoyl-ACP and thus introduces a branching point from saturated FA to UFA one step downstream in the pathway, compared to E. coli. The amino acid sequence comparison revealed that FabM belongs to the hydratase-isomerase superfamily, along with ECI enzymes discussed above, which is not surprising taking into account that FabM essentially catalyzes the reverse reaction of ECI.

Another set of enzymes comprises 4-BUDH, IDI-2 and LA double bond isomerases (LAI), which all contain a flavin cofactor essential for the catalysis and therefore form mechanistically distinct group of double bond isomerases (Fig. 6). Although flavin presence in BFI and PFI was not directly confirmed, its involvement in the reaction is highly likely (see discussion for details), and therefore these proteins along with PAI fall into LAI group. Flavin adenine dinucleotide (FAD) and flavin mononucleotide (FMN) are ubiquitous redox cofactors employed by enzymes in two-electron as well as oneelectron transfers (Chapman 1999). This versatility arises from the unique ability of 
flavin isoalloxazine ring to cycle reversibly between fully reduced hydroquinone, half reduced semiqunone, and oxidized forms (Fig. 12).

IDI-2 converts IPP to DMAPP as shown in Fig. 7, but unlike IDI-1 both divalent metal cation and FMN are required for catalysis, in addition NADPH is essential for the activity (Kaneda et al. 2001). The 3D structure of IDI-2 from B. subtilis was solved, the enzyme was crystallized as an octamer, each subunit displaying classical TIM-barrel fold found in many flavoenzymes (Steinbacher et al. 2003). Even in the presence of the cofactor conserved regions implied in substrate binding were disordered and soaks with NADPH and IPP did not provide the enzyme-substrate complex structure. Later, the role of NADPH and FMN in the $S$. shibatae IDI-2 reaction was verified biochemically (Hemmi et al. 2004). Sub-equimolar amounts of NAPDH were required to reduce FMN and the enzyme was inhibited by a cofactor analog, 5-carba-5-deaza-FMN. The latter is unable to form the stable semiquinone radical, and therefore it was suggested that IDI-2 relies on a radical-based mechanism. The reaction is conceptually similar to that of IDI-1 (Fig. 7), but instead of the carbcation, an IPP radical is formed. The formation of FMN neutral semiquinone radical during IDI-2 reaction was recently confirmed by UV-VIS spectrometry coupled with redox potential measurements for the enzymes from Thermus thermofilus (Rothman et al. 2007) and Staphylococcus aureus (Kittleman et al. 2007). In addition, the substrate-dependent accumulation of the FMN radical was directly shown by electron paramagnetic resonance (EPR) spectroscopy (Kittleman et al. 2007).

Examples of dehydration and double bond shift based on $\alpha, \beta$-elimination catalyzed by general base were discussed above. Many anaerobic bacteria contain atypical dehydratases with unusual prosthetic groups such as $\mathrm{Fe}-\mathrm{S}$ clusters or flavins (Buckel \& Golding 2006). A well-studied example is 4-BUDH from Clostridium aminobutyricum, which catalyzes both the reversible oxygen-sensitive dehydration of 4-hydroxybutyryl$\mathrm{CoA}$ and the oxygen-insensitive isomerization of vinylacetyl-CoA (Fig. 13a) to crotonylCoA. The dehydration reaction requires the removal of an unactivated hydrogen atom from the $\mathrm{C} 3$ position of the substrate, which is mechanistically the most demanding step in the fermentation of $\gamma$-aminobutyrate to ammonia, acetate, and butyrate by $C$. aminobutyricum. 4-BUDH was crystallized as homotetramer with one [4Fe-4S]-cluster and one noncovalently bound FAD per 54-kDa subunit (Martins et al. 2004). 
(a)

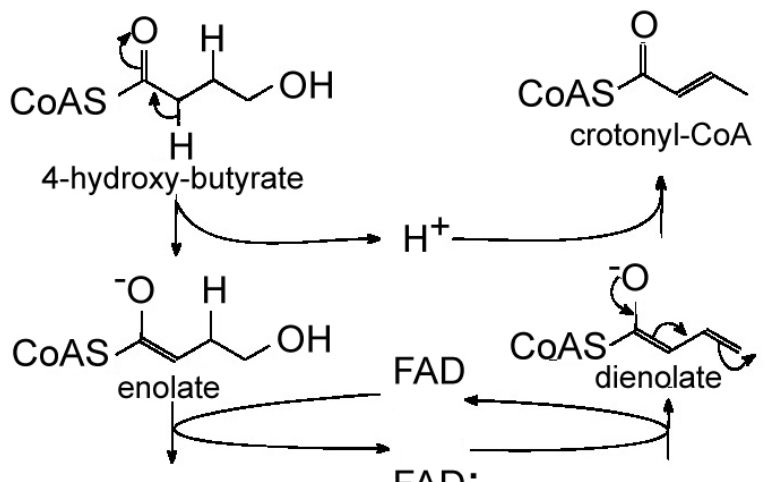

(b)
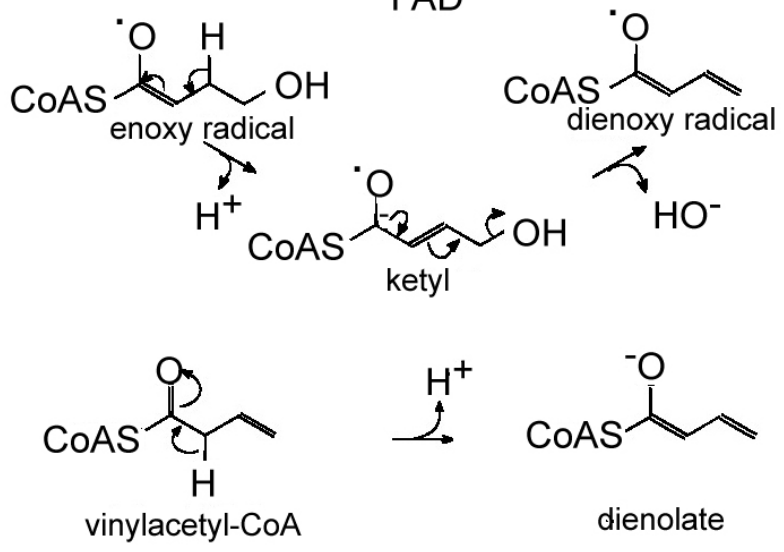

Fig. 13 Reaction mechanism of 4-BUDH. From (Muh et al. 1996).

(a) The catalytic cycle of 4-BUDH. The catalytic base His292 (not shown) abstracts the C2-proRhydrogen at the first step. FAD oxidizes the deprotonated enolate yielding enoxy radical and FAD semiquinone. The $\mathrm{C} 3$-proS-hydrogen is abstracted from the enoxy radical by the semiquinone anion and together with the hydroxy group forms the byproduct water molecule. The rearranged dienoxy radical is reduced by the semiquinone and resulting dienolate is re-protonated the $\mathrm{C} 4$ position by His292. (b) The isomerization of vinylacetyl-CoA requires only deprotonation at $\mathrm{C} 2$ and the redox part of the reaction is bypassed directly to dienolate.

The structure-derived dehydratase reaction mechanism based on substrate modeling in the active site of 4-BUDH and comparison of 4-BUDH and medium-chain acyl CoA dehydrogenase (MCAD) was suggested (Fig. 13b). First, C $\alpha$ of 4-hydroxybutyryl-CoA is deprotonated, presumably by His292 acting as a base. At the second step, FAD perfoms an one-electron oxidation of the enolate to form the enoxy radical and the flavin semiquinone anion. This anion acts as a base and abstracts the C3-pro $(S)$-hydrogen, forming the ketyl radical anion and the neutral semiquinone FADH. The ketyl radical 
anion then eliminates the hydroxyl group, which is polarized by the [4Fe-4S]-cluster acting as Lewis acid. Next, FAD semiquinone reduces the dienoxy radical to dienolate, with the recycling of FAD to the oxidized state. Final protonation at the $\mathrm{C} 4$ position of the dienolate by His-292 yields the product, crotonyl-CoA. Interestingly, the dehydratase activity is extremely sensitive to oxygen, while the isomerization of vinylacetyl-CoA to crotonyl-CoA (Fig. 13b) is a more stable reaction. An explanation seems to be the involvement of [ $4 \mathrm{Fe}-4 \mathrm{~S}]$-cluster only in the former reaction to assist water elimination (Martins et al. 2004).

LA isomerization differs from the reactions discussed so far, because the aliphatic carbon skeleton is rearranged without the assistance of activating carbonyl groups, thus a strong base is required to abstract hydrogen at the $\mathrm{C} 11$ position of LA. Both IDI-2 and 4BUDH eliminate hydrogen at an unactivated carbon by a radical mechanism, relying on flavin cofactor, but no direct evidence for the role of flavin in the catalysis was obtained so far for any LAI.

\subsection{Aim of the work}

Previous research in our group led to the cloning and preliminary characterization of several PUFA double bond isomerases. Among them, PAI was characterized as $(10 E, 12 Z)-C L A$ producing enzyme when expressed heterologously both in pro- and eukaryotic systems (Hornung et al. 2005). This work aimed to elucidate the mechanism of PUFA double isomerase reaction and for this the crystal structure of PAI and that of PAI-CLA complex was determined by X-ray crystallogaphy. FAD cofactor was discovered in the active site of PAI, leading to the working hypothesis that LA is transiently oxidized in the reaction course. Stereochemistry of the hydrogen transfer in LA was studied with isotopically labeled substrates and the role of FAD in the reaction was probed by a number of techniques. In addition, putative $(9 Z, 11 E)$-CLA-producing isomerases were cloned, overproduced and properties of purified proteins assayed with various PUFA substrates, the results of these studies hinted towards the role of hydroxylPUFAs in CLA formation. 


\section{Materials and methods}

\subsection{Chemicals}

All chemicals and solvents were purchased from Roth (Germany) or Sigma (Germany) unless stated otherwise; methanol, hexane, acetonitrile (all HPLC grade) were from Baker (USA); FAs were from Cayman Chemicals (USA) or Larodan (Sweden). Restriction enzymes and other DNA-modifying enzymes were from MBI Fermentas (Germany). Vectors for recombinant DNA and protein production were from Promega (USA), GE Healthcare (Sweden) and Novagen (USA). Basic molecular and biochemical techniques were performed as described in (Ausubel 1999).

\subsection{Recombinant DNA}

The cloning of PAI ORF into bacterial expression vector was described elsewhere (Hornung et al. 2005). Bifidobacterium breve isomerase (BBI), Lactobacillus reuteri isomerase (LRI), Streptococcus pyogenes hydratase (SPH) and PFI ORFs were amplified by PCR with primers listed in Suppl. Table 1. BBI, LRI, SPH and PFI ORFs were cloned from genomic DNA of respective organisms into pGEMT vector by Dr. E. Hornung (unpublished data).

PCR parameters were initial $94{ }^{\circ} \mathrm{C}$ denaturation for 2 minutes followed by 25 cycles of $94{ }^{\circ} \mathrm{C}$, denaturation for 30 seconds, $50{ }^{\circ} \mathrm{C}$ annealing for 30 seconds and $72{ }^{\circ} \mathrm{C}$ elongation for 1 minute. In the end a final $72{ }^{\circ} \mathrm{C}$ elongation phase of 5 minutes was added.

Resulting PCR products were purified with NucleoSpin Gel Extraction kit (Macherey-Nagel, Germany) and ligated into pGEMT vector using pGEMTeasy kit (Promega, Germany) according to manufacturer's instructions.

Positive clones were selected by colony-PCR with the same primers as for PCR amplification and plasmid DNA purified with NucleoSpin Plasmids kit (Macherey-Nagel, Germany). Resulting plasmid DNA was digested with the restriction enzymes listed in Suppl. Table 1 for each construct; empty vectors for protein overproduction were treated with the same enzymes.

Vector and insert were purified by agarose gel electrophoresis followed by NucleoSpin Gel Extraction kit (Macherey-Nagel, Germany). Insert was ligated with corresponding vector and ligation products were transformed into Escherichia coli XL1 
Blue cells (Stratagen, Germany). Positive clones were selected by colony-PCR with the same primers as for PCR amplification and plasmid DNA purified with NucleoSpin Plasmids kit (Macherey-Nagel, Germany). Insert presence was verified by restriction digestion with the enzymes listed in Suppl. Table 1. Finally, the identity of ORF was confirmed by sequencing with the BigDye Terminator v1.1 Cycle Sequencing Kit (Applied Biosystems, USA) according to the manufacturer's protocol; sequencing primers were as recommended by vector manufacturer.

\subsection{PCR based mutagenesis}

Site directed mutagenesis was performed using QuickChange kit from Stratagene according to manufacturer's instructions. Primers used to introduce mutations into PAI ORF are listed in Suppl. Table 2. Following mutations were created: Phe168 to Leu, Arg88 to Ala, Arg88 to Ser, Phe193 to Ala, double mutants Arg88 to Ser and Phe193 to Ala, and Phe168Gly169 to Gly168Phe169.

Silent mutations were introduced in the primers for screening purposes when possible. Template was pGEX6-PAI construct provided by Dr. E. Hornung. PCR program included initial $94{ }^{\circ} \mathrm{C}$ denaturation for $5 \mathrm{~min}$ followed by 18 cycles of $94{ }^{\circ} \mathrm{C}$ denaturation for $30 \mathrm{sec}, 50{ }^{\circ} \mathrm{C}$ annealing for $30 \mathrm{sec}$ and $68^{\circ} \mathrm{C}$ elongation for $6 \mathrm{~min}$. For the removal of original plasmid, PCR reactions were incubated for 1 hour at $37^{\circ} \mathrm{C}$ with 10 units of Dpn1 restriction enzyme. Plasmid DNA so obtained was transformed into Escherichia coli XL1 Blue cells, positive clones were selected by colony-PCR followed by restriction digest with the respective enzymes (Suppl. Table 2) and plasmid DNA was purified and sequenced as in 2.2 .

\subsection{Bacterial growth}

Escherichia coli BL21 Star strain (Invitrogen, Germany) was used for protein overproduction. Bacteria were cultivated in 2xYT medium ( $30 \mathrm{~g}$ trypton, $15 \mathrm{~g}$ yeast extract, $5 \mathrm{~g} \mathrm{NaCl}$ per $1000 \mathrm{~mL} \mathrm{H}_{2} \mathrm{O}$ ) supplied with $100 \mu \mathrm{g} / \mathrm{ml}$ carbenicillin (pGEX plasmids) or $25 \mu \mathrm{g} / \mathrm{ml}$ kanamycin (pET plasmids) at $37^{\circ} \mathrm{C}$ until OD $600 \sim 0.6-0.8$. Then IPTG was added to $0.1 \mathrm{mM}$, the cells were shifted to $16{ }^{\circ} \mathrm{C}$ and harvested by centrifugation (10 minutes at $9100 \mathrm{~g}$ ) after $18 \mathrm{~h}$ induction time. Cells were resuspended in buffer A (0.1 M Tris/HCl, pH 8.0, 0.15 M NaCl, $1 \mathrm{mM}$ EDTA, $1 \mathrm{mM}$ DTT) in 1:3 v/v ratio, frozen in liquid nitrogen and stored at $-20^{\circ} \mathrm{C}$. 
BBI, PAI and LRI were expressed as fusions with N-terminal GST affinity tag containing a PreScission protease site. BBI, LRI and SPH were also expressed with N-or C-terminal 6xHis tag as well as without any affinity tags (Suppl. Table 1).

\subsection{Protein solubility analysis and activity test}

For protein overproduction analysis cell lysates were obtained by freezing/thawing bacterial pellets in buffer $\mathrm{A}$, incubation with $0.1 \mathrm{mg} / \mathrm{ml}$ lysozyme for $10-15$ minutes at room temperature, sonication at $4{ }^{\circ} \mathrm{C}$ (Branson 350 sonifier equipped with microtip, $2 \times 30$ seconds, $20 \%$ power, $50 \%$ duty cycle) and centrifugation for 5 minutes at $20000 \mathrm{~g}$. After centrifugation, the pellet and supernatant fractions were analyzed by SDS-PAGE (4.8\% stacking gel, $12 \%$ separating gel). For this $30 \mu 1$ of the sonicated samples was used. All samples were mixed with $5 \mu 1$ of loading buffer (Fermentas, Germany) and heated at $96{ }^{\circ} \mathrm{C}$ for 5 minutes. The gel was run at $5 \mathrm{~mA} / \mathrm{cm}$ height for 1 hour and afterwards stained with Coomassie G250.

For the activity test with crude bacterial extract $100 \mu 1$ of the supernatant after centrifugation was mixed with $900 \mu 1$ of buffer A (or buffers identical to buffer A but with different $\mathrm{pH}$ value), $10 \mu \mathrm{g}$ of FA added from the stock in ethanol, the reaction was incubated $1 \mathrm{~h}$ at room temperature and FAs were extracted as described in 2.11.

Activity test with purified proteins was performed in a similar way, typically, 10 to 50 $\mu \mathrm{g}$ of protein was added to reaction mixture and 1 to $10 \mu \mathrm{g}$ of substrate was used for GC or GC-MS assays. For HPLC analysis 200 to $300 \mu \mathrm{g}$ of substrate was used.

\subsection{Protein purification}

\subsubsection{Affinity chromatography on Glutathione Sepharose}

PAI was purified at $4{ }^{\circ} \mathrm{C}$ as GST-fusion containing a PreScission protease site. Cells were disrupted by sonication in buffer A $+1.5 \%$ N-lauroyl-sarcosine (NLS) and centrifuged $20 \mathrm{~min}$ at $70000 \mathrm{~g}$. The supernatant was adjusted to $2 \%$ Triton X-100 and GST-PAI was bound to GSH-Sepharose, washed with buffer B (100 mM Tris/HCl, pH = $7.5,150 \mathrm{mM} \mathrm{NaCl})$ and eluted with buffer $\mathrm{C}(100 \mathrm{mM}$ Tris/ $\mathrm{HCl}, \mathrm{pH}=7.5,150 \mathrm{mM}$ $\mathrm{NaCl}, 1 \mathrm{mM}$ EDTA, $2 \mathrm{mM}$ DTT, 0.01\% Triton X-100, $20 \mathrm{mM} \mathrm{GSH})$. The fusion protein was cleaved with PreScission protease during dialysis against buffer D (50 mM Tris/ $\mathrm{HCl}$, $\mathrm{pH}=7.5,30 \mathrm{mM} \mathrm{NaCl}, 1 \mathrm{mM}$ EDTA, 2 mM DTT). 
BBI and LRI were also purified by this procedure; however, it turned out to be unsuitable for preparation of significant quantities of protein.

\subsection{2 $\mathrm{Ni}^{2+}$ affinity chromatography}

To improve the protein yield, BBI and LRI were purified as $\mathrm{N}$ - and C-terminal fusions, respectively, with 6xHis tag. Also SPH was purified as N-terminal 6xHis fusion, all purifications were done at $4^{\circ} \mathrm{C}$. Cells were lysed by adding $0.1 \mathrm{mg} / \mathrm{ml}$ lysozyme and 1 $\mu \mathrm{g} / \mathrm{ml}$ DNAse I in buffer $\mathrm{B}+10 \mathrm{mM} \mathrm{MgCl}_{2}$ and centrifuged $20 \mathrm{~min}$ at $70000 \mathrm{~g}$. The supernatant was loaded on HisTrap column (GE Healthcare, Sweden), the column washed with buffer B, and the protein eluted with buffer B $+0.5 \mathrm{M}$ imidazol. Protein concentration was estimated spectrophotometrically using calculated $\varepsilon_{280}=116990$, 120710 and $104335 \mathrm{M}^{-1} \mathrm{~cm}^{-1}$ for BBI, LRI and SPH, respectively.

\subsubsection{Ion exchange chromatography on Source 30Q Sepharose}

After the cleavage with PreScission protease, PAI and GST were separated by anion exchange chromatography on Source 30Q column (GE Healthcare). Dialysed sample was loaded on the column equilibrated in the buffer $\mathrm{D}$ and linear $\mathrm{NaCl}$ gradient applied using buffer E (50 mM Tris/HCl, $\mathrm{pH}=7.5,1 \mathrm{M} \mathrm{NaCl}, 1 \mathrm{mM}$ EDTA, $2 \mathrm{mM}$ DTT). PAI was concentrated to $10 \mathrm{mg} / \mathrm{ml}$ in $20 \mathrm{mM}$ Hepes/ $\mathrm{NaOH}, \mathrm{pH}=7.5$ by ultrafiltration (Vivaspin, membrane cutoff $30 \mathrm{kDa}$ ). Protein concentration was estimated by absorption using a calculated $\varepsilon_{280}=74500 \mathrm{M}^{-1} \mathrm{~cm}^{-1}$.

BBI and SPH were purified in a similar way to remove minor contaminants left after affinity purification. Protein eluted from HisTrap column was dialyzed against buffer D before the second purification step. EDTA and DTT were omitted from buffers D and E for the reasons discussed later.

\subsubsection{SDS-PAGE analysis of protein fractions}

Protein fractions after each purification step were analyzed by SDS-PAGE as described in 2.5. For concentrated protein samples series of dilutions ranging from 1 $\mathrm{mg} / \mathrm{ml}$ to $10 \mathrm{mg} / \mathrm{ml}$ was loaded on the gel to estimate proteolytic degradation (low protein amounts) and amount of contaminant proteins (high protein amounts) before crystallization screening. 


\subsection{Immunoblotting}

$10 \mu \mathrm{g}$ of proteins were subjected to SDS-PAGE as described in 2.5. Proteins were transferred to nitrocellulose membrane at $60 \mathrm{~V}, 4 \mathrm{~mA} / \mathrm{cm}^{2}$ gel surface for $1 \mathrm{~h}$. The membrane was washed with buffer B and blocked in buffer $\mathrm{B}+5 \%$ milk powder. Histagged proteins were detected using anti-His tag antibody (Sigma, Germany) in combination with a secondary antibody coupled with alkaline phosphatase and nitrotetrazolium blue/5-bromo-4-chloro-3-indolylphosphate staining.

\subsection{Spectroscopic assays}

Solutions of purified PAI, BBI and SPH all had yellow color, hinting towards the presence of some cofactor(s). For initial characterization UV-Vis spectra of purified proteins in the range 300-600 $\mathrm{nm}$ were recorded with singlebeam absorption spectrometer (Ultrospec 2100 pro, GE Healthcare). As reference spectra of FAD and FMN were recorded in the same way.

For these measurements BBI, LRI and PAI as well as FAD and FMN were brought to concentration $10-40 \mu \mathrm{M}$ in $50 \mathrm{mM}$ Tris/ $\mathrm{HCl}, \mathrm{pH}=7.5$. Fluorescence emission spectra in the range $460-650 \mathrm{~nm}(10 \mathrm{~nm}$ bandwidth) were measured at $298 \mathrm{~K}$ on a FluoroMax3 spectrofluorimeter at $2 \mathrm{mM}(\mathrm{FAD})$ and $6 \mathrm{mM}$ (PAI) concentrations in $40 \mathrm{mM}$ sodium phosphate $\mathrm{pH}=7.5$ after excitation at $450 \mathrm{~nm}(1 \mathrm{~nm}$ bandwidth $)$ and an integration time of $1 \mathrm{~s}$.

Non-covalent cofactor binding to BBI and SPH was confirmed in the following way. $200 \mu \mathrm{l}$ of the protein $(10 \mathrm{mg} / \mathrm{ml}$ in $50 \mathrm{mM}$ Tris/ $\mathrm{HCl}, \mathrm{pH}=7.5)$ was boiled for $10 \mathrm{~min}$ at $100{ }^{\circ} \mathrm{C}$ and centrifuged briefly. The spectrum of the supernatant was recorded in the same way as for native protein.

\subsection{Crystallization}

\subsubsection{Preliminary screening}

Crystallization screens Crystal Screen, Crystal Screen Lite and Crystal Screen 2 (Hampton research, USA); Footprint 1-3 and Structure Screen (Molecular Dimensions, USA) were used for initial crystallization trials. The reservoir volume was $400 \mu$, and the drop volume was $2 \mu \mathrm{l}(1 \mu \mathrm{l}$ of protein $+1 \mu \mathrm{l}$ of reservoir solution). The incubation of plates was performed at 10 and $20{ }^{\circ} \mathrm{C}$. PAI was at concentration $10 \mathrm{mg} / \mathrm{ml}$ in $20 \mathrm{mM}$ 
HEPES/NaOH, $\mathrm{pH}=7.5$. BBI and $\mathrm{SPH}$ were at 10 or $20 \mathrm{mg} / \mathrm{ml}$ in $0.1 \mathrm{M}$ Tris/ $\mathrm{HCl}, \mathrm{pH}=$ 7.5 .

\subsubsection{Optimized conditions for PAI crystallization}

Cubic-shaped crystals of PAI were grown at $10^{\circ} \mathrm{C}$ by sitting drop vapor diffusion from BisTris/HCl, $\mathrm{pH}=8.5,2 \mathrm{M} \mathrm{Li}_{2} \mathrm{SO}_{4}$ and either $2 \%$ PEG 400 or 2\% 1,4-butanediol. Plate-like crystals grew from $0.1 \mathrm{M} \mathrm{MES} / \mathrm{NaOH}, \mathrm{pH}=6.0,2 \mathrm{M}\left(\mathrm{NH}_{4}\right)_{2} \mathrm{SO}_{4}, 5 \% \mathrm{PEG}$ 400. The cubic crystals were directly cryocooled in liquid nitrogen while plates were cryoprotected in mother liquor supplemented with $12.5 \%$ PEG400. Crystals of the PAICLA complex were obtained by microseeding using cubic seeds obtained from mother liquor containing 1,4-butanediol to avoid competition with PEG400 and crystallization solutions containing $1 \mathrm{mM}$ LA. Crystals of the PAI-CLnA complex were grown from 0.1 M HEPES/NaOH, $\mathrm{pH}=7.5,2 \mathrm{M}\left(\mathrm{NH}_{4}\right)_{2} \mathrm{SO}_{4}, 0.8 \%$ 2-methyl-2,4-pentanediol. The platelike crystals were used for seeding in the presence of $1 \mathrm{mM} \mathrm{LnA.} \mathrm{FA} \mathrm{content} \mathrm{of}$ PAI-CLA and PAI-CLnA crystals was analyzed by GC and GC-MS as described below. Crystals were washed five times with $3 \mu \mathrm{l}$ of mother liquor, resuspended in $50 \mu \mathrm{l}$ of $\mathrm{H}_{2} \mathrm{O}$, and extracted with $100 \mu$ l of chlorophorm:methanol (1:1). The lower phase was dried and used for the analysis.

\subsection{Data collection, structure determination and refinement}

Data were collected in-house at $100 \mathrm{~K}$ on a MAR345 image plate detector-mounted on a MicroMax 007 generator and reduced with the HKL programs (HKL Research, Charlottesville, VA) (Table 1). The space group of the cubic crystals is $12_{1} 3$ with one molecule per asymmetric unit, Matthews coefficient $\mathrm{V}_{\mathrm{M}}=3.7$, and solvent content $65 \%$. The plate-like crystals are of space group $\mathrm{C} 2$ with one molecule in the asymmetric unit, Matthews coefficient $\mathrm{V}_{\mathrm{M}}=2.8$, and solvent content 55\%. The structure was determined by single isomorphous replacement with anomalous scattering of an iodide-soaked (mother liquor plus $0.25 \mathrm{M} \mathrm{KI}$ and 12.5\% PEG400) cubic crystal against data set native 1 (Table 1). A 13-atom substructure was found by SHELXD (Schneider \& Sheldrick 2002) at $3 \AA$ resolution. Phase extension to $1.86 \AA$ resolution and density modification was carried out in SHELXE (Sheldrick 2002). Automated model building with ARP/WARP (Lamzin \& Wilson 1993) placed 417 of 426 residues. COOT (Emsley \& Cowtan 2004) was used for manual model building. Refinement was performed with REFMAC5 (CCP4 
1994 ) with the same set of $5 \%$ of reflections reserved for $\mathrm{R}_{\text {free }}$ cross-validation (Brünger 1992) against data truncated at $1.95 \AA$ Å resolution. All other PAI structures were determined by molecular replacement using the first PAI structure as the starting model. Data collection and refinement statistics are summarized in Table 1. Structure quality was accessed with Procheck (Laskowski et al. 1993). Secondary structure was assigned with STRIDE (Frishman \& Argos 1995). Structure figures were created with BOBSCRIPT (Esnouf 1997) and rendered with RASTER3D (Merritt \& Murphy 1994) or PYMOL (www.pymol.org).

\subsection{GC and GC-MS analysis of lipids}

\subsubsection{Extraction and derivatization of lipids}

For lipid extraction $1 \mathrm{ml}$ of chlorophorm:methanol (1:1) and $50 \mu \mathrm{l}$ of $50 \%$ acetic acid were added to $1 \mathrm{ml}$ of reaction solution unless stated otherwise. After $2 \mathrm{~min}$ centrifugation at $15000 \mathrm{~g}$ the lower phase was transferred to the glass tube and dried by nitrogen stream (Bligh \& Dyer 1959).

To convert free FA to corresponding methyl esters, $400 \mu \mathrm{l}$ of methanol and $7 \mu \mathrm{l}$ of (trimethylsilyl)-diazomethane were added to the samples. After 15 min incubation at room temperature, remaining (trimethylsilyl)-diazomethane was inactivated by addition of $5 \mu \mathrm{l}$ of $5 \%$ acetic acid and samples were again dried under nitrogen stream. The FA methyl esters were dissolved in $100 \mu \mathrm{l}$ of acetonitrile, dried again and dissolved in $5 \mu \mathrm{l}$ of acetonitrile.

Esterified FAs were transesterified by adding $140 \mu \mathrm{l}$ of sodium methoxide $(0.1 \mathrm{M}$ solution in methanol) and $260 \mu \mathrm{l}$ of methanol/toluene $(2: 1, \mathrm{v} / \mathrm{v})$ solution to dried sample. Samples were incubated $30 \mathrm{~min}$ at room temperature, inactivated and dried as described above and dissolved in $5 \mu \mathrm{l}$ of acetonitrile.

For derivatization of hydroxy FA, dried samples were dissolved in $10 \mu \mathrm{l}$ of acetonitrile and $2 \mu \mathrm{l}$ of N,O-bis-(trimethylsilyl)-trifluoroacetamide (BSTFA) was added to each reaction to convert hydroxyl groups into trimethylsilylethers (TMS).

Linoleyl alcohol was incubated with PAI, products were extracted as above and analyzed without further derivatization.

For double bond position determination, FAs were converted to 5,5dimethyloxazoline (DMOX) derivatives. FA (200-500 $\mu \mathrm{g})$ was mixed in a glass vial tube 
with 2-amino-2-methyl-1-propanol $(0.25 \mathrm{ml})$. The vessel was flushed with nitrogen, stoppered, and placed overnight in a heating block at $190{ }^{\circ} \mathrm{C}$. After cooling the solid products were dissolved in $1 \mathrm{ml}$ of dichloromethane and transferred to a glass tube. $3 \mathrm{ml}$ of water and $3 \mathrm{ml}$ of hexane was added, mixed, and organic layer transferred to a fresh tube, dried and redissolved in chlorophorm. DMOX products were separated from unreacted components by thin-layer chromatography on silicagel plates (Merck, Germany). The solvent system was diethyl ether:petrol ether (2:1), the plate was sprayed by $0.3 \%$ solution of 8 -anilinonaphthalene-1-sulphonic acid, the DMOX bands scraped from the plate and extracted with water:hexane (1:1). The organic layer was dried, redissolved in acetonitrile and analyzed by GC-MS.

\subsubsection{GC and GC-MS analysis of lipids}

GC analysis was performed with an Agilent GC 6890 system (Waldbronn, Germany) coupled with a FID detector equipped with a capillary DB-23 column $(30 \mathrm{~m} \times 0.25 \mathrm{~mm}$; $0.25 \mu \mathrm{m}$ coating thickness; Agilent, Waldbronn, Germany). Helium was used as carrier gas $\left(30 \mathrm{~cm} \times \mathrm{s}^{-1}\right)$. The samples were measured with a split of 15:1 with an injector temperature of $220^{\circ} \mathrm{C}$. The temperature gradient was $150{ }^{\circ} \mathrm{C}$ for $1 \mathrm{~min}, 150-160{ }^{\circ} \mathrm{C}$ at 10 $\mathrm{K} / \mathrm{min}, 160-200{ }^{\circ} \mathrm{C}$ at $7 \mathrm{~K} / \mathrm{min}, 200-250{ }^{\circ} \mathrm{C}$ at $25 \mathrm{~K} / \mathrm{min}$ and $250{ }^{\circ} \mathrm{C}$ for $7 \mathrm{~min}$. CLA's were identified by authentic standards (Sigma, Germany).

GC-MS analysis was carried out using an Agilent 5973 Network mass selective detector connected to an Agilent 6890 gas chromatograph equipped with a capillary DB23 column ( $30 \mathrm{~m} \times 0.25 \mathrm{~mm}$; $0.25 \mu \mathrm{m}$ coating thickness; Agilent, Waldbronn, Germany). Helium was used as carrier gas $(1 \mathrm{ml} / \mathrm{min})$. The temperature gradient was $150{ }^{\circ} \mathrm{C}$ for 1 $\min , 150-160{ }^{\circ} \mathrm{C}$ at $10 \mathrm{~K} / \mathrm{min}, 160-200{ }^{\circ} \mathrm{C}$ at $7 \mathrm{~K} / \mathrm{min}, 200-250{ }^{\circ} \mathrm{C}$ at $25 \mathrm{~K} / \mathrm{min}$ and $250^{\circ} \mathrm{C}$ for $7 \mathrm{~min}$. Electron energy of $70 \mathrm{eV}$, ion source temperature of $230{ }^{\circ} \mathrm{C}$, and temperature of $260^{\circ} \mathrm{C}$ for the transfer line was used.

\subsection{HPLC analysis of FA}

HPLC analysis was carried out on an Agilent (Waldbronn, Germany) 1100 HPLC system equipped with a diode array detector. Absorption at $234 \mathrm{~nm}$ (conjugated diene system) and $210 \mathrm{~nm}$ (polyenoic FAs) were recorded simultaneously. Straight phase HPLC on a Zorbax RX-SIL column $(150 \times 2.1 \mathrm{~mm}, 5 \mu \mathrm{m}$ particle size; Agilent $)$ was used to separate the hydroxy FAs; the solvent system was $n$-hexane/2-propyl alcohol/acetic 
acid (100:1:0.1, by volume) at a flow rate of $0.2 \mathrm{ml} \mathrm{min}^{-1}$. FAs were extracted as in 2.11.1 and dissolved in $50 \mu \mathrm{l}$ mobile phase. The enantiomeric composition of the hydroxy FAs eluted from straight phase column was analyzed by chiral phase HPLC on a Chiralcel OD-H column $(150 \times 2.1 \mathrm{~mm}, 5 \mu \mathrm{m}$ particle size; Daicel Chemical Industries, Illkirch, France) with a solvent system of $n$-hexane/2-propyl alcohol/acetic acid (100:2:0.1, by volume) at a flow rate of $0.1 \mathrm{ml} \mathrm{min}^{-1}$. CLAs were analyzed on a silvermodified cation-exchange ligand covered silica sphere (Chromspher 5 Lipids, 250x4.5 mm, Varian, Darmstadt, Germany) with hexane/acetonitrile (100:0.2) as mobile phase and a flow rate of $1 \mathrm{ml} / \mathrm{min}$.

\subsection{Kinetic measurements}

All assays were performed in $100 \mathrm{mM}$ Tris/ $\mathrm{HCl}, \mathrm{pH}=7.5$ (Buffer F) at $25^{\circ} \mathrm{C}$. Fatty acid stock solutions were prepared in ethanol. For every measurement, $5 \mu$ of FA stock solution was mixed with buffer, 0.5 to $4 \mu \mathrm{g}$ of purified wt or mutant PAI protein was added and absorbance of solution at $234 \mathrm{~nm}$ was recorded with GE Healthcare Ultrospec 1100 pro spectrophotometer during $1 \mathrm{~min}$. Final reaction volume was $500 \mu 1$, thus ethanol concentration did not exceed $1 \%$. Concentration of CLA was calculated using $\varepsilon_{234}$ $=24000 \mathrm{M}^{-1} \mathrm{~cm}^{-1}$. For every point three independent measurements were performed, data averaged and fitted to Michaelis-Menten equation using Origin 6.1 software. Data were normalized to protein amount per reaction $(500 \mathrm{ng} / \mathrm{ml}$ or $10 \mathrm{nM})$.

\subsection{Cofactor exchange}

5-deaza-FAD was a generous gift of Prof. S. Ghisla. $200 \mu \mathrm{g}$ of purified PAI were diluted 10 fold in $6 \mathrm{M}$ guanidium chloride, $100 \mathrm{mM}$ Tris $\mathrm{pH}=7.5,10 \mathrm{mM}$ DTT. This solution was passed through $25 \mathrm{ml} \mathrm{G} 25$ desalting column (GE Healthcare) equilibrated in buffer F $+1 \mathrm{mM}$ DTT to remove FAD and to refold protein. Resulting protein solution was concentrated in Vivaspin concentrators, membrane cutoff $30 \mathrm{kDa}$ up to $0.2 \mathrm{mg} / \mathrm{ml}, 5$ fold molar excess of 5-deaza-5-carba-FAD was added, protein diluted 10 fold with Buffer $\mathrm{F}$ and concentrated again. The washing step was repeated twice; final protein concentration was about $1 \mathrm{mg} / \mathrm{ml}$ or $20 \mu \mathrm{M}$. With this preparation UV-Vis spectra were recorded and activity tests performed as described in 2.5. 


\subsection{Anaerobic photoreduction and redox potential measurement}

PAI was brought to $100 \mathrm{mM}$ Tris $\mathrm{pH}=7.0$ at concentration $20 \mu \mathrm{M}(1 \mathrm{mg} / \mathrm{ml}$ protein $)$. $2 \mu \mathrm{M}$ 5-deaza5-carba-FAD and $1 \mu \mathrm{M}$ of the following redox dyes were added: methyl viologen, 2-hydroxy-1,4-napthaquinone and phenazine methosulfate. Sample was transferred to anaerobic cuvette and made anaerobic by 20-30 cycles of vacuum and flushing with oxygen-free nitrogen. The cuvette was illuminated in the water bath at 25 ${ }^{\circ} \mathrm{C}$ for $5 \mathrm{~min}$ intervals with $300 \mathrm{~W}$ white lamp and allowed to equilibrate for $5 \mathrm{~min}$, UVVis spectrum recorded after each step. $10 \mathrm{mM}$ EDTA, $10 \mathrm{mM}$ sodium oxalate or just Tris were used as electron donors (Massey \& Hemmerich 1978).

\subsection{MALDI TOF analysis}

The purified SPH and BBI proteins were subjected to SDS-PAGE and further analyzed by in-gel digestion with trypsin and MALDI-TOF mass spectrometry in the Bioanalytical Mass Spectrometry division, Max-Planck Institute for Biophysical Chemistry, Goettingen. 
Table 1. Data collection, phasing and refinement statistics.

\begin{tabular}{|c|c|c|c|c|c|c|}
\hline Dataset & $\begin{array}{l}\text { 2B9W - native } \\
1 \text { (PEG400) }\end{array}$ & $\begin{array}{l}\text { 2B9X - KI soak } \\
\quad(\text { PEG400) }\end{array}$ & $\begin{array}{l}\text { 2BA9 - native } 2 \\
\text { (PEG400) }\end{array}$ & 2B9Y - apo & $\begin{array}{l}\text { 2BAB-CLA } \\
\text { complex }\end{array}$ & $\begin{array}{l}\text { 2BAC- }-\mathrm{LnA} \\
\text { complex }\end{array}$ \\
\hline Data collection & $50-1.95$ & $50-2.21$ & $50-1.95$ & $50-2.2$ & $50-2.0$ & $40-2.25$ \\
\hline Resolution range $(\AA)^{1}$ & $(2.00-1.95)$ & $(2.29-2.21)$ & $(2.02-1.95)$ & $(2.28-2.20)$ & $(2.07-2.00)$ & $(2.33-2.25)$ \\
\hline Space group & $\mathrm{I} 2{ }_{1} 3$ & $\mathrm{I} 2{ }_{1} 3$ & $\mathrm{C} 2$ & $\mathrm{I} 2{ }_{1} 3$ & $\mathrm{I} 2{ }_{1} 3$ & $\mathrm{C} 2$ \\
\hline Cell dimensions $\left(\AA,{ }^{\circ}\right)$ & 160.4 & 160.3 & $\begin{array}{c}\mathrm{a}=133.7 \\
\mathrm{~b}=60.8, \mathrm{c}=72.2 \\
\beta=115.8\end{array}$ & 160.4 & 161.8 & $\begin{array}{c}\mathrm{a}=133.7, \\
\mathrm{~b}=60.8, \mathrm{c}=71.8, \\
\beta=117.7\end{array}$ \\
\hline Unique reflections & $49143(2282)$ & $58536(1322)$ & 37527 (3804) & $32918(1695)$ & $46145(3465)$ & $20816(356)$ \\
\hline Multiplicity & $5.3(1.9)$ & $5.9(1.2)$ & $4.3(4.3)$ & $5.0(1.9)$ & $5.2(1.9)$ & $3.2(1.7)$ \\
\hline Completeness (\%) & $98.4(78.2)$ & $87.9(19.7)$ & $100(98.1)$ & $94.3(49.2)$ & $97.3(73.8)$ & $85.3(14.7)$ \\
\hline Mosaicity $\left(^{\circ}\right)$ & 0.49 & 0.85 & 0.65 & 0.96 & 0.57 & 1.05 \\
\hline $\mathrm{R}_{\mathrm{sym}}(\%)^{2}$ & $9.9(75.6)$ & $12.9(55.5)$ & $12.3(61.2)$ & $8.7(49.1)$ & $10.4(53.3)$ & $9.0(24.4)$ \\
\hline Average $I / \sigma(I)$ & $14.0(1.3)$ & $12.1(1.0)$ & $11.4(2.5)$ & $16.5(1.5)$ & $13.3(1.5)$ & $12.8(3.0)$ \\
\hline \multicolumn{7}{|l|}{ SIRAS Phasing } \\
\hline Resolution ( $\AA$ ) & & $20-4$ & & & & \\
\hline \# Iodide sites & & 13 & & & & \\
\hline FOM / CCmap & & $0.56 / 0.80$ & & & & \\
\hline Refinement & $35.87-1.95$ & $22.7-2.22$ & $46.4-1.95$ & $42.9-2.20$ & $27.0-2.00$ & $40.0-2.30$ \\
\hline Resolution range $(\AA)$ & $(2.00-1.95)$ & $(2.28-2.22)$ & $(2.00-1.95)$ & $(2.27-2.20)$ & $(2.05-2.00)$ & $(2.36-2.30)$ \\
\hline $\mathrm{R}_{\text {cryst }}(\%)^{3}$ & $16.4(28.0)$ & $17.7(27.1)$ & $21.3(27.3)$ & $15.6(25.2)$ & $16.3(26.9)$ & $15.8(18.6)$ \\
\hline
\end{tabular}




\begin{tabular}{|c|c|c|c|c|c|c|}
\hline $\mathrm{R}_{\text {free }}(\%)^{3}$ & $19.0(32.5)$ & $21.5(37.2)$ & $25.9(30.5)$ & $20.4(29.6)$ & $20.0(31.6)$ & $22.8(23.2)$ \\
\hline \# of residues / waters & $424 / 465$ & $423 / 354$ & $423 / 432$ & $423 / 511$ & $426 / 559$ & $424 / 467$ \\
\hline Coordinate error $(\AA)^{4}$ & 0.081 & 0.127 & 0.182 & 0.118 & 0.088 & 0.158 \\
\hline $\begin{array}{l}\text { rmsd Bonds / Angles } \\
\left(\AA,^{\circ}\right)\end{array}$ & $0.015 / 1.44$ & $0.010 / 1.21$ & $0.010 / 1.22$ & $0.013 / 1.34$ & $0.011 / 1.24$ & $0.011 / 1.31$ \\
\hline $\begin{array}{l}\text { Ramachandran plot } \\
(\%)^{5}\end{array}$ & $89.2 / 10.5 / 0.3$ & $87.3 / 12.2 / 0.5$ & $89.5 / 10.0 / 0.5$ & $87.9 / 11.9 / 0.3$ & $87.9 / 11.9 / 0.2$ & $89.2 / 10.5 / 0.3$ \\
\hline Average B values $\left(\AA^{2}\right)$ & $38.3 \pm 8.5$ & $48.6 \pm 7.2$ & $24.3 \pm 4.9$ & $35.5 \pm 8.0$ & $40.9 \pm 8.5$ & $27.3 \pm 6.3$ \\
\hline
\end{tabular}




\section{Results}

\subsection{PAI structure}

\subsubsection{PAI purification and biochemical properties}

PAI was overproduced in E. coli BL21 Star cells, and purified at $4{ }^{\circ} \mathrm{C}$ as a GSTfusion containing a PreScission protease site (Fig. 14a). Most of the protein was found in insoluble fraction after cell lysis, therefore high concentration of the strong ionic detergent NLS was added to the lysate before sonication. NLS was shown to increase the yield of GST-tagged proteins several fold (Frangioni \& Neel 1993). Upon concentration, it was noticed that PAI is yellow in color and this color persisted during dialysis or gelfiltration. The absorption spectrum of PAI in the range $300-500 \mathrm{~nm}$ shows two bands at 370 and $460 \mathrm{~nm}$ (Fig. 14b), typically present in the enzymes containing oxidized flavin. These spectral bands resulting from the absorbance of an isoalloxazine ring can be shifted by several nanometers compared to the spectrum of free FAD (Fig. 14b) or FMN (not shown). The spectrum of PAI and the fact that the cofactor remained in the solution after the protein was heat-precipitated, suggested the presence of a noncovalently bound oxidized FAD. The cofactor was also retained during crystallization (Fig. 14d, e).

Flavins possess strong fluorescence at $525 \mathrm{~nm}$ after excitation at $450 \mathrm{~nm}$ (Chapman 1999). Comparison of the emission spectra of FAD and PAI reveals typical flavin fluorescence of the latter (Fig. 14c). Very often proteins strongly quench fluorescence of bound flavin, which is also the case for PAI. The maximal fluorescence emission intensity of PAI was $>80$-fold lower than that of free FAD.

\subsubsection{Crystallization, data collection, structure determination, and refinement}

Initial screening provided two conditions where three-dimensional crystals of PAI grew at $10{ }^{\circ} \mathrm{C}$ after 3-5 days. Precipitant from Structure screen No. 18 contained $0.1 \mathrm{M}$ Tris/HCl, $\mathrm{pH}=8.5,2 \mathrm{M} \mathrm{Li}_{2} \mathrm{SO}_{4}, 2 \%$ PEG400 and gave yellow cubic-shaped PAI crystals (Fig. 14d). These crystals diffracted in some cases to less than $2 \AA$ (Fig. 14f) and one of such datasets was used for structure refinement with a resolution of $1.95 \AA$ (Table 1). Plate-like crystals (based on the condition Crystal Screen No. 39) grew from $0.1 \mathrm{M}$ $\mathrm{Mes} / \mathrm{NaOH} \mathrm{pH}=6.0,2 \mathrm{M}\left(\mathrm{NH}_{4}\right)_{2} \mathrm{SO}_{4}, 5 \%$ PEG400 (Fig. 14e), diffracted somewhat poorer (Fig. 14g) and the structure based on the best dataset was refined only to $2.2 \AA$ (Table 1). As turned out later, a PEG400 molecule was occupying the substrate-binding 
(a)

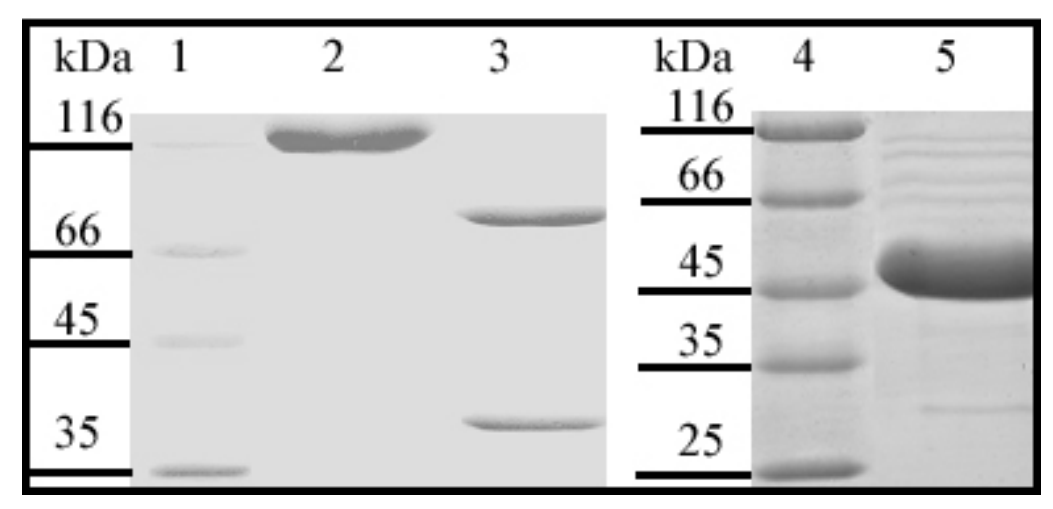

(b)

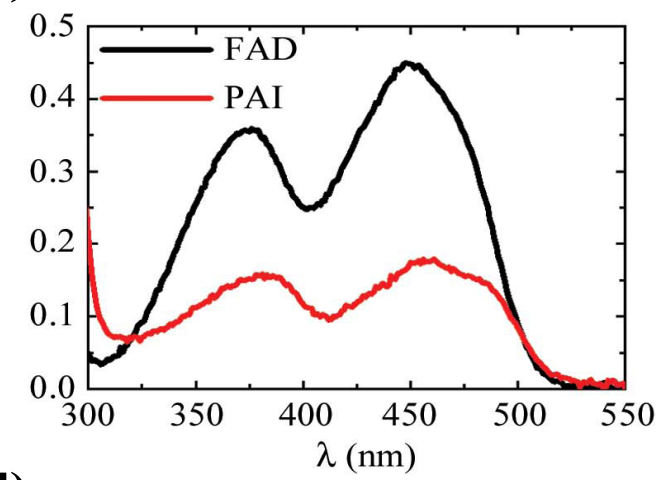

(d)

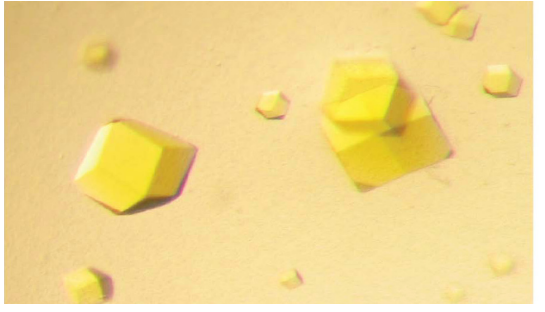

(f)

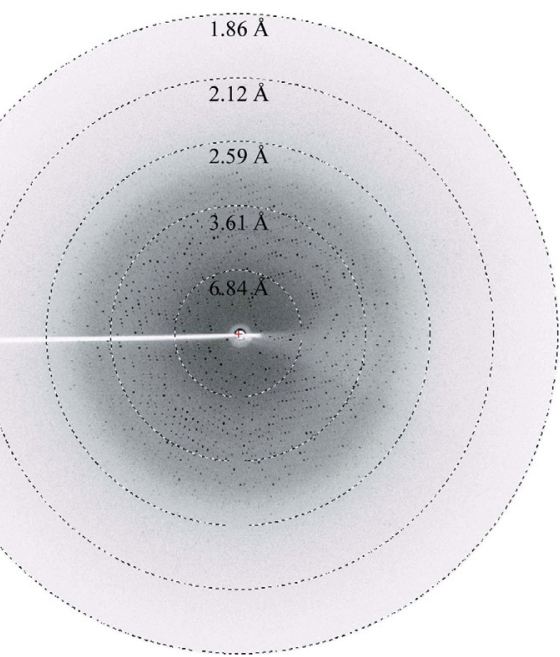

(c)

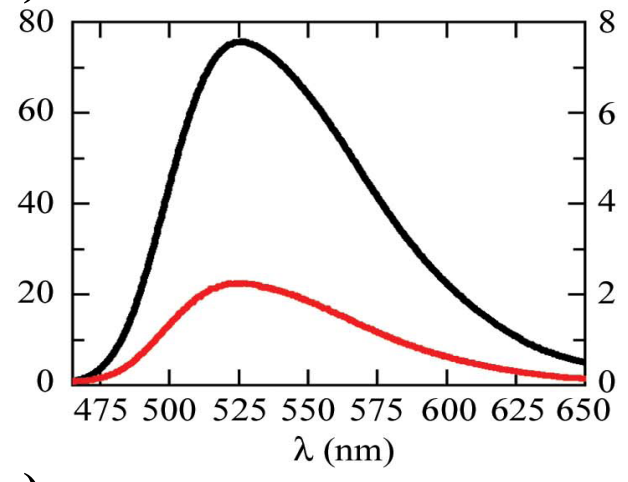

(e)

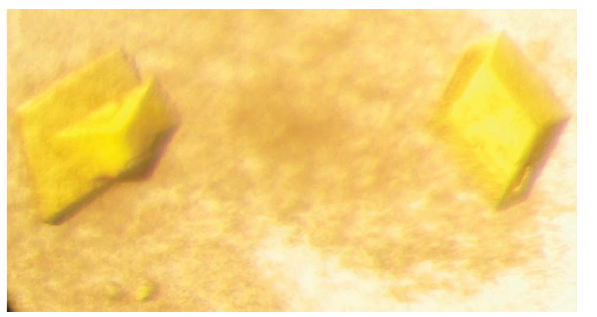

(g)

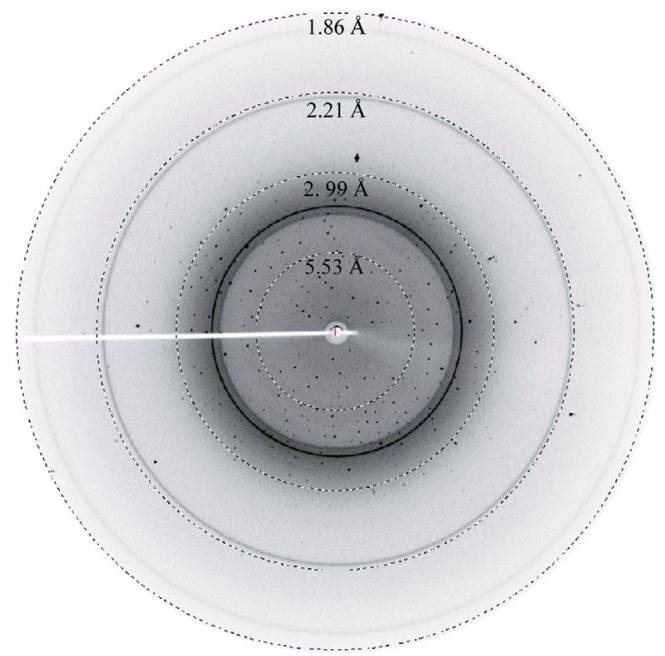


Fig. 14 PAI purification, spectral properties and crystallization.

(a) Purification of recombinant PAI. Lanes 1 and 4 - MW marker, 2 - PAI-GST fusion eluted from affinity resin, 3 - cleavage with PreScission protease, 5 - PAI purified by ion exchange chromatography. (b) UV spectra of $40 \mathrm{mM}$ solutions of free FAD (black curve) and PAI (red curve) show the two characteristic absorption maxima for an oxidized flavin (FAD or FMN) at 360-390 nm and 440-470 nm. (c) The fluorescence emission spectra of free FAD (2 mM, black curve) and PAI (6 $\mathrm{mM}$, red curve) show the characteristic flavin emission at $525 \mathrm{~nm}$. Cofactor fluorescence is strongly quenched when bound to the protein (compare the right ordinate). (d) Cubic PAI crystals from $0.1 \mathrm{M}$ BisTris/HCl pH = 8.5, $2 \mathrm{M} \mathrm{Li}_{2} \mathrm{SO}_{4}, 2 \%$ (v/v) PEG400. (e) monoclinic PAI crystals from $0.1 \mathrm{M}$ $\mathrm{MES} / \mathrm{NaOH} \mathrm{pH}=6.0,2 \mathrm{M}\left(\mathrm{NH}_{4}\right)_{2} \mathrm{SO}_{4}, 5 \%(\mathrm{v} / \mathrm{v})$ PEG400. The longest dimension in both crystal forms is $0.2 \mathrm{~mm}$. (f) and (g) show diffraction patterns of the cubic and monoclinic crystals, respectively, collected in-house on a MAR345 imaging-plate detector at a distance of $150 \mathrm{~mm}$ after $10 \mathrm{~min}$ exposure. The outer resolution is $1.86 \AA$.

channel, raising the need to modify the organic part of the precipitant solution in order to allow FA binding. Therefore the condition Structure Screen No. 16 was modified by replacing PEG400 with a range of organic solvents in the same concentration. The best results were obtained with 1,4-butanediol, which was used for co-crystallization of PAI with LA. Both vapor diffusion in the presence of FA and soaking of cubic crystals in mother liquor supplemented with FA resulted in FA binding judged by the change of crystal color from yellow to dark-orange (data not shown). However, the former method produced crystals of irregular shape and microseeding of PAI solution mixed with mother liquor containing $1 \mathrm{mM} \mathrm{LA}$ with cubic crystals obtained from 1,4-butanediol mother liquor was chosen to produce the PAI-CLA complex. The same procedure was used for PAI-LnA complex; there the best crystals grew from modified condition Crystal Screen No. 39 after seeding as described in 2.9.2.

Initial attempts to solve structure by SIRAS or MIR methods with heavy metal ions as source of anomalous signal failed due to instability of PAI crystals in the presence of $\mathrm{mM}$ concentrations of heavy metal salts. In the end, the structure of PAI was determined inhouse by the SIRAS method using iodide as the heavy atom. Halide soaking was described as potentially rapid method for either in-house (using iodide) or synchrotron (using bromide) phasing by SAD or SIRAS (Dauter et al. 2000). Crystals were briefly 
(a)

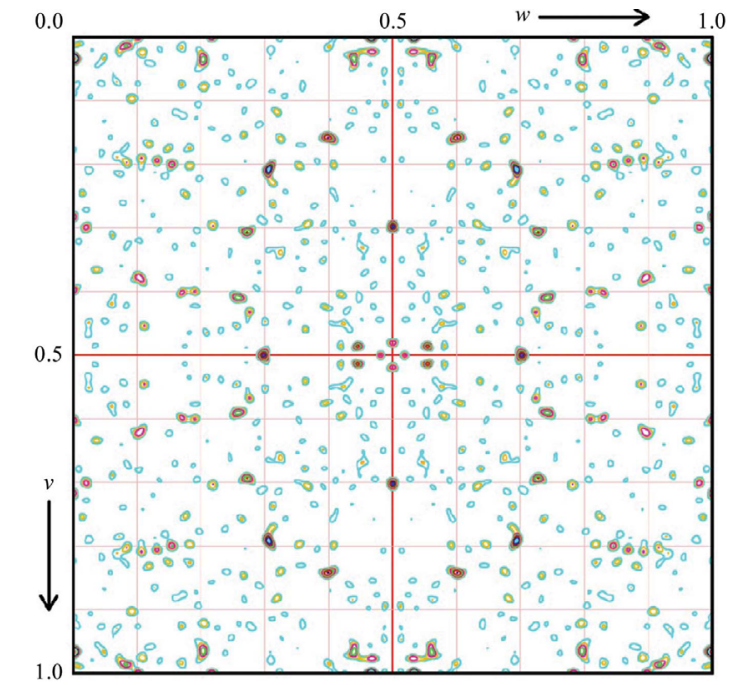

(b)

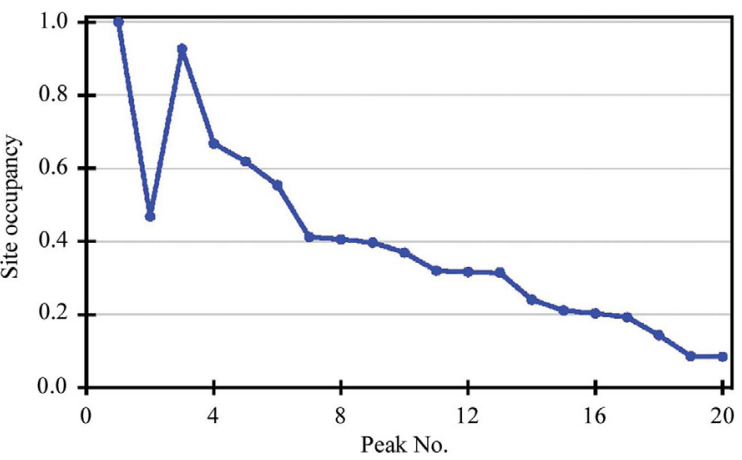

(c)

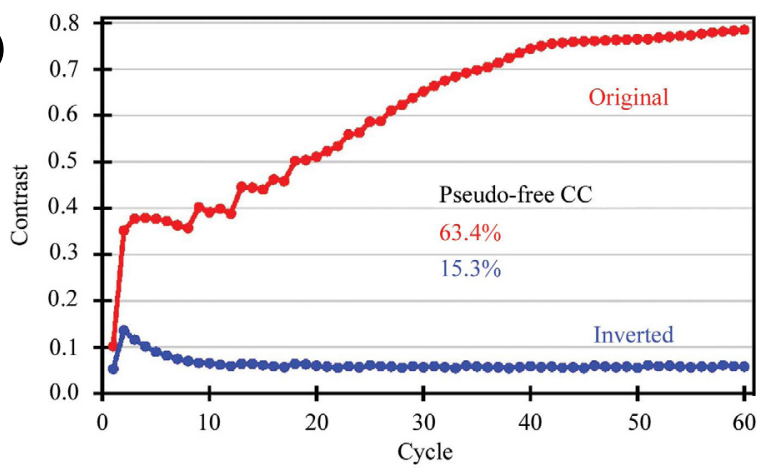

(d)

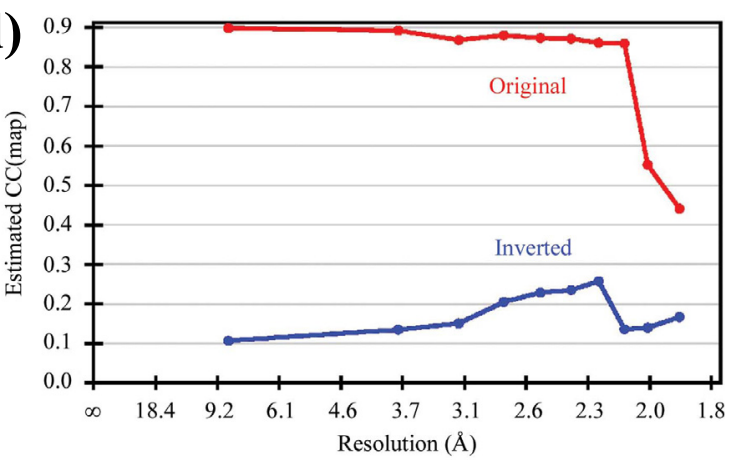

(e)

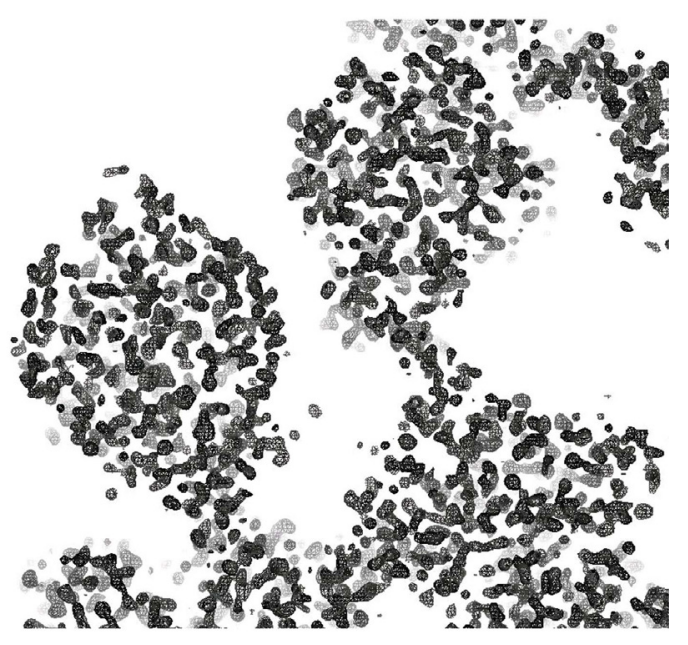


Fig. 15 SIRAS phasing of cubic PAI.

(a) Anomalous difference Patterson map at section $u=0.5$ calculated to a resolution of $3 \AA$ showing several distinct peaks for bound iodide. Contours are in steps of $1 \sigma$ and are coloured differently for each level. (b) Graphical representation of Suppl. Table 3 showing a drop in occupancy to $\sim 0.2$ at site No. 14. Iodide 2 is located on a crystallographic twofold. The top 13 sites were used as input for density modification and enantiomer selection in SHELXE. (c) Progress of density modification and phase extension monitored by the protein/solvent contrast versus modification cycle. While the correct hand of the substructure is immediately apparent, the quality of the electron density requires at least 60 cycles for convergence. (d) Estimated map correlation coefficient as a function of resolution calculated with density-modified phases. (b), (c) and (d) were generated with HKL2MAP (Pape 2004). (e) Experimental electron-density map contoured at $2.0 \sigma$ after density modification and phase extension in SHELXE. The $30 \mathrm{~A}^{\circ}$ section encompasses three molecules and shows a clear solvent boundary.

$(<1 \mathrm{~min})$ soaked in mother liquor supplemented with $1 \mathrm{M} \mathrm{KI}$, but tended to crack and failed to diffract X-rays. Typically, high concentrations of $>0.5 \mathrm{M}$ of the halide salt are used for soaking (Uson et al. 2003), as halides form only weak and non-covalent bonds to both ionic and hydrophobic areas of the protein surface. The highest KI concentration that still retained morphology and diffraction of cubic PAI crystals was $0.25 \mathrm{M}$ (Table 1), which might result in a reduced occupancy of the derivative sites.

Since the cubic crystal form of PAI had the high symmetry (I2 33$)$ and solvent content (65\%) density modification could be performed efficiently once a halide substructure has been found. After successful derivatization an anomalous difference Patterson map at Harker section $\mathrm{u}=0.5$ calculated to $3 \AA$ resolution with the origin removed showed a number of significant peaks $>10 \sigma$ (Fig. 15a). Initial heavy-atom substructure solution was obtained in a SIRAS experiment against data set native I (Table 1). The derived substructure of 13 sites with relative occupancy $>0.2$ had a CC of 0.51 and 0.39 for all/weak reflections, respectively (Fig. 15b and Suppl. Table 3). Density modification and phase extension using this substructure immediately established the correct hand of the substructure, but required at least 60 cycles to converge at the maximum protein/solvent contrast (Fig. 15c). Electron density calculated with these phases had an overall estimated map CC of 0.85 to $2.2 \AA$ resolution (Fig. 15d) and showed a clear solvent boundary (Fig. $15 \mathrm{e})$. 
Five more crystal structures of PAI in space groups $\mathrm{I}_{1} 3$ and $\mathrm{C} 2$, and either in the apoform or bound to products or PEG400, were subsequently determined by molecular replacement and refined to resolutions of $2.25 \AA$ or higher (Table 1). The maximum rmsd within these structures is $0.3 \AA$, independent of crystal lattice or complex state. Thus crystal-packing artifacts on the conformation of PAI can be excluded based on this high similarity between independent structures.

\subsubsection{Structure of PAI and PAI-CLA complex}

\subsubsection{Overall structure description of free PAI}

PAI comprises three intricately connected domains. Domain 1 (residues 1-77, 196275 , and 371-424) represents a mixed $\alpha / \beta$ FAD-binding domain, domain 2 (residues 77195 ) is predominantly $\alpha$-helical, and domain 3 (residues 276-370) is essentially fourstranded $\beta$-sheet interspersed with two $\alpha$-helices (Fig. 16a). Domain 1 closely resembles a variant of the Rossmann fold for dinucleotide binding found in the glutathione reductase family GR1 (Dym \& Eisenberg 2001), consisting of a central 4-stranded parallel $\beta$-sheet surrounded by two $\alpha$-helices. The conserved GxGxxG(x) $)_{18} \mathrm{E}$ motif of the GR1 family includes the P-loop and a glutamate residue that binds to the phosphate and ribose moieties of FAD, respectively. In PAI this motif is located within the first $37 \mathrm{~N}$-terminal residues. This region is highly conserved among GR1-flavoproteins and also contains several residues that engage in hydrophobic contacts with FAD (Fig. 17). Not surprisingly, the closest structural homologues of PAI identified by the DALI program (Frishman \& Argos 1995) are FAD-containing enzymes yeast polyamine oxidase (PAO) (Huang et al. 2005) and UDP-galactopyranose mutase (Sanders et al. 2001), with Zscores of 24.9 and 15.5, respectively. The closest non-flavin-containing structural homologue is guanine nucleotide dissociation inhibitor (GDI) with a DALI Z-score of 13.1 (Schalk et al. 1996). As GDI does not bind FAD, the reason for its close structural homology to flavoproteins is unclear.

PAI domains 2 and 3 have no structural homologues in DALI database but display a roughly similar organization as in UDP-galactopyranose mutase and PAO (PDB ID 1I8T and 1RSG, Fig. 16c), with high rmsd values of $12 \AA$ after superposition of all $\mathrm{C}_{\alpha}$ atoms. Between domains 2 and 3 a narrow C-shaped tunnel of ca. $30 \AA$ length stretches from the surface of PAI towards the isoalloxazine ring of FAD. To summarize, domain 1 binds to 

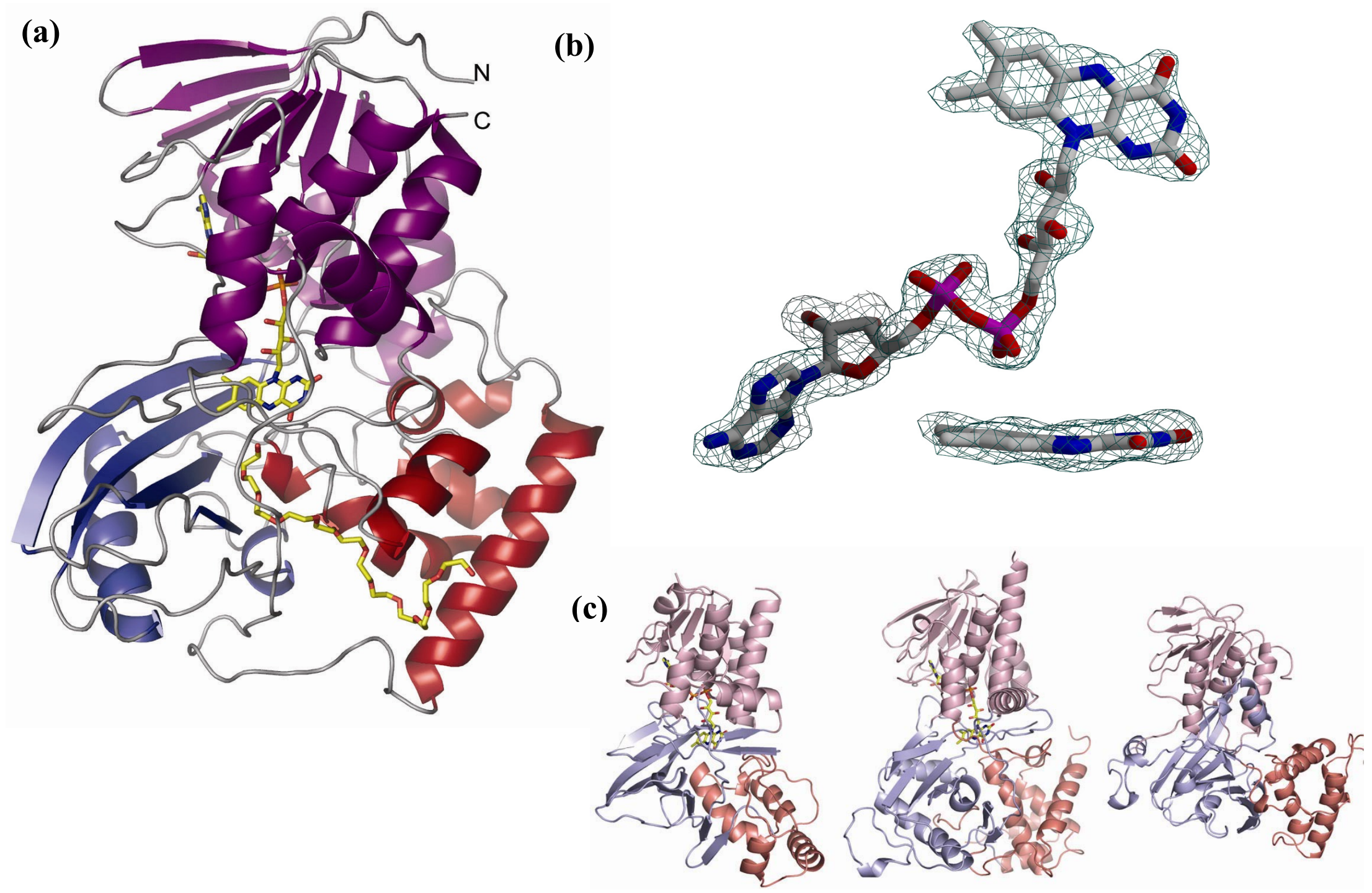
Fig. 16 PAI structure.

(a) Architecture of PAI with the FAD-binding domain colored in magenta, domain 2 in red, and domain 3 in blue. FAD and PEG400 are shown as stick models in the figure. (b) PAI structural analogs: polyaminooxidase (PDB ID code 1RSG) (Left), UDPgalactopyranose mutase (1I8T) (Center), and guanine nucleotide dissociation inhibitor (1GND) (Right). The domains are colored as for PAI. (c) A-weighted $\mathrm{mF}_{\mathrm{o}}-\mathrm{DF}_{\mathrm{c}}$ omit electron density maps contoured at $2 \sigma$ of FAD and a side-on view of the isoalloxazine ring (on the inset below)

FAD, whereas domains 2 and 3 are responsible for substrate binding and specificity, as well as formation of the active site (see below).

\subsubsection{FAD cofactor}

Electron density maps calculated with unbiased SIRAS phases unambiguously revealed the FAD cofactor (Fig. 16b) oriented towards the active site with its re-face. FAD adopts an extended conformation with the isoalloxazine part placed at the interface between domains 1, 2 and 3 and the ribose ring hydrogen-bonded to domain 1.

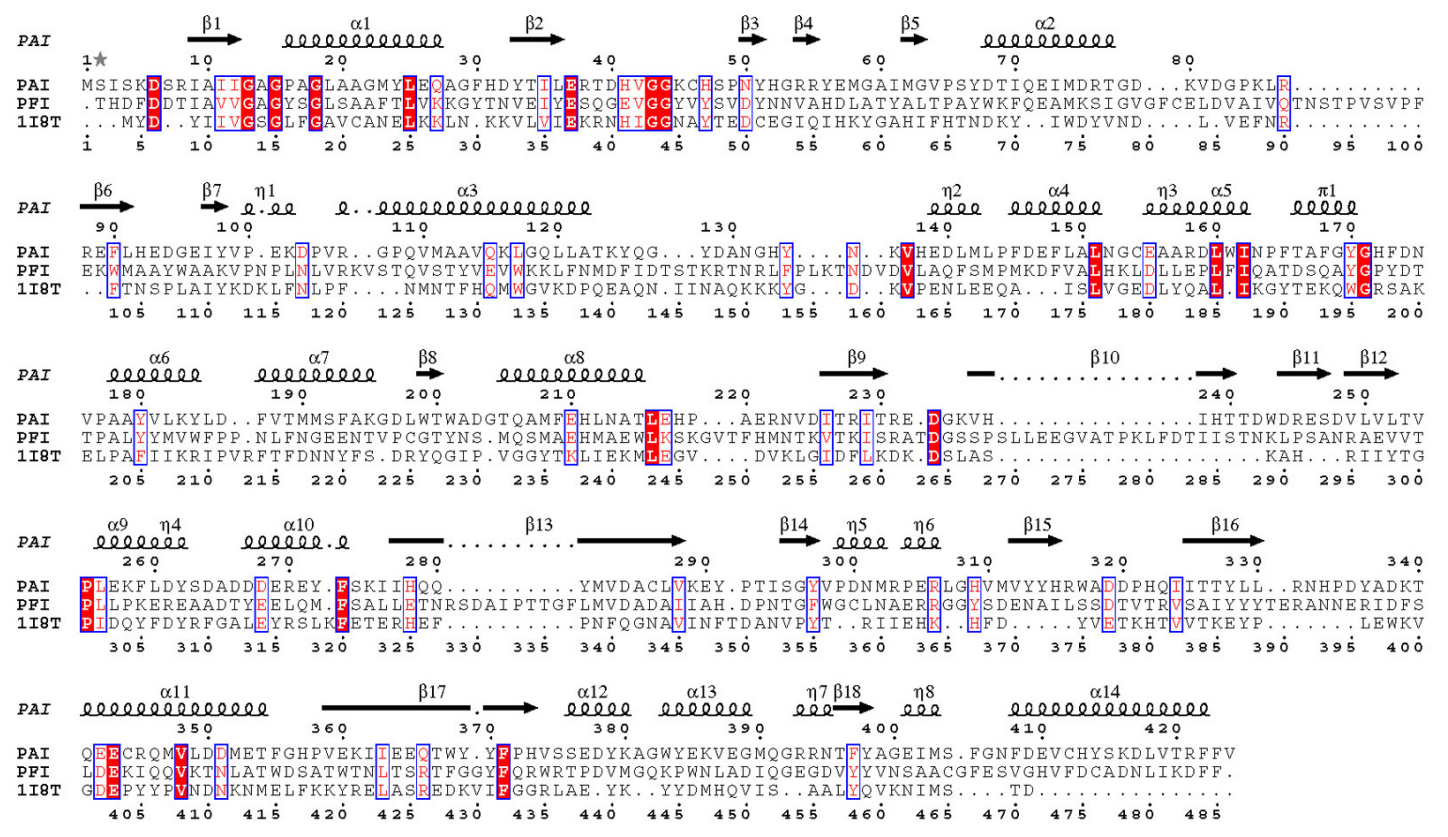

Fig. 17 Sequence conservation between PAI, PFI and polyamine oxidase.

Protein aminoacid sequence alignment of PAI, PFI and yeast PAO. The N-terminal FAD-binding motif is the most conserved in all three proteins. Secondary structure elements of PAI are shown in the top. Figure generated with ESPript program (Gouet 1999). 


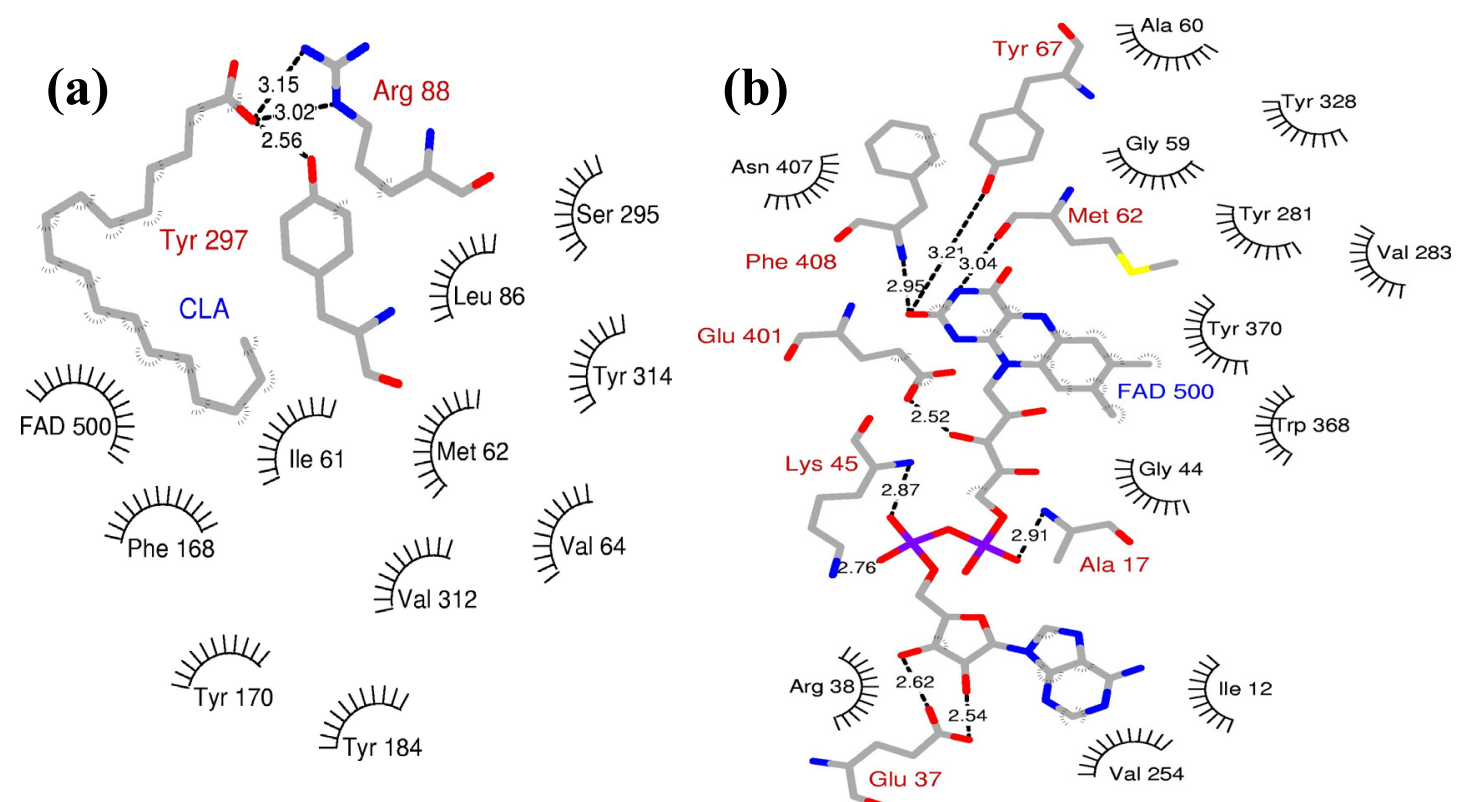

Fig. 18 Contacts of CLA (a) and FAD (b) in the active site.

As in other flavin-containing enzymes, the catalytically active isoalloxazine ring is buried in a hydrophobic pocket and inaccessible to bulk solvent. The isoalloxazine ring is bent in a so-called "butterfly" mode, such that the pyridoxine and xylene moieties adopt a $23^{\circ}$ angle (Fig. 16b). A similar deviation of an isoalloxazine ring from planarity was described for cholesterol oxidase (PDB ID 1MXT) and trimethylamine dehydrogenase (PDB ID 1DJN). While the flavin ring of FAD entertains only three direct hydrogen bonds with PAI, it is contacted by 9 hydrophobic residues with a total of 62 van der Waals contacts (Fig. 18b), illustrating the hydrophobic nature of the FAD binding pocket. In flavin-containing oxidases, the FAD N5 atom is usually hydrogen bonded to a protein main-chain or side-chain atom (Fraaije \& Mattevi 2000). This feature is absent in PAI, and the space in the vicinity of $\mathrm{N} 5$ is part of the substrate binding site from re-face of the isoalloxazine ring.

\subsubsection{Structure of PAI-CLA and PAI-CLnA complexes}

A putative substrate binding pocket was traced by a PEG400 molecule in the refined structure of free PAI (Fig. 16a). To reveal the exact binding mode of FAs, PAI was cocrystallized with LA (2BAB, Table 1) and LnA (2BAC). The identity of the bound FA (the substrate LA versus the product CLA) cannot be judged from the electron density as 
both LA and CLA can adopt a planar conformation of C9 to C13 carbons (Fig. 19a). In addition, the resolution of $2.2 \AA$ is not enough to distinguish $\mathrm{C}=\mathrm{C}$ bond length from the ordinary $\mathrm{C}-\mathrm{C}$ bond, otherwise direct identification of substrate/product from diffraction data would be possible. Thus, GC-MS analysis was used to confirm the nature of FA in the crystals (Fig. 20). In case of co-crystallization with LA, the crystals contained exclusively the product $(10 E, 12 Z)$-CLA (Fig. 20a), due to complete turnover of the substrate prior to crystallization. Assuming that orientation of LA in the active site is essentially the same as observed for the CLA product, the structure of PAI-product complex can be used to deduce the catalytic cycle and also illuminates the principles of substrate binding. CLA adopts a strongly bent U-shape when bound to PAI such that C1 and C18 are only $9.4 \AA$ apart (Fig. 19b). This bent conformation is imposed in part by conjugated double bonds. Protein also contributes to FA bending by positioning in the centre of substrate binding pocket Met62, which functions as a rigid pole around which the FA hydrocarbon chain wraps. The aliphatic tail of FA is contacted exclusively by hydrophobic amino acid side chains as well as FAD isoalloxazine ring (Fig. 18a). Only small conformational changes of PAI side-chains and FAD accompany FA binding, however they highlight important groups involved in direct hydrophobic contacts with the aliphatic chain. The isoalloxazine ring of FAD is displaced by up to $0.7 \AA$ relative to its position in the free PAI structure. Likewise, Phe168 rotates around $\mathrm{C}_{\gamma}$ by ca. $25^{\circ}$ and moves away from FA by up to $1.5 \AA$ compared to the free PAI structure. As a result, the pentadienyl moiety of FA is "sandwiched" between the aromatic rings of FAD and Phe168 in coplanar orientation (Fig. 19c). In this way, the site of hydrogen abstraction, C11 (see discussion for details), is only $3.2 \AA$ apart from electrophilic FAD atom N5. The carboxylate of CLA is hydrogen bonded to Arg88 and Tyr297, which shifts closer towards the FA by $0.4 \AA$ in the complex structure. At the entrance of the substrate binding channel Arg88 and Phe193 are $\pi$-stacked in parallel manner, excluding water from the active site (Fig. 19c, d, see below). In total, FA carboxyl entertains four hydrogen bonds: to water 128, Tyr297 and Arg88.

The structure of PAI-CLnA complex comprises all salient features of PAI-CLA complex with the exception of the CLnA methyl end, which apparently is mobile as judged from increased B-values in this region (Fig. 19d). Structural plasticity is also 


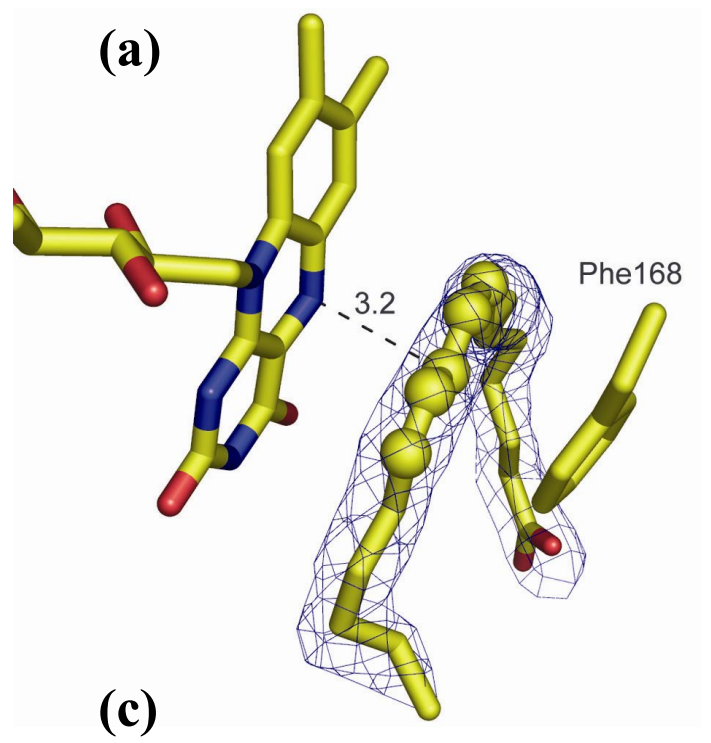

(b)

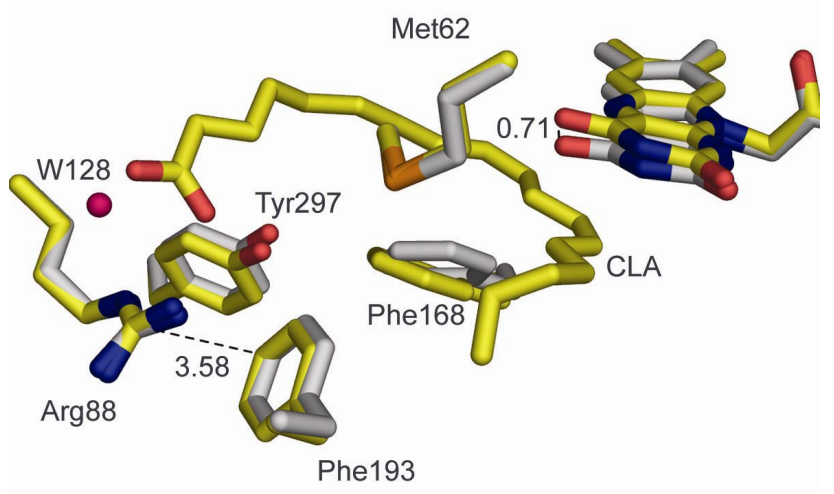

(d)
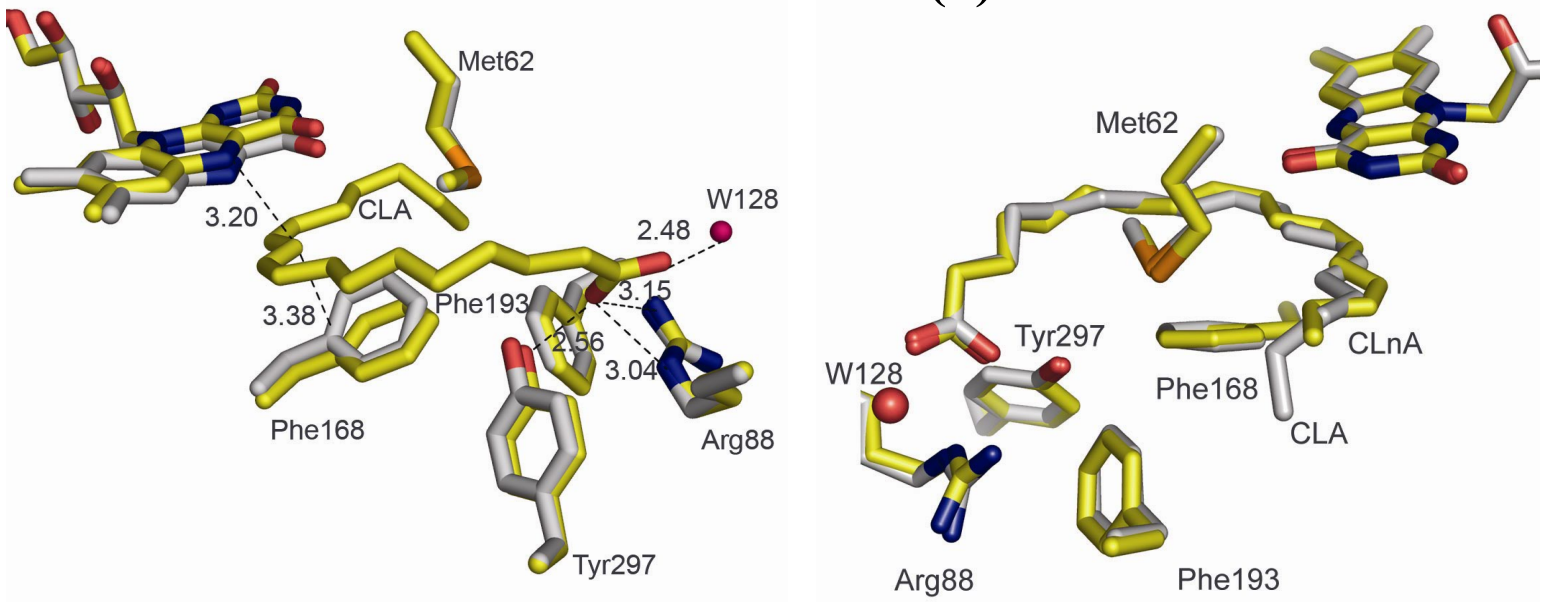

Fig. 19 Substrate binding to PAI.

(a) The $\sigma_{\mathrm{A}}$-weighted $\mathrm{mFo}-\mathrm{DFc}$ omit electron density map around the $(10 E, 12 Z)$-CLA is contoured at 2б. CLA adopts U-shaped form and atoms C9 to C13 are shown as spheres to visualize the planarity of conjugated double bonds system. (b) The PAI-CLA complex. Superposition of the active sites of apoPAI (grey) and bound to (10E,12Z)-CLA (yellow). Phe168, FAD and Tyr270 undergo small conformational change upon substrate binding; side chain of Phe168 is rotated and becomes coplanar with conjugated double bonds of CLA. FAD aromatic ring is shifted backwards to accommodate fatty acid and OH-group of Tyr270 moves towards carboxylate of CLA. (c) The view of active site after $180^{\circ}$ rotation in CLA plane. (d) Structure of PAI in complex with $(11 E, 13 E, 15 Z)$-LnA (CLnA, yellow). The structure of PAI-CLA complex is shown in gray for reference. The conjugated triene bond system is planar (atoms $\mathrm{C} 10$ to $\mathrm{C} 16$ ) and fatty acid molecule is more strongly bent compared to CLA. 
apparent for the conjugated triene moiety of CLnA, which is displaced by $0.6 \AA$ relative to CLA. Such FA shifts may be required for the efficient de- and re-protonation during catalysis. Importantly, a $418 \AA^{3}$ cavity is located next to the methyl end of FA, which could accommodate two more carbon atoms, possibly extending the substrate palette for PAI with FA containing twenty carbons (Hornung et al. 2005).

\subsubsection{Gating mechanism for substrate entry}

In all FAD-containing proteins the cofactor is buried inside the protein core, which requires the substrate to enter through a channel that can be as long as $30 \AA$. Three structures of PAI (2B9W and 2B9X, and 2BA9) were determined from crystals of space groups $\mathrm{I}_{1} 3$ and C2 (Table 1), and in all of them a PEG400 molecule was present. PEG400 is located inside the tunnel between domains 2 and 3 that stretches from the surface of PAI towards the FAD cofactor (Fig. 21a). Mostly hydrophobic residues form the tunnel surface, congruent with the hydrophobic nature of PAI substrates. Since PEG400 does not make hydrophilic contacts and there are no other significant cavities connected to substrate binding pocket, PEG400 mimics a FA hydrocarbon chain and, therefore, precisely delineates the path to the substrate binding site. However, PEG400 is neither a substrate nor a product analogue, unlike CLA or LnA, since it has no polar head group and cannot completely enter the active site (see above). In the "apo-structure" (meaning in this context that no large molecules are found in the substrate binding tunnel) two molecules of 1,4-butanediol and several waters roughly trace the PEG400 path (2B9Y, Table 1).

Flavoproteins can exclude water from the active site by various gating mechanisms that include domain movements, mobile loops, and also side-chain switches (Fraaije \& Mattevi 2000). Comparison of the PEG400-bound structures with the apo-structure and the structure of PAI-CLA complex illustrates that the latter option is realized in PAI. Superposition of these structures showed that, near PAI surface, Arg88 and Phe193 change their side-chain conformation when PEG400 enters the tunnel and, thus, function as gating residues (Fig. 21c). Up to four water molecules enter the active site in the vicinity of the FAD isoalloxazine moiety upon PEG400 binding, presumably due to the "gate" opening. Upon substrate binding and also when PEG400 is replaced by the smaller 1,4-butanediol molecule, these water molecules are absent from the active site. Exclusion 
(a)

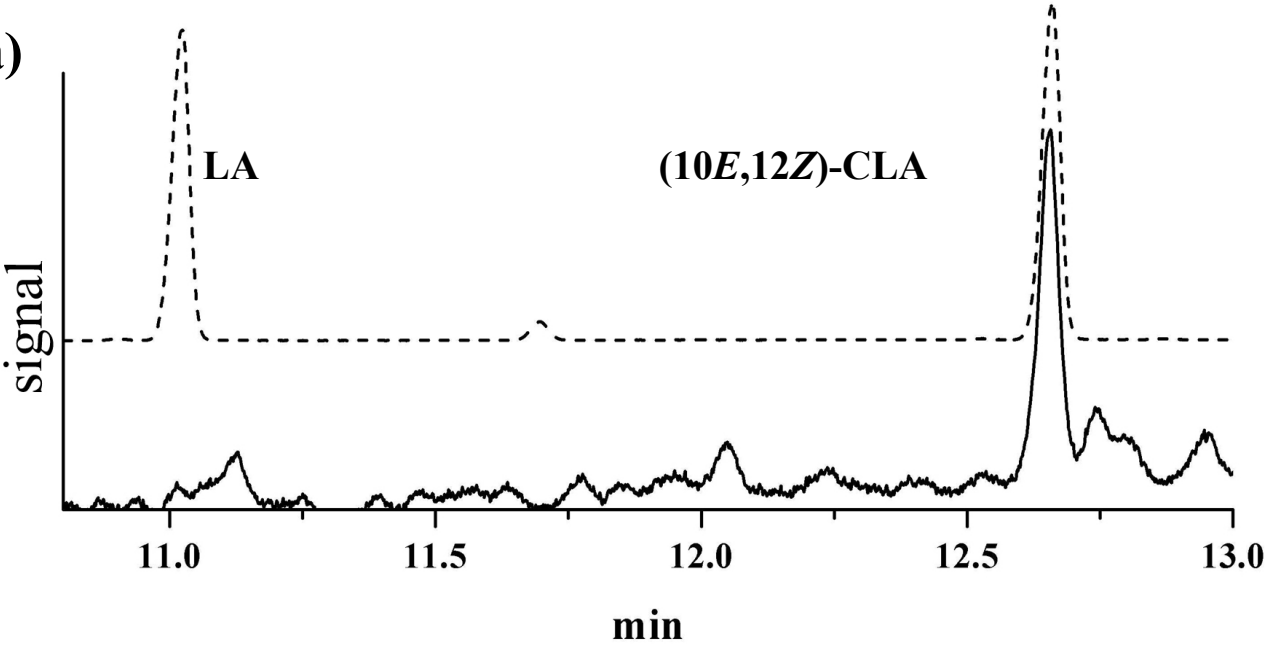

(b)

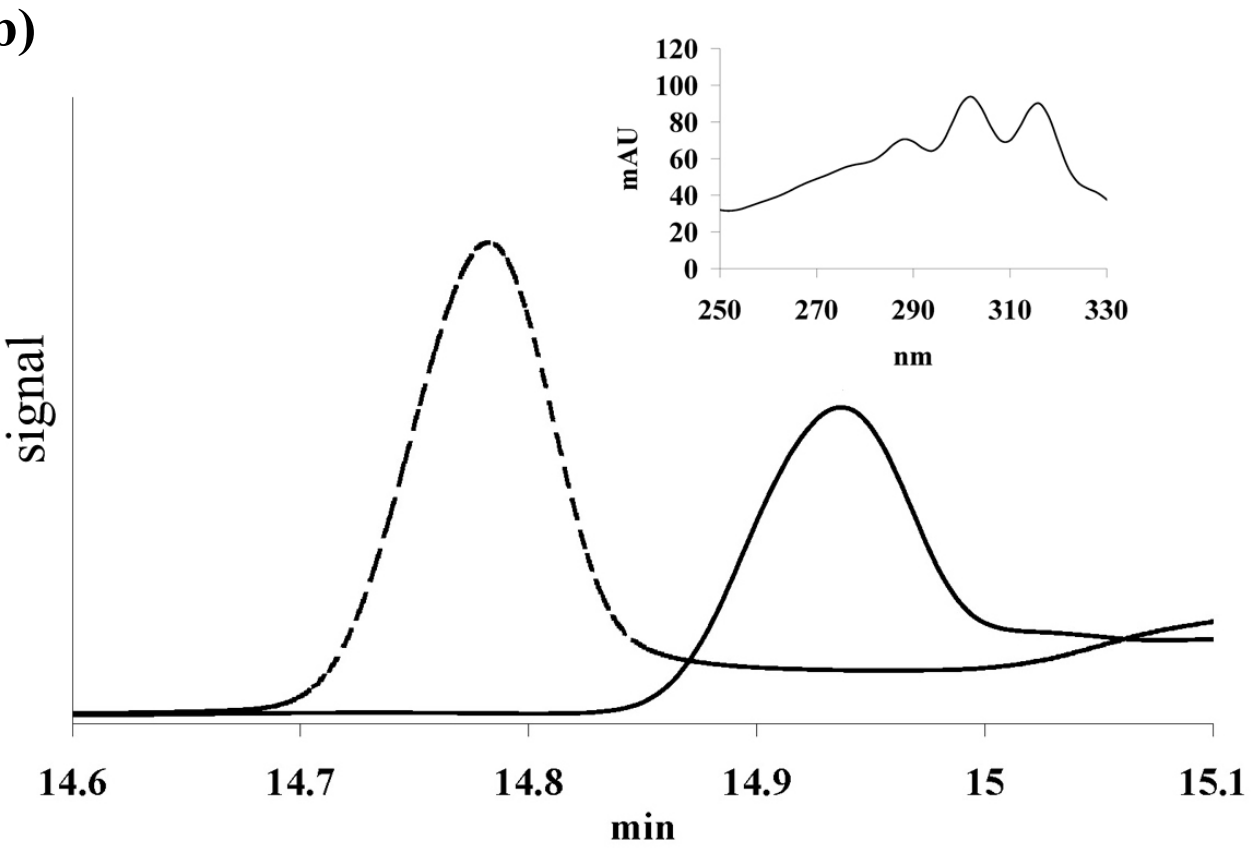

Fig. 20 Analysis of the fatty acid content in PAI crystals obtained after co-crystallization of PAI with LA and CLnA.

(a) The standard chromatogram (dashed line) shows LA and (10E,12Z)-CLA at retention times of 11.1 and 12.7 minutes, respectively. Only $(10 E, 12 Z)$-CLA is present in the complex crystals (bold line), suggesting that LA is quantitatively converted to the product $(10 E, 12 Z)$-CLA during co-crystallization. The amount of crystal-derived $(10 E, 12 Z)$-CLA sample was very small, therefore the scale is about 30 times less than for standard (y-axes are omitted for clarity) (b) Silver-ion HPLC chromatogram of LnA (dashed line) and CLnA extracted from crystals (bold line). On the inset: the identity of CLnA as conjugated triene is proved by UV-spectrum showing characteristic triplet of absorption maxima. 
of water by substrate molecule is essential to prevent side-reactions with electrophilic intermediates. A similar water exclusion mechanism by substrate binding is often exploited by enzymes, e.g. it was described in acyl-CoA dehydrogenases (Kim et al. 1993). Furthermore, electrostatic potential map of PAI surface reveals a positively charged area (residues Lys85, Arg87, Lys102, and Arg195) near the tunnel entrance (Fig. $21 \mathrm{~b}$ ). This positive patch may serve as an initial recognition site for carboxylic group of FA.

In the presence of PEG400 the side-chains of Arg88 and Phe193 are oriented away from the channel in the "open" position. However, in the apo-form or after FA binding, these residues swing back into the "closed" position and block the entry channel to the active site (Fig. 21c). The two positions are characterized by a rotation of the Phe193 side-chain by $78^{\circ}$ around $\mathrm{C}_{\gamma}$ and a reorientation of the $\operatorname{Arg} 88$ side-chain, which leads to distances of $11.1 \AA$ and $3.3 \AA$, respectively, between the tips of each side-chain in the open and closed states.

Arg88 can hydrogen bond to the carboxylate group of the substrate and Phe193 switches back to the 'closed' state and stacks onto the guanidinium group of Arg88 in a $\pi$-cation interaction. In addition, the hydrocarbon chain of the bound substrate displaces Phe193 from the 'open' position. Thus, the gate closure can be driven by FA binding.

\subsection{PAI reaction mechanism}

\subsubsection{Structure-based mechanism of FA isomerization}

Isomerization of double bond in LA by PAI requires high activation energy, taking into account $\mathrm{C}-\mathrm{H}$ and $\mathrm{C}=\mathrm{C}$ bond energies in aliphatic hydrocarbons (413 and $614 \mathrm{~kJ} / \mathrm{mol}$ respectively) and the absence of any activating groups near the double bonds. The reaction must proceed via some activated intermediate, e.g. a pentadienyl radical or carbcation resulting from FA after hydrogen removal from $\mathrm{C} 11$. The former activation mechanism is well known for LOX enzymes, however the reaction involves oxygen and yields oxidized FA or so-called oxylipins (Feussner \& Wasternack 2002, Gaffney et al. 1995). As mentioned in the introduction, both BFI and PFI remove a hydrogen atom at the $\mathrm{C} 11$ position when incubated with isotopically labeled substrates. The structure of PAI-CLA complex, where $\mathrm{C} 11$ of FA is in front of $\mathrm{N} 5$ atom of FAD, immediately leads to the working hypothesis that the latter acts as a redox catalyst. Given the space 
(a)

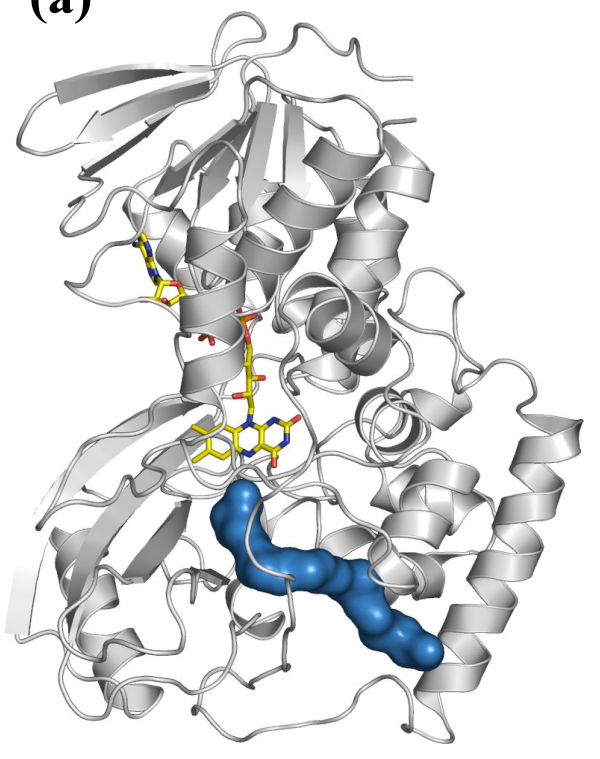

(b)

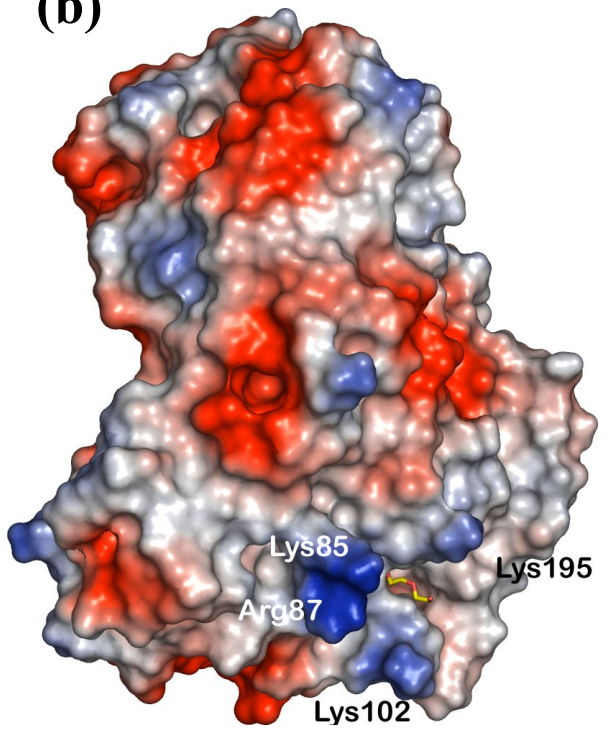

(c)

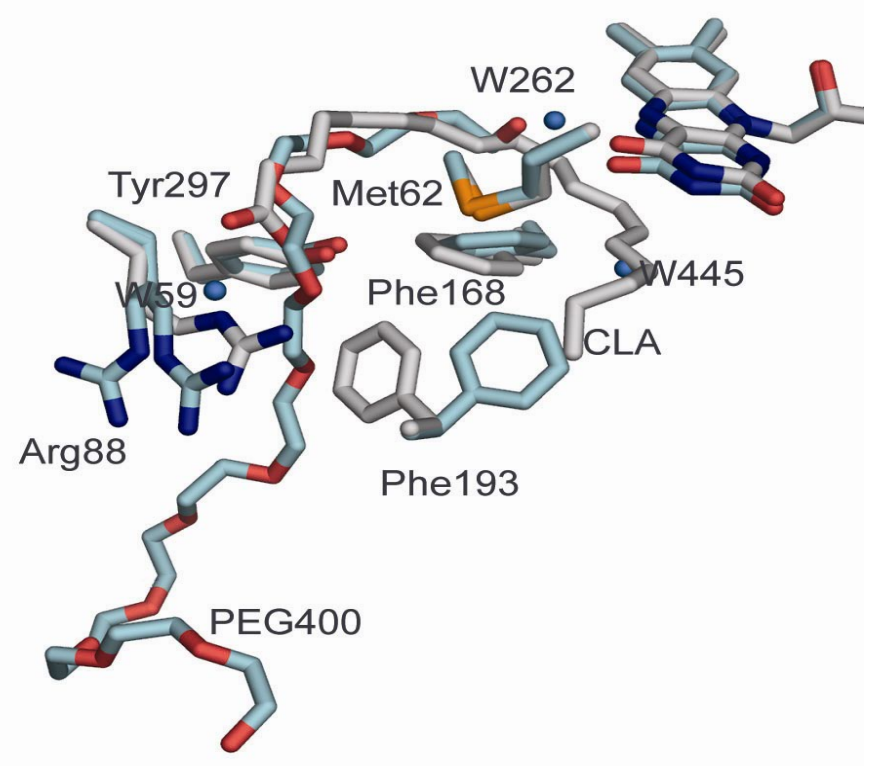

Fig. 21 Substrate entry channel and gating mechanism in PAI.

(a) The surface representation of part of the PEG400 molecule bound to PAI in the absence of substrate/product shows the $30 \AA$ path from the surface to the active site FAD (drawn as sticks). (b) The surface potential of PAI shows an electropositive area localized at the entrance of the tunnel that is created by Lys85, Arg87, Lys102, and Lys195. The PEG400 molecule marks the entry of the tunnel.

(c) Conformational changes in active site associated with PEG400 binding reveal the gating mechanism. PEG400 Phe193 and Arg88 are in the "open" conformation when PEG400 is bound (blue) compared to the apo enzyme (grey) 


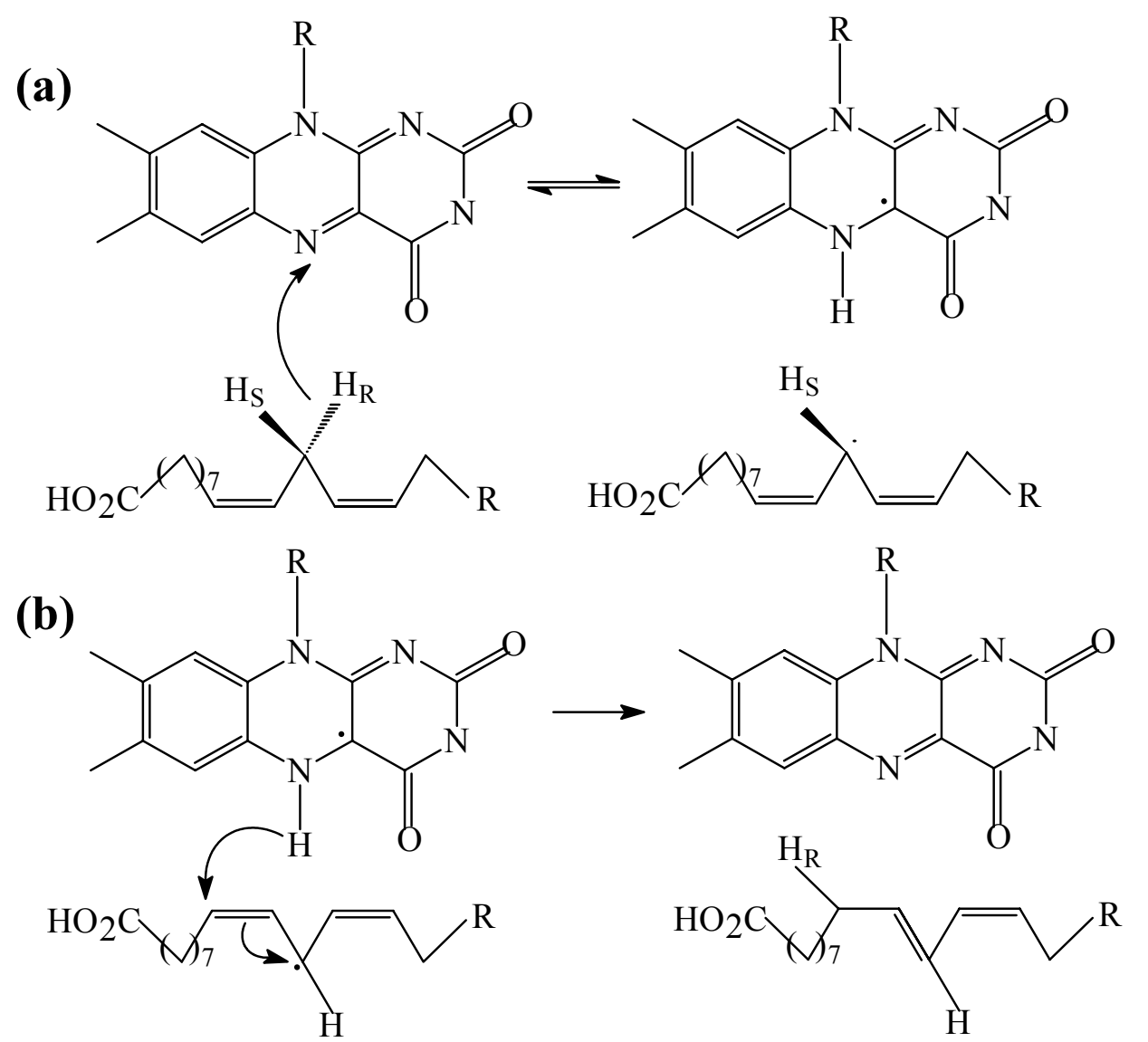

Fig. 22 Structure-based isomerization mechanism of LA to $(10 E, 12 Z)$-CLA.

(a) Pro- $R$ Hydride abstraction by FAD atom N5 yields an intermediate LA carbocation, which is stabilized by $\pi$-conjugation. (b) Hydride transfer from FAD atom N5 to the Re-side of LA atom C9 yields CLA and regenerates the oxidized form of FAD.

restrictions in the binding site of PAI (Fig. 19d), any movements of the substrate and the products should be on a small scale of ca. $2 \AA$. Therefore, C11 pro- $R$ hydrogen of LA would point directly towards N5 of FAD in the initial PAI-substrate complex, ready for abstraction at the first step of isomerization reaction (Fig. 22a).

The main issue regarding isomerization of PUFAs by PAI is whether the catalytic mechanism is radical or ionic. Theoretically, both mechanisms are equally plausible and to date several flavoenzymes catalyzing reactions with no redox change via one- or twoelectron transfer are known (Bornemann 2002). After either hydrogen atom or hydride abstraction by FAD, the radical or cationic FA intermediate would be stabilized by $\pi$ conjugation. Additional stabilization of a cationic intermediate would be provided by the 
interaction with aromatic systems of FAD and Phe168. Likewise, the negative charge on FAD can be efficiently stabilized by PAI. In many redox active flavoenzymes the N1$\mathrm{C} 2=\mathrm{O} 2$ locus of isoalloxazine ring is in ca. $3.5 \AA$ distance to a positively charged residue or the N-terminus of an $\alpha$-helix dipole, which would stabilize a negative charge on FAD during catalysis (Fraaije \& Mattevi 2000, Ghisla \& Massey 1989). In PAI, such a charge would be stabilized by the dipole of the C-terminal $\alpha$-helix (residues F408-F422), which has counterparts in UDP-mutase and PAO (Fig. 16b).

Whether carbcation or radical, the activated intermediate should spontaneously isomerize to energetically favorable conjugated diene. In the absence of spatial restrictions this process must produce equimolar amounts of $(10 E, 12 Z)$ - and $(9 Z, 11 E)$ CLA. However, in PAI Phe168 is shifted towards C9 rather than C13 of CLA (distance to $\mathrm{C}_{\gamma}$ of Phe168 is 3.9 and $4.6 \AA$ respectively). Thus, the enzyme may determine the isomerization route by preferential stabilization of partial positive charge on C9. Readdition of hydrogen from N5 of FAD to C9 completes the reaction cycle. For this hydrogen addition, either the substrate or FAD must shift by $2.6 \AA$ relative to the $\mathrm{C} 11$, bringing $\mathrm{C} 9$ in front of N5 of FAD. As FAD is tightly bound to PAI by numerous interactions (Fig. 18b) the intermediate is likely to move after hydrogen abstraction. In line with this hypothesis the structural plasticity of the bound FA is seen from comparison of the two product complexes (Fig. 19).

Interestingly, PAI was shown to catalyze conjugated triene formation from LnA but not from $\gamma$-LnA $\left(18: 3^{\Delta 6 Z, \Delta 9 Z, \Delta 12 Z}\right.$ ) (Hornung et al. 2005), the double bonds in $\gamma$-LnA are shifted by 3 carbon atoms towards the carboxy-terminus compared to LnA. Two cycles of hydrogen abstraction-isomerization would be required for conjugated triene formation in either case. In case of $\gamma$-LnA, formation of $(10 E, 12 Z)$-diene would leave C6 double bond two carbons apart and no further allylic intermediates are possible. In contrast, such allylic intermediate still can be formed from $(10,12,15)$-triene system of partially isomerised LnA. Apparently, absolute product specificity of $(10 E, 12 Z)$ - over $(9 Z, 11 E)$ CLA displayed by PAI enables double bond shift only towards $\omega$-terminus of PUFAs.

\subsubsection{Hydrogen transfer during LA isomerization}

5,5-dimethyl-oxazoline (DMOX) derivatives of PUFAs display characteristic mass spectra indicating double bond position along the aliphatic chain (Christie 1998). 
Reaction products of PAI with LA isotopically labeled with deuterium at C11 were analyzed by GC-MS after conversion to DMOX derivatives. The pattern of DMOX(10E,12Z)-CLA fragmentation is shown in Fig. 23 (only the region of interest containing peaks of high $\mathrm{m} / \mathrm{z}$ is included). In non-labeled (10E,12Z)-CLA gaps of 12 a.m.u. between the fragments $\mathrm{C} 9-\mathrm{C} 10(\mathrm{~m} / \mathrm{z}=210$ and 222$)$ and $\mathrm{C} 12-\mathrm{C} 13(\mathrm{~m} / \mathrm{z}=236$ and 248$)$ enable to localize double bonds at positions 10 and $12 ; \mathrm{C} 11$ and $\mathrm{M}^{+}$have masses of $\mathrm{m} / \mathrm{z}=236$ and 333 respectively. When $(11 S)$-deuterio-LA was used as a substrate, deuterium remained at position $\mathrm{C} 11$, as indicated by +1 a.m.u increase in the $\mathrm{C} 11$ fragment (observed masses for C9-C12 fragments are 210, 222, 237, 249, and $\left.\mathrm{M}^{+}=334\right)$. However, in case of 11dideuterio-LA, the transfer of one deuterium from C11 to C9 is evident (observed masses for C9-C12 fragments are 211, 223, 238, 250, and $\mathrm{M}^{+}=335$ ), i.e. fragments $\mathrm{C} 9$ and $\mathrm{C} 10$ have +1 a.m.u. mass increase compared to non-labeled (10E,12Z)-CLA and all fragments higher than $\mathrm{C} 10$ have a +2 a.m.u increase. The isotopic ratio between hydrogen and deuterium was similar for the substrate and product, indicating no loss of deuterium during the isomerization reaction (the mass spectrum of 11-dideuterio-LA is not shown).

Reaction in ${ }^{2} \mathrm{H}_{2} \mathrm{O}$ did not lead to deuterium incorporation in the product (data not shown), ruling out both direct involvement of solvent in the reaction and hydrogen exchange between N5 of reduced FAD and water. In summary, these data confirm the structure-based hypothesis that the 11-pro- $R$-hydrogen is abstracted by FAD during LA isomerization and then transferred back to C9 forming (10E,12Z)-CLA.

Atomic motions during reaction can be indirectly accessed by measuring kinetic parameters with isotopically labeled substrates. Kinetic isotope effect (KIE) experiments with the substrates above are summarized in Table 2. The overall reaction rate judged by the $\mathrm{V}_{\max }$ value decreased from 43.67 to $8.27 \mathrm{nM} \mathrm{s}^{-1}$ for LA and 11-dideuterio-LA respectively, indicating 5.26 fold $\mathrm{KIE}$ due to deuteration at $\mathrm{C} 11 . \mathrm{V}_{\max }=35.09 \mathrm{nM} \mathrm{s}^{-1}$ for (11S)-deuterio-LA yielded secondary KIE value equal to $1.25 . \mathrm{K}_{\mathrm{m}}$ values for LA and (11S)-deuterio-LA agree within the experimental error, however, the $\mathrm{K}_{\mathrm{m}}$ value for 11dideuterio-LA is only $2.28 \mu \mathrm{M}$, which is approximately two times lower compared to LA.

The fact that even after prolonged incubation of PAI with (10E,12Z)-CLA no traces of LA were detected by GC (data not shown) confirms that isomerization of LA by PAI is irreversible process. Assuming that initial PAI-LA complex is irreversibly converted to 
(a)

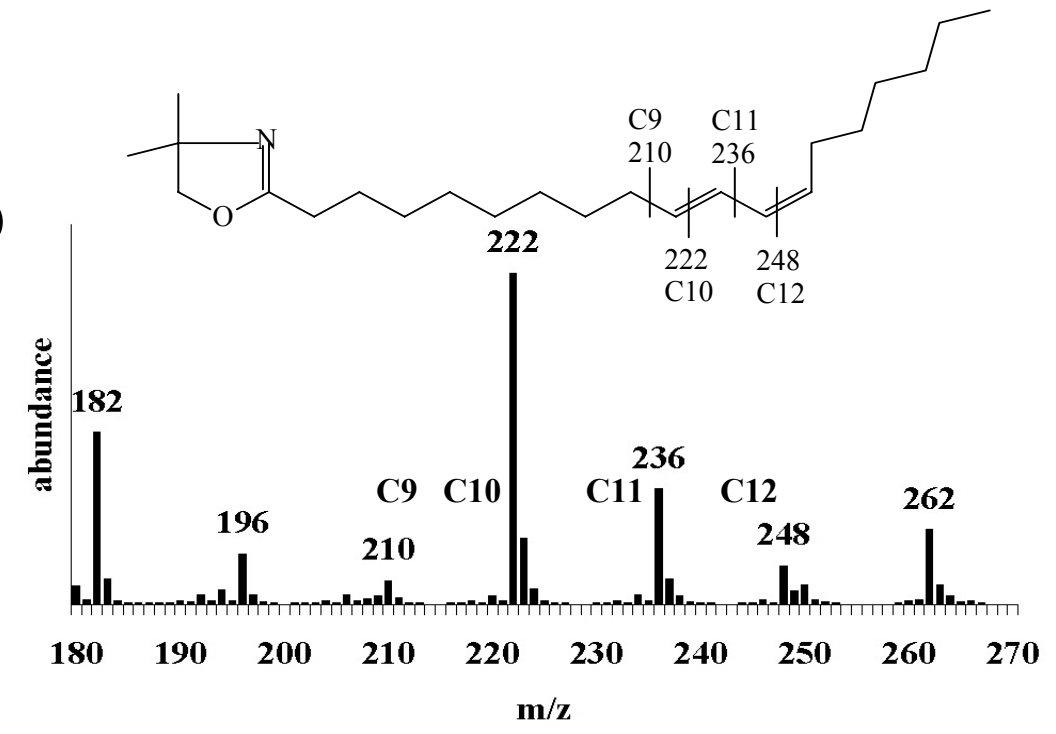

(b)

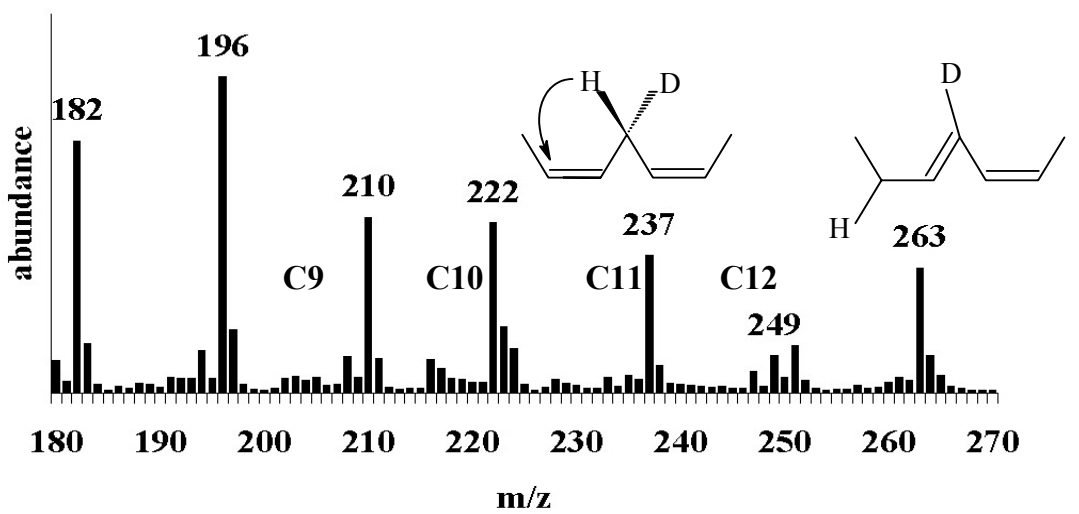

(c)

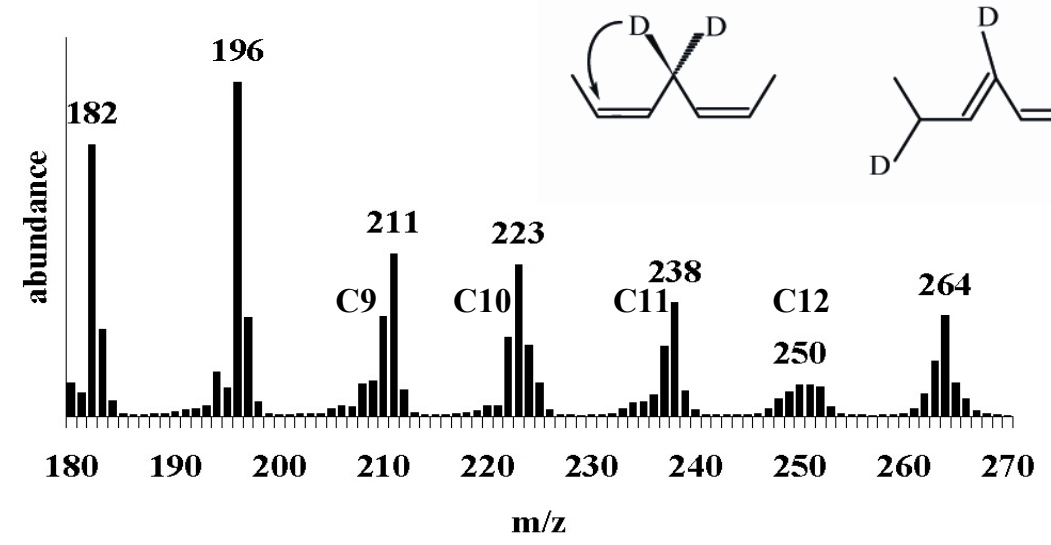

Fig. 23 Analysis of isotopic label migration in (10E,12Z)-CLA.

(a) Mass spectrum of $(10 E, 12 Z)$-CLA derived from non-labeled LA. Peaks corresponding to fragments C9-C12 are marked and the fragmentation pattern of DMOX-(10E,12Z)-CLA is shown on the insert.

(b) Mass spectrum of $(10 E, 12 Z)$-CLA derived from (11S)-deuterio-LA. On the inset - 11-proShydrogen is not abstracted by PAI. (c) Same as (b) but 11-dideuterio-LA was used as the substrate. On the inset - scheme of deuterium transfer by PAI. 
Scheme 1.

$$
\text { PAI }+\mathrm{LA} \underset{\mathrm{k}_{2}}{\stackrel{\mathrm{k}_{1}}{\rightleftharpoons}}[\mathrm{PAI}-\mathrm{LA}] \stackrel{\mathrm{k}_{3}}{\longrightarrow} \mathrm{PAI}+\mathrm{CLA}
$$

the PAI* $(10 E, 12 Z)-C L A$ complex at the rate determined by $\mathrm{k}_{3}$, the classical MichaelisMenten kinetic scheme consisting of three steps can be applied to PAI reaction (Scheme 1). Lower $K_{m}$ value for the deuterated substrate can be attributed to decreased $k_{3}$ and accordingly to the slower rate of step 2 (see also discussion).

Table 2. Kinetic parameters of PAI wt and mutant forms.

\begin{tabular}{|l|l|l|l|l|}
\hline Protein & Substrate & $\mathrm{Km}^{*}, \mu \mathrm{M}$ & $\mathrm{Vmax} * \mathrm{nM} / \mathrm{s}$ & $\mathrm{k}{ }^{*}, \mathrm{~s}^{-1}$ \\
\hline PAI wt & LA & $4.82 \pm 0.84$ & $43.67 \pm 3.40$ & $4.37 \pm 0.34$ \\
\hline PAI wt & $(11 S)$-deutero-LA & $4.08 \pm 0.52$ & $35.09 \pm 2.14$ & $3.51 \pm 0.21$ \\
\hline PAI wt & 11 -dideutero-LA & $2.28 \pm 0.55$ & $8.27 \pm 0.79$ & $0.83 \pm 0.08$ \\
\hline $\begin{array}{l}\text { PAI } \\
\text { R88S }\end{array}$ & LA & $65.70 \pm 8.04$ & $29.28 \pm 1.23$ & $2.93 \pm 0.12$ \\
\hline $\begin{array}{l}\text { PAI } \\
\text { F193A }\end{array}$ & LA & $37.94 \pm 7.88$ & $32.70 \pm 2.58$ & $0.33 \pm 0.26$ \\
\hline $\begin{array}{l}\text { PAI } \\
\text { R88SF193A }\end{array}$ & LA & $25.77 \pm 4.13$ & $15.52 \pm 0.60$ & $0.16 \pm 0.06$ \\
\hline
\end{tabular}

$* \mathrm{~K}_{\mathrm{m}}=\left(\mathrm{k}_{2}+\mathrm{k}_{3}\right) / \mathrm{k}_{1}$ (see Scheme 1$), \mathrm{k}_{3}=\mathrm{V}_{\max } /\left[\mathrm{E}_{\mathrm{t}}\right]$. $\mathrm{E}_{\mathrm{t}}$ is total enzyme concentration, which was $10 \mathrm{nM}$ for PAI wt, and other data were normalized to this value.

\subsubsection{Active site engineering by site-directed mutagenesis}

In order to investigate the role of two gating residues Arg88 and Phe193 on substrate recognition by PAI, these residues were mutated to small non-charged amino acids. The far reaching goal of these experiments was to change the strict substrate preference of PAI over free FA towards esterified FA and lipids. Therefore, the purified mutant enzymes (Fig. 24) were initially screened for their ability to isomerize the methyl ester of LA (measured by GC as ratio of product to substrate peak areas, Suppl. Table 4). Kinetic 
data for the most active mutant enzymes with LA as a substrate are summarized in Table 2. Mutation of Arg 88 to Ala inactivated PAI to the extent that activity could not be measured spectrophotometrically. Single exchanges of Arg88 to Ser and Phe193 to Ala, as well as the combination of both, caused pronounced $\mathrm{K}_{\mathrm{m}}$ increase ranging from 5-fold to 13 -fold, i.e. substrate binding is indeed strongly compromised in the absence of gating residues. $\mathrm{V}_{\max }$ is decreased 25 and $30 \%$ for Ser88 and Ala193 mutations, respectively, and it is $60 \%$ lower for the double mutant than for wt PAI. This pronounced effect of mutations on $\mathrm{V}_{\max }$ indicate that the rate of step 2 (Scheme 1) also depends on interaction of LA carboxyl with gating residues (see discussion for details).

The stabilizing role of Phe168 on the intermediate formation and its rearrangement was discussed in 3.2.1. Replacing Phe168 by a relatively bulky hydrophobic Ile rendered the enzyme inactive, underscoring the role of aromatic ring of Phe for the catalysis. Shifting Phe168 relative to FAD might alter the direction of intermediate isomerization resulting in $(9 Z, 11 E)$-CLA or mixture of both $(9 Z, 11 E)$-CLA and $(10 E, 12 Z)$-CLA. The mutant protein in which Phe168 and neighboring Gly169 were switched turned out to be inactive as well. These two experiments highlight the crucial role of Phe168 for PAI activity.

Since FAs are found in vivo mostly as esterified species, targeting of isomerase protein to cell membrane might result in an enhanced yield of CLA due to the direct contact between the protein and membrane lipids. The expression of PAI in eukaryotic hosts resulted in low levels of $(10 E, 12 Z)$-CLA accumulation in the free FA pool but not in esterified FAs (Hornung et al. 2005). The N-terminal C2 domain found in many LOX enzymes (Oldham et al. 2005, Skrzypczak-Jankun et al. 2001, Minor et al. 1996, Gillmor et al. 1997) is responsible for the anchoring of catalytic domain to the lipid bilayers (Oldham et al. 2005, May et al. 2000). Therefore the C2 domains either from the cucumber 13-LOX (May et al. 2000) or the rabbit 15-LOX (Gillmor et al. 1997) were fused to the N-terminus of PAI and the resulting proteins overproduced in E. coli and tested for activity (Fig. 24). Both chimeric enzymes produce (10E,12Z)-CLA from LA (Fig. 24b), although to a lesser extent than wt PAI. No activity was found when $20 \mu \mathrm{M}$ TLA or di-linoleyl-PC were used as substrates (data not shown). 


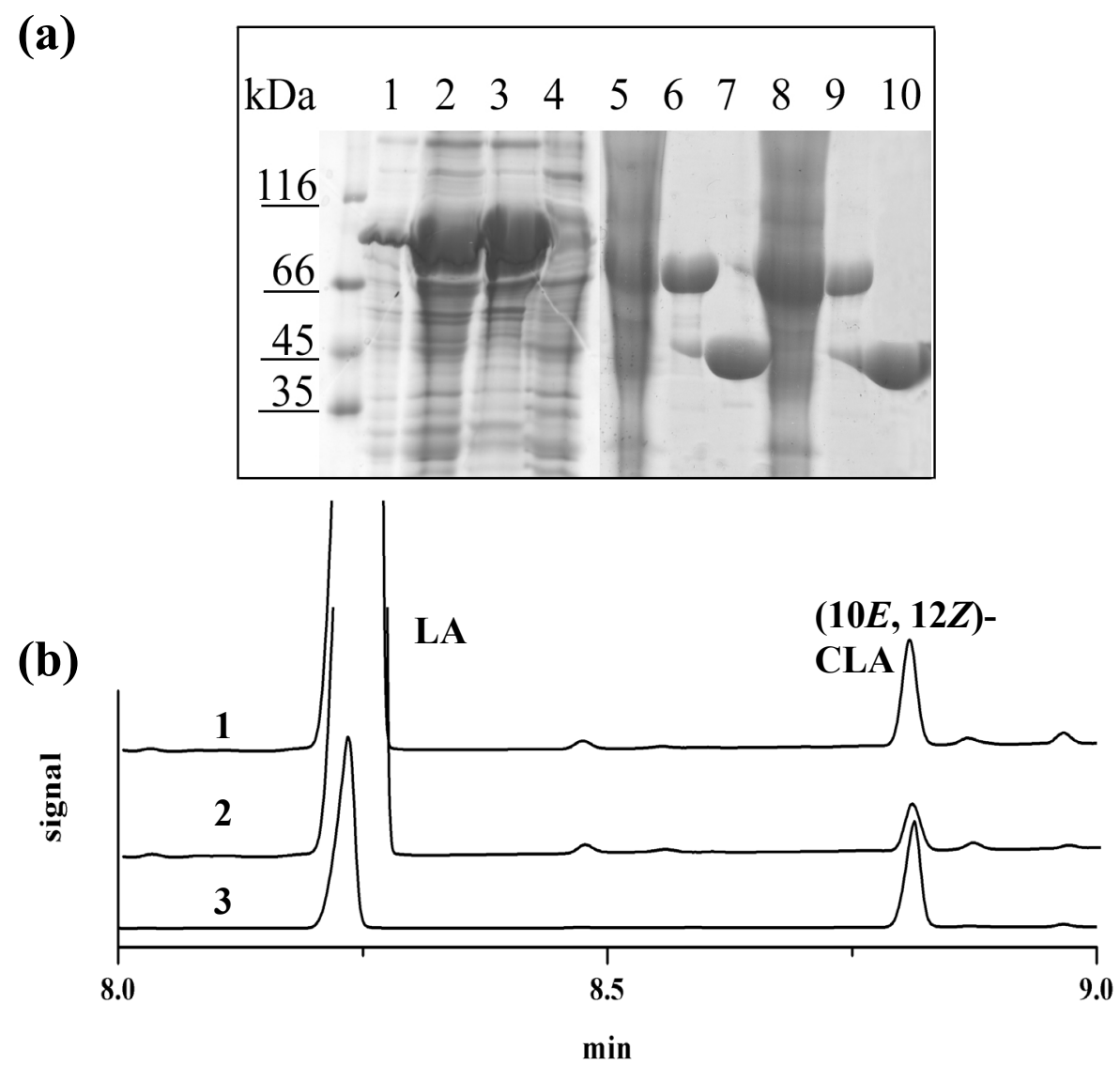

Fig. 24 Overexpression of LOX-PAI fusions and PAI point mutants.

(a) 1 - E. coli cells transformed with GST-PAI fusion, 2 - N-terminal lipid binding domain of CsLOXPAI fusion, 3 - N-terminal lipid binding domain of OcLOX-PAI fusion, 4 - pET28 empty vector control; 5 - GST-PAI R88S cell lysate, 6 - GST-PAI R88S eluted from GSH-Sepharose, 7 - PAI R88S after ion exchange column; 8-10 are the same purification steps for PAI R88S+F193A mutant protein. (b) GC analysis of reaction products of CsLOX-PAI and OcLOX-PAI with LA. 1 - CsLOX-PAI cell lysate + LA, 2 - OcLOX-PAI cell lysate + LA, 3 - GST-PAI cell lysate + LA. Peaks of LA and $(10 E, 12 Z)$-CLA are labelled, the graphs 1 and 2 are scaled up to show the product peak.

\subsubsection{Enzyme-substrate complex formation, anaerobic reduction and cofactor exchange}

To assess the flavin state during the reaction course, the effects of substrate binding and anaerobic reduction on PAI absorption spectrum were assayed. Addition of LA (both aerobic and anaerobic) turns the color of PAI solution from yellow to orange-red, and an additional peak appears in the spectrum around $545 \mathrm{~nm}$ (Fig. 25a). The color returns to yellow by repeated cycles of dilution-ultrafiltration. Thus, most likely these spectral 
(a)

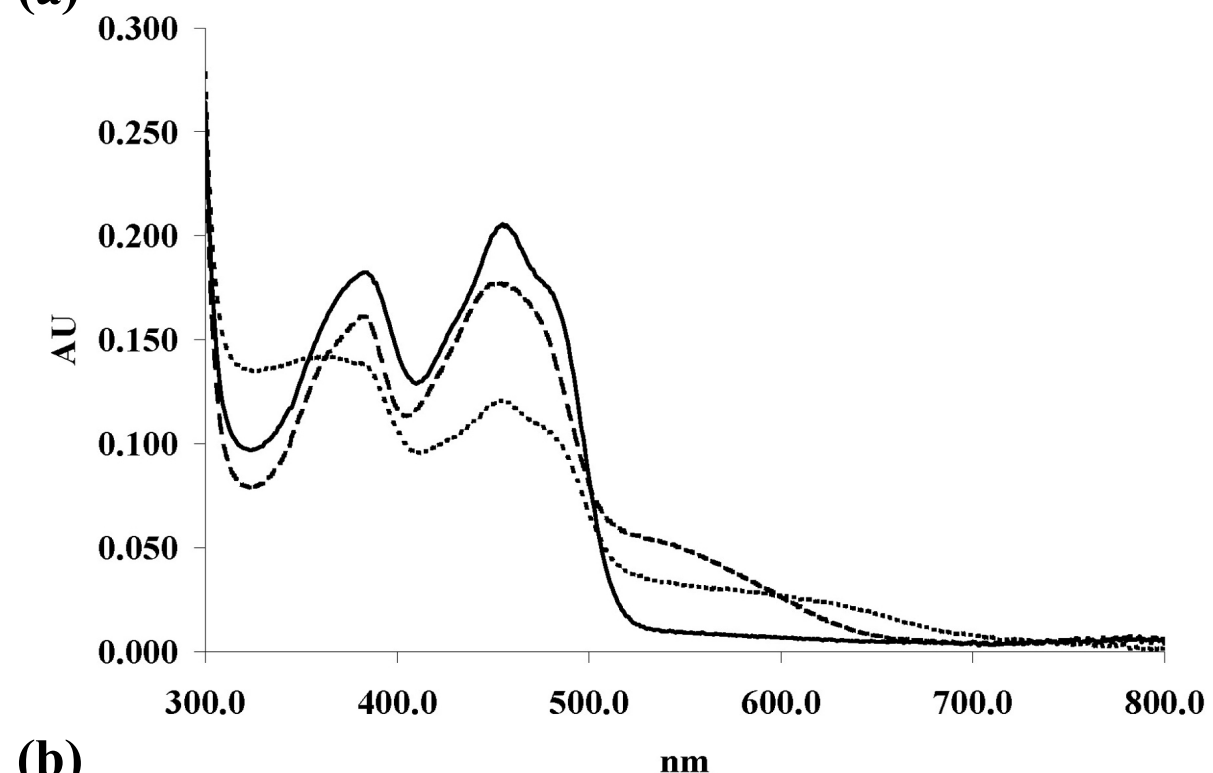

(b)

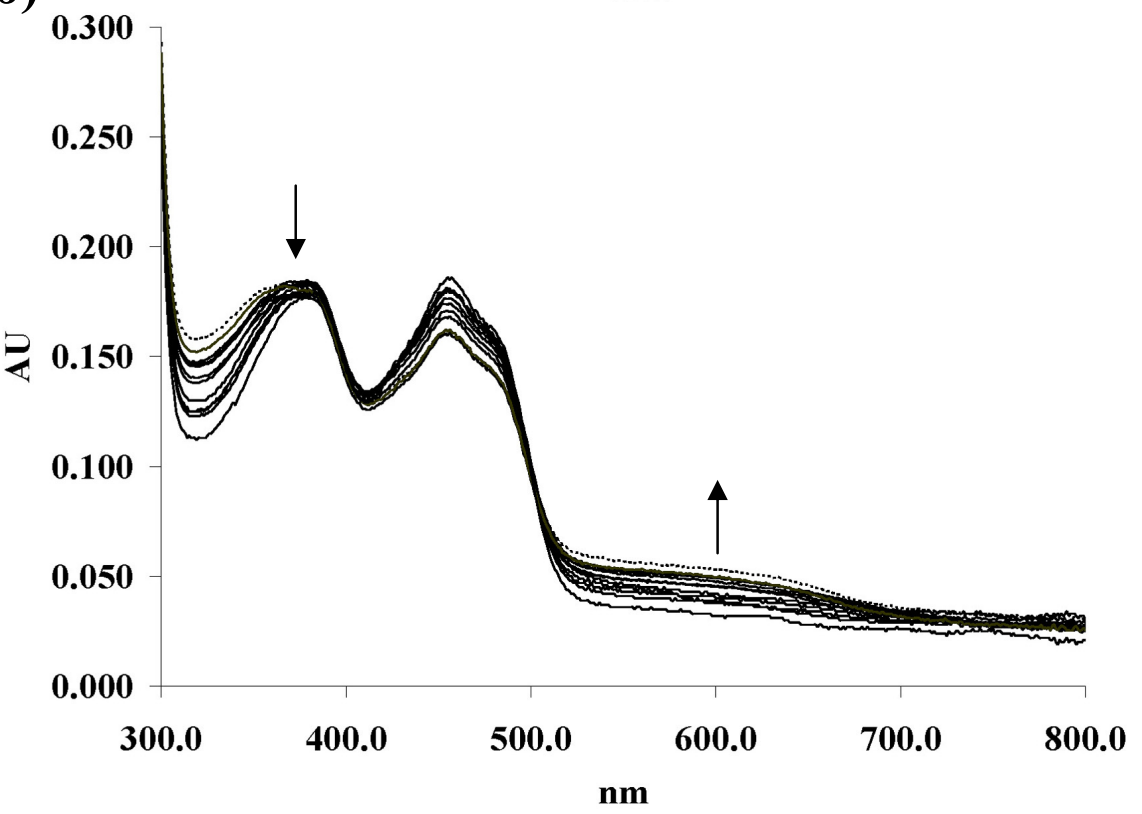

Fig. 25 Spectral changes upon PAI-CLA complex formation and PAI reduction.

(a) The spectra of oxidized PAI in free form (solid line) and upon addition of 10x molar excess of LA (dashed line), note the additional peak around $550 \mathrm{~nm}$ in this case. The spectrum of reduced PAI (dotted line) is given for reference. (b) Anaerobic photoreduction of PAI. The spectra were recorded after each pulse of irradiation. Decrease of absorbance at 454 as well as increase at $600 \mathrm{~nm}$ is indicated by arrows, dotted line - the spectrum after 60 min of irradiation. Even after extensive irradiation PAI is not fully reduced. 
changes are due to the charge-transfer interaction between FAs and oxidized FAD. The involvement of reduced FAD is unlikely, because this species is unstable on air. Real charge-transfer complexes involve electron-rich and electron deficient partners, since both LA and (10E,12Z)-CLA are neutral molecules, the absorption probably reflects the effect of $\pi$-stacking interaction between Phe168, double bonds of FAs and FAD isoalloxazine ring.

Anaerobic photoreduction of PAI by EDTA, sodium oxalate or Tris in the presence of 5-deaza-5-carba-FAD likely produces a neutral FAD radical as indicated by an absorption increase at $600 \mathrm{~nm}$ (Fig. 25b). The neutral protonated flavin semiquinone absorbs around 580-600 $\mathrm{nm}$ with extinction coefficient about $4000 \mathrm{M}^{-1} \mathrm{~cm}^{-1}$ (Chapman 1999). Even after extensive irradiation this long-wavelength absorbance is not decreased, which can be attributed either to an accumulation of a semiquinone radical or to the absorbance of reduced FAD. Direct monitoring of radical formation by EPR would be helpful to discriminate against these possibilities.

To test whether the radical mechanism is indeed employed by the enzyme, the activity of PAI reconstituted with 5-deaza-5-carba-FAD was probed. It should be noted that convenient methods of FAD removal like ammonium sulphate precipitation under acidic $\mathrm{pH}$ and dialysis against 1-2 $\mathrm{M} \mathrm{KBr}$ did not release FAD from the holoprotein, which is not surprising taking into account that isoalloxazine moiety of FAD is deeply buried in the hydrophobic active site (Fig. 18b). Only refolding of protein from $6 \mathrm{M}$ guanidium chloride by gel-filtration method resulted in apo-PAI with the yield about $30 \%$. After reaction of apo-PAI with LA in the presence of 5 fold molar excess of either FAD or 5-deaza-5-carba-FAD products were analyzed by GC (Fig. 26a). The amount of the sample injected was intentionally set very high, so that trace amounts of CLA could be detected with apo-PAI and 5-deaza-5-carba-FAD-PAI. However, only PAI-FAD complex gives the detectable amounts of the product (Fig. 26a, trace 3). The incorporation of 5-deaza-5-carba-FAD in apo-PAI was confirmed by UV-Vis spectrum of the reconstituted enzyme (Fig. 26b). Since 5-deaza-5-carba-FAD acts only as twoelectron redox catalyst it is generally assumed that enzymes operating via radical mechanism are inactivated by such replacement. The redox potential of 5-deaza-5-carbaFAD for two-electron transfer is $100 \mathrm{mV}$ lower compared to FAD (-310 and $-208 \mathrm{mV}$ 
(a)

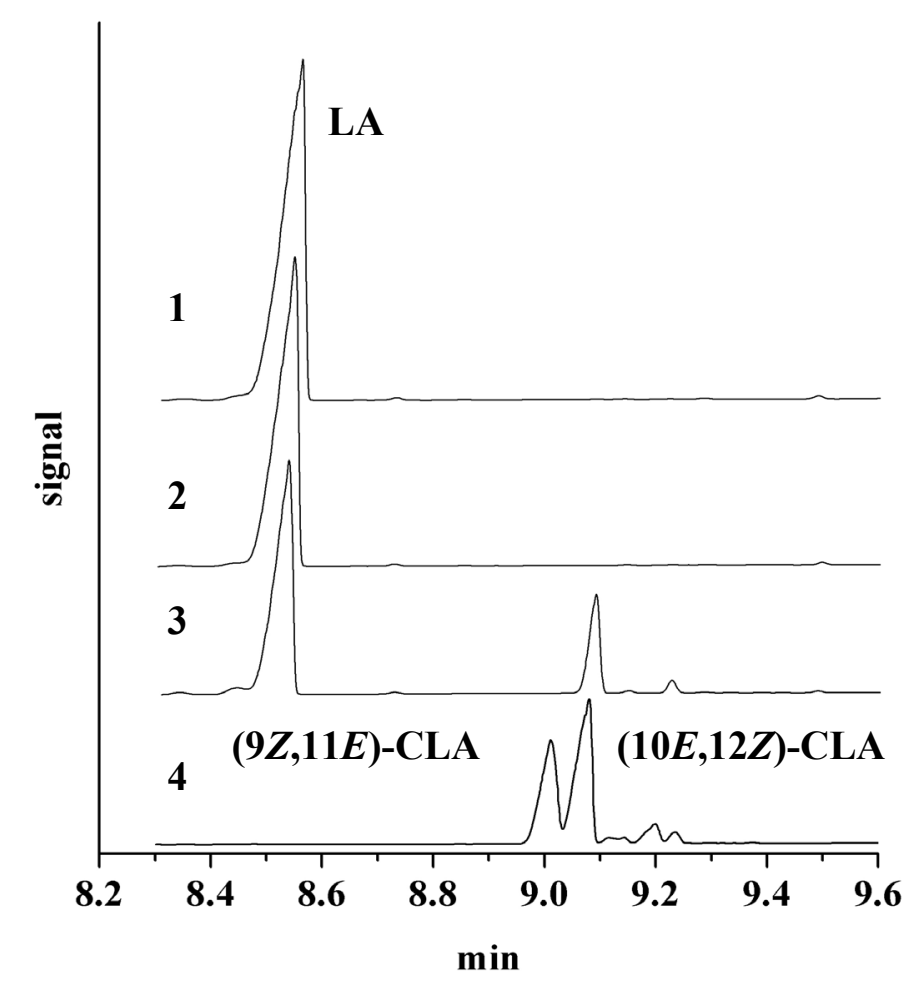

(b)

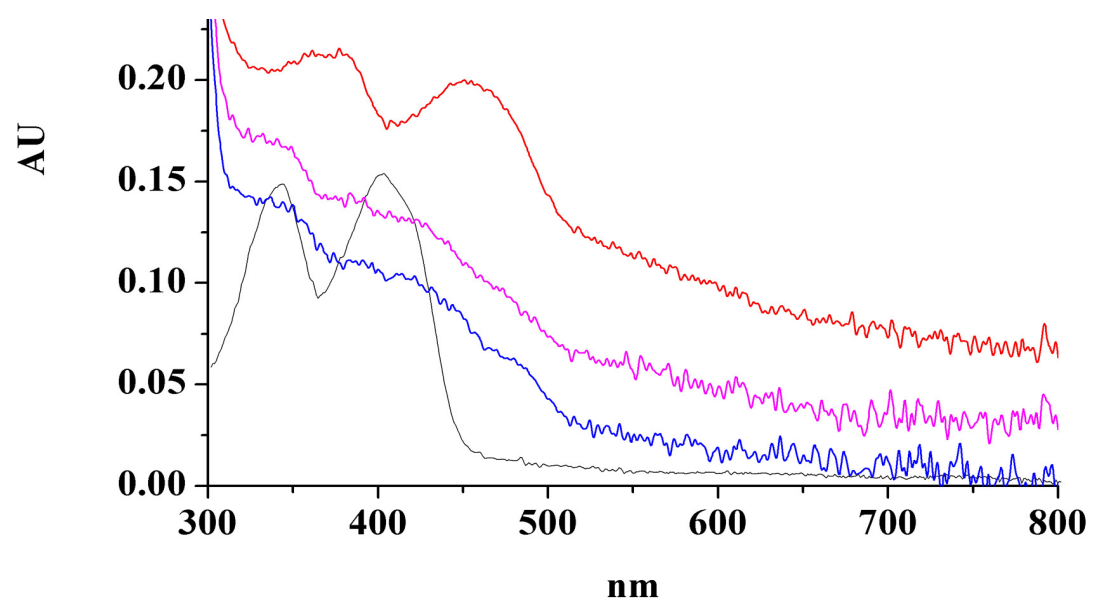

Fig. 26 Reconstitution of PAI with 5-deaza-5-carba-FAD.

(a) GC profiles of reaction products obtained after incubation of corresponding protein with LA are shown. 1 - apo PAI, 2 - apo-PAI + 5-deaza-5-carba-FAD, 3 - apo-PAI + FAD, 4 - CLA standard. Peaks of LA, (10E,12Z)-CLA and $(9 Z, 11 E)$-CLA are labelled. The apo-protein reconstituted with 5-deaza-5carba-FAD is not active, suggesting a radical mechanism. (b) UV-Vis spectra of PAI reconstituted with 5-deaza-5-carba-FAD. Black - $5 \mu \mathrm{M}$ 5-deaza-5-carba-FAD, blue - PAI + 5-deaza-5-carba-FAD + LA, mangenta - PAI + 5-deaza-5-carba-FAD, red - PAI + FAD. 
respectively, (Chapman 1999)), which can be insufficient for hydrogen removal from a non-activated aliphatic $\mathrm{C} 11$ of LA. In some cases the reverse reaction can still be catalyzed by the reduced 5-deaza-5-carba-FAD, but since PAI reaction is practically irreversible, this option could not be tested.

\subsection{FA hydratase from Streptococcus pyogenes (SPH)}

\subsubsection{Cloning, purification and spectral properties}

Based on sequence similarity with known PUFA double bond isomerases (Fig. 27, see also 3.4), an ORF from Streptococcus pyogenes annotated as "67 kDa myosine crossreactive antigen" (Kil et al. 1994) was predicted to have PUFA isomerase function. After overexpression of the isolated gene in E. coli, the recombinant protein turned out to catalyze hydration of double bonds in PUFAs, and therefore was renamed as $S$. pyogenes FA hydratase (SPH). SPH fused with an N-terminal 6xHis-tag was overproduced in $E$. coli BL21 Star, only the induction at $16{ }^{\circ} \mathrm{C}$ resulted in significant amounts of soluble protein. Affinity purification on $\mathrm{Ni}^{2+}$-affinity resin resulted in high yield of $6 \mathrm{xHis-} \mathrm{SPH}$ and the protein was about $90 \%$ pure as judged by Coomassie-stained gel (Fig. 28a, lane 1). The so obtained SPH was additionally purified by anion exchange (Fig. 28a, lane 2). The expected molecular weight (MW) of SPH is about $67 \mathrm{kDa}$ but according to SDSPAGE results it appeared to be about $60 \mathrm{kDa}$. Proteolysis and posttranslational modifications are most often reasons for the shifts of a protein MW on SDS-PAGE. The cleavage of $\mathrm{N}$-terminal signal peptide or other protein domain may be excluded since $\mathrm{SPH}$ was efficiently bound by $\mathrm{Ni}^{2+}$-affinity resin over the $6 \mathrm{xHis}$ tag. Additionally, the presence of tag in the purified protein was confirmed by immunoblotting with 6xHisspecific antibody (Fig. 28b, lane 5). To check the integrity of polypeptide chain and to detect possible modifications, SPH was subjected to mass-spectrometric analysis by MALDI-TOF mass spectrometry after proteolytic digestion with trypsin. The peptides identified within the amino acid sequence of SPH are shown in Supplem. Fig. 1. Total sequence coverage is about $25 \%$ and $\mathrm{C}$-terminal amino acids are detected. These data demonstrate the integrity of $\mathrm{N}$ - and C-termini of SPH protein. Therefore the most probable reason for the decreased MW is a posttranslational modification of so far unidentified origin. 

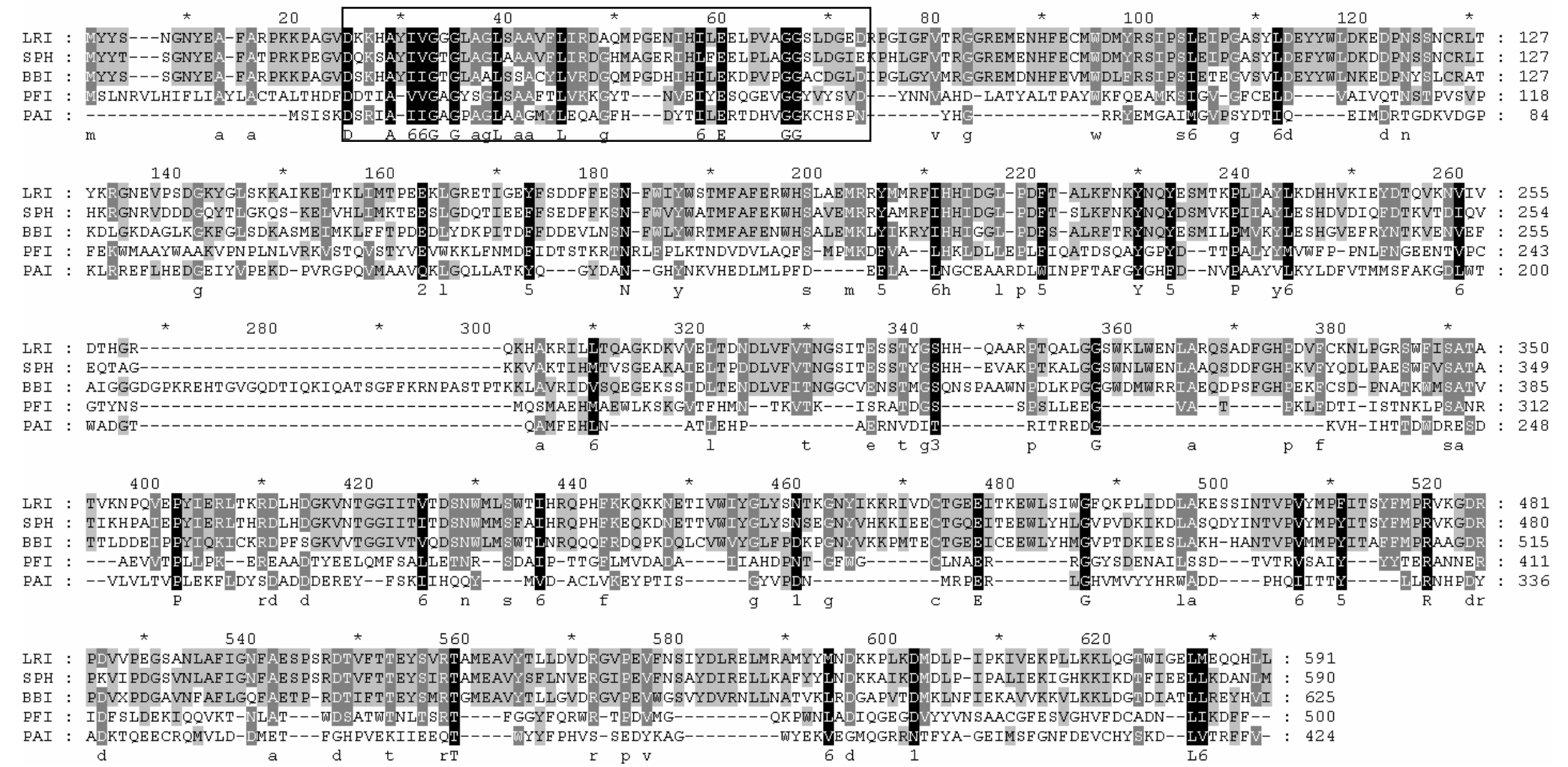

Fig. 27 Sequence alignment of putative PUFA double bond isomerases LRI, SPH and BBI with known isomerases PFI and PAI. The conserved flavinbinding domain is labeled by open box. 
(a)

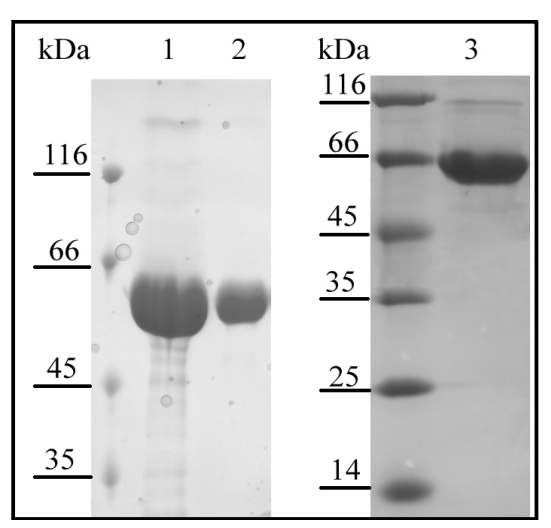

(c)

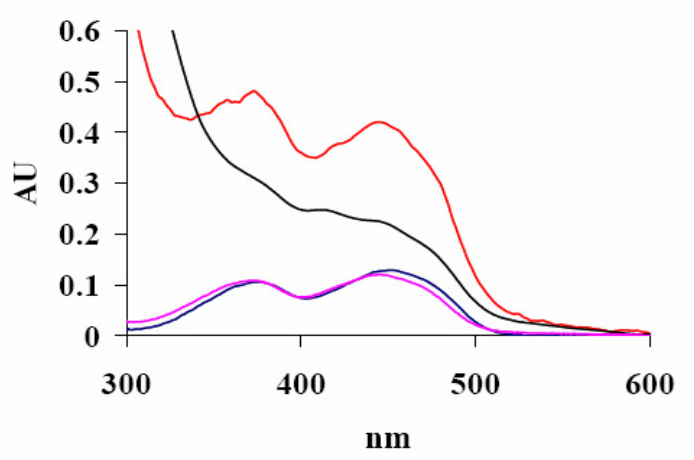

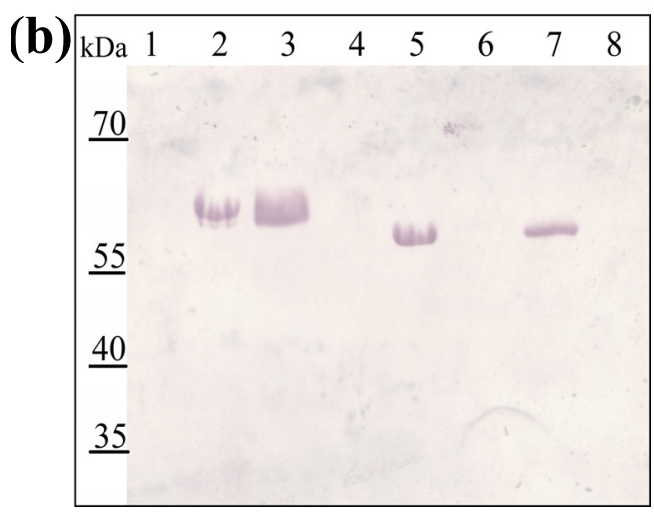

(d)

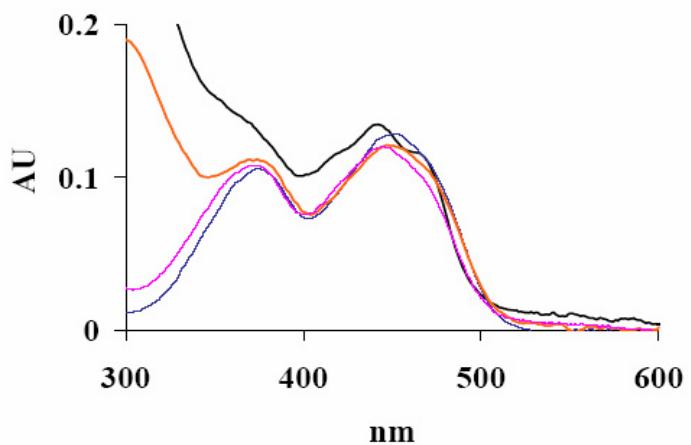

Fig. 28 Purification and properties of SPH and BBI.

(a) 1,2 - purified SPH after $\mathrm{Ni}^{2+}$-affinity column and ion exchange, respectively. Protein has apparent MW of 50-55 kDa (predicted MW $=67 \mathrm{kDa}$ ). 3 - purified BBI after $\mathrm{Ni}^{2+}$-affinity column. Protein has apparent MW of $66 \mathrm{kDa}$ (predicted MW $=74 \mathrm{kDa}$ ). (b) UV-Vis spectrum of SPH (black) and the supernatant after heat precipitation (red), showing flavin-like absorbance. For the reference spectra of $10 \mu \mathrm{M}$ FAD (blue) and FMN (magenta) are shown. (c) same as on (b) but for BBI protein. (d) Immunoblot with anti-His tag antibodies shows that N-terminal His-tag is intact in all recombinant proteins tested. 1 - cell lysate GST-BBI, 2 - purified BBI with N-term 6xHis tag, 3 - cell lysate LRI with N-term 6xHis tag, 4 - cell lysate LRI with C-term 6xHis tag, 5 - purified SPH with N-term 6xHis tag, 6 - cell lysate SPH no tags, 7 - cell lysate PFI with N-term 6xHis tag, 8 - cell lysate PFI with Cterm $6 \mathrm{xHis}$ tag.

Concentrated SPH solution had yellow color and UV-VIS spectrum of SPH shows maxima around 370 and $450 \mathrm{~nm}$ characteristic for flavin cofactors, as well as small additional peaks at 420 and $480 \mathrm{~nm}$ (Fig. 28c). The protein environment can strongly affect flavin absorption giving raise to shoulders and shifts in the spectrum compared to free flavins (Chapman 1999). Other cofactors associated with flavin, like metal ions or 
FeS clusters can also influence the spectrum. To test for the presence of flavin cofactors in SPH, the protein was heat-denatured and the spectrum of protein-free supernatant was recorded (Fig. 28c). In this case the spectrum contains only two maxima at 447 and 373 $\mathrm{nm}$ and closely resembles those of free FAD and FMN.

\subsubsection{SPH produces hydroxy FA species}

Initial activity assays with purified SPH showed that no CLA was formed from LA, instead, 10-HOE was detected by GC-MS as main reaction product (Fig. 29a). The peak of $\mathrm{m} / \mathrm{z}=273$ is a predominant feature of the spectrum and represents the cleavage of C10-C11 bond, resulting in a C10 fragment in which the 10-hydroxy group is modified by TMS. In order to characterize the enzymatic preferences towards double bond position and configuration, as well as headgroup polarity, a range of different FAs and lipids were assayed as SPH substrates. These data are summarized in Supplem. Table 5. Briefly, SPH shows the strict preference towards a free FA carboxy group and no complex lipids were used as substrates. It also requires (9Z)-double bond, $(9 E)$-, $(11 E)$ - and (11Z)-double bonds are not hydrated. Any combination of cis- and trans- double bonds as well as double bonds in front of $\Delta 9$ abolishes SPH activity. When an additional (12Z)-double bond was present in the substrate, di-hydroxy products are formed (Fig. 30a), but to the lesser extent than 10-HOE. The chain length of accepted FAs is limited to 16-18 carbons, C20 and C22-FAs are no substrates.

The origin of oxygen in the hydroxylated FA was established by performing the reaction in isotopically labeled water (Fig. 29b, c). In the mass spectrum of 10-HOE obtained after incubation in ${ }^{2} \mathrm{H}_{2} \mathrm{O}$ (Fig. 29b) the mass shift of +1 is evident for a C10 fragment described above. The mass of the ion resulting from the loss of O-TMS group is 295 , i.e. +1 a.m.u compared to corresponding signal in 10-HOE from regular water (Fig. 29a) and $\mathrm{H}_{2}{ }^{18} \mathrm{O}$ (Fig. 29c). This is clearly due to the incorporation of one ${ }^{2} \mathrm{H}$ atom at $\mathrm{C} 9$ position. Furthermore, after the reaction in $\mathrm{H}_{2}{ }^{18} \mathrm{O}$, the mass of $\mathrm{C} 10$ fragment increases by +2 a.m.u, indicating the addition of ${ }^{18} \mathrm{O}$ from water (Fig. 29c). These results demonstrate that no molecular oxygen is involved in the SPH reaction and the overall process can be described as enzymatic addition of one water molecule to the double bond. Therefore no redox equivalents are consumed and the role of flavin in the reaction remains unclear. 
(a)
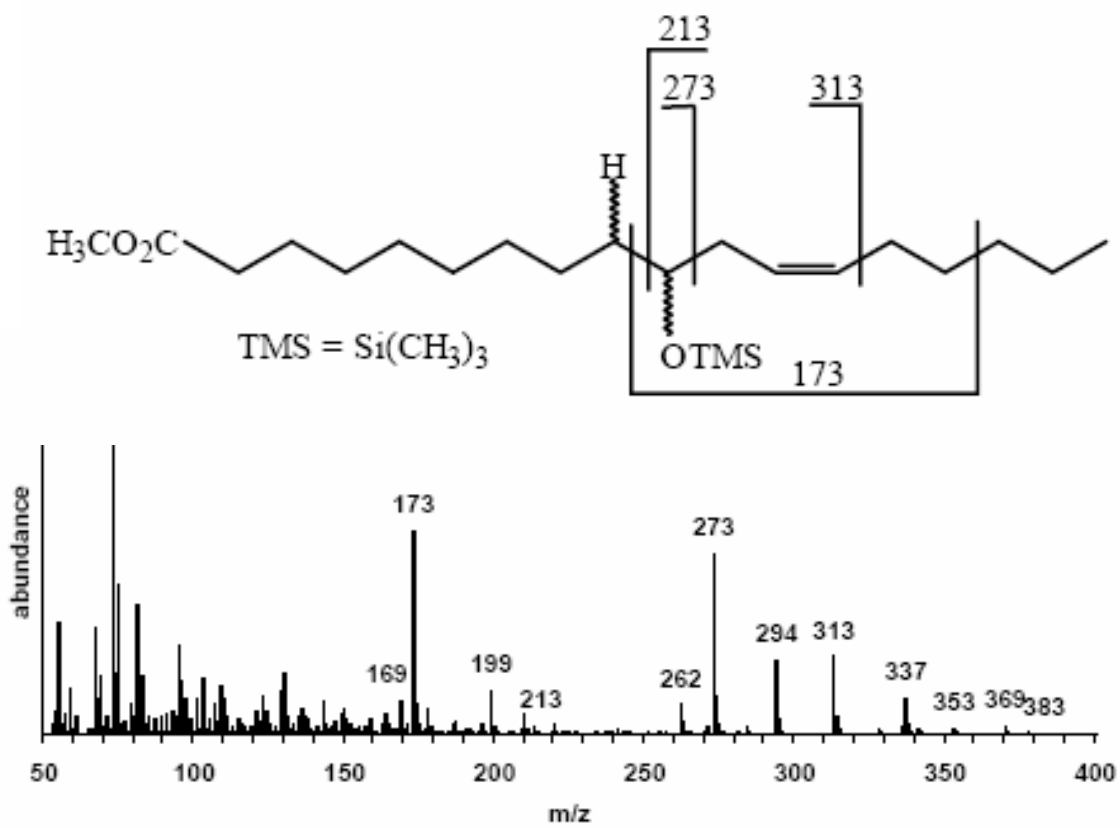

(b)

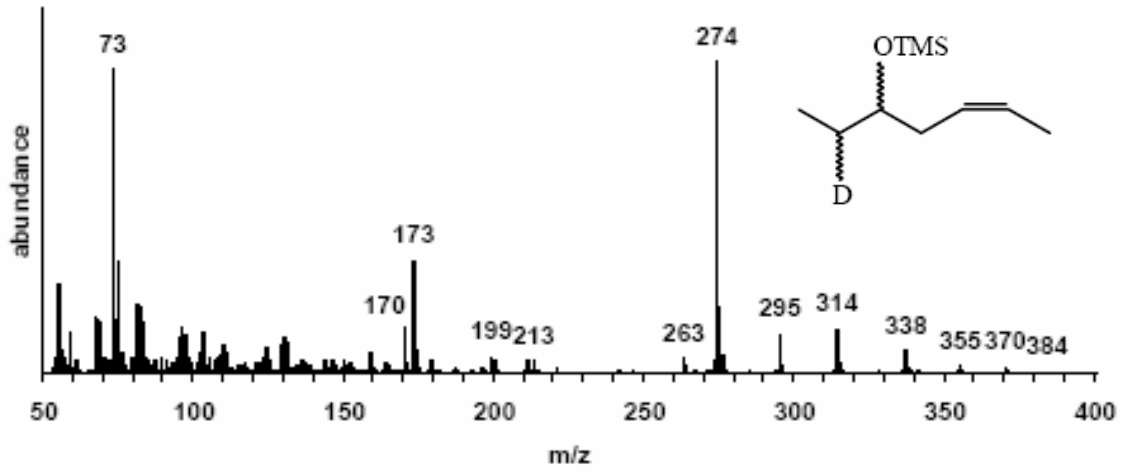

(c)

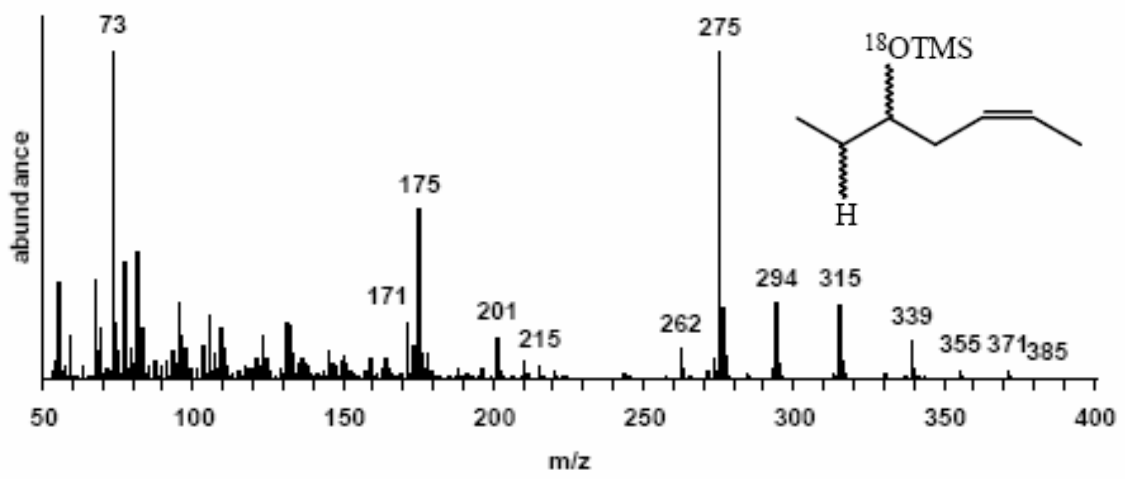

Fig. 29 Formation of 10-HOE by SPH.

(a) Mass spectrum of 10-HOE obtained after reaction of SPH with $\mathrm{LA}$ in $\mathrm{H}_{2} \mathrm{O}$, (b) in ${ }^{2} \mathrm{H}_{2} \mathrm{O}$, (c) in $\mathrm{H}_{2}{ }^{18} \mathrm{O}$. Structure of $10-\mathrm{HOE}$ and fragmentation pattern is shown on the inset. The mass of $\mathrm{C} 10$ fragment $(\mathrm{m} / \mathrm{z}=273)$ is changing due to the isotopic labeling. 
(a)
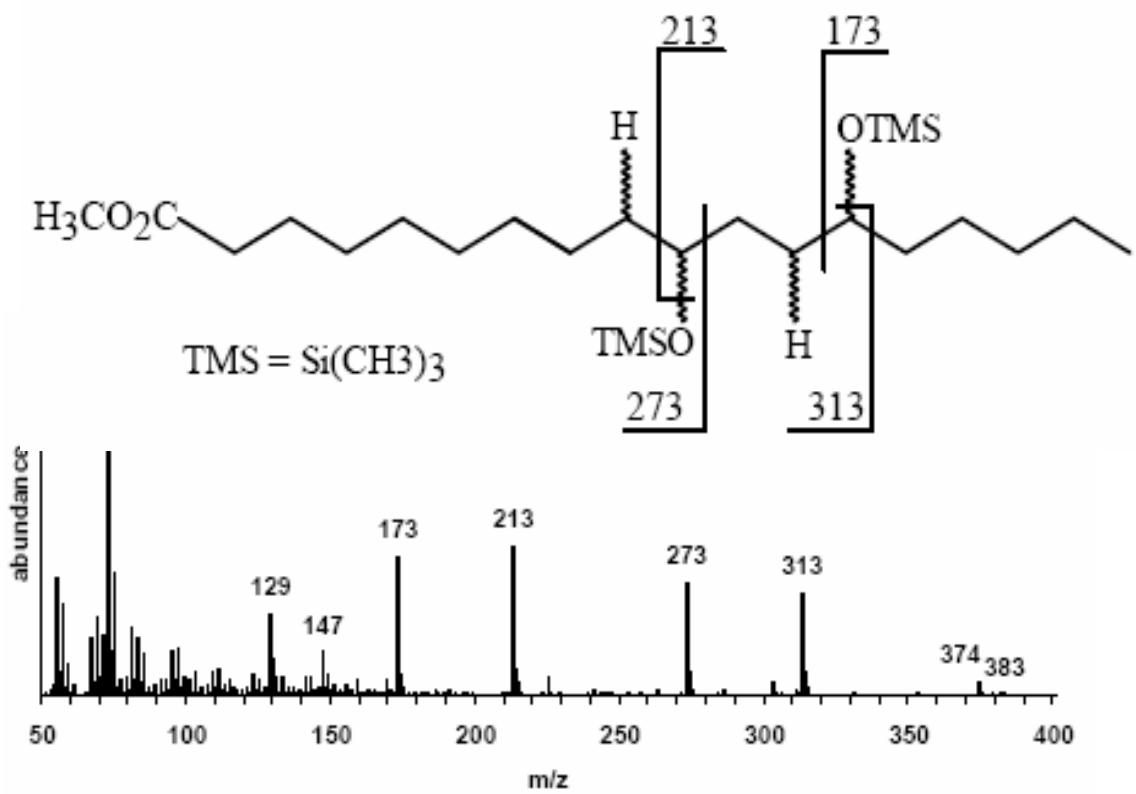

(b)

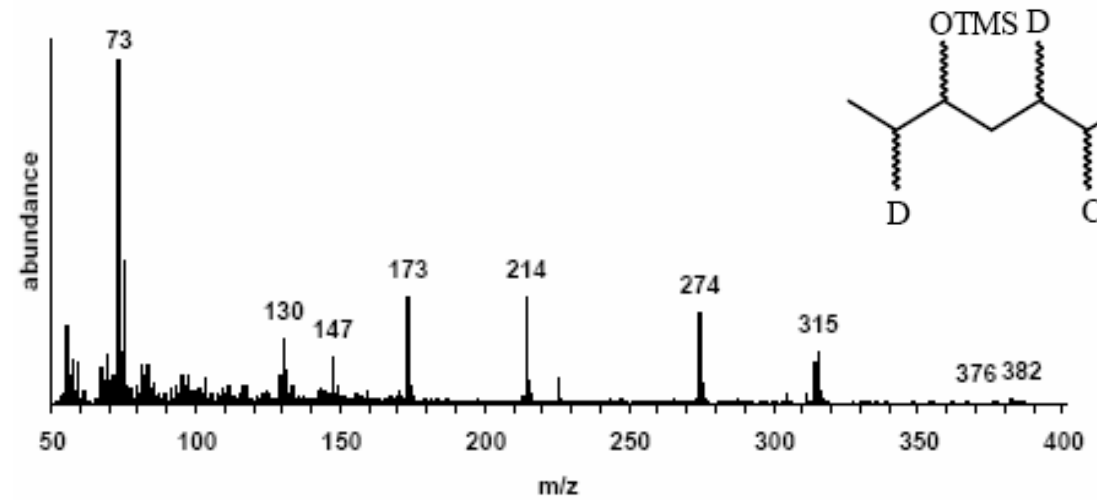

(c)

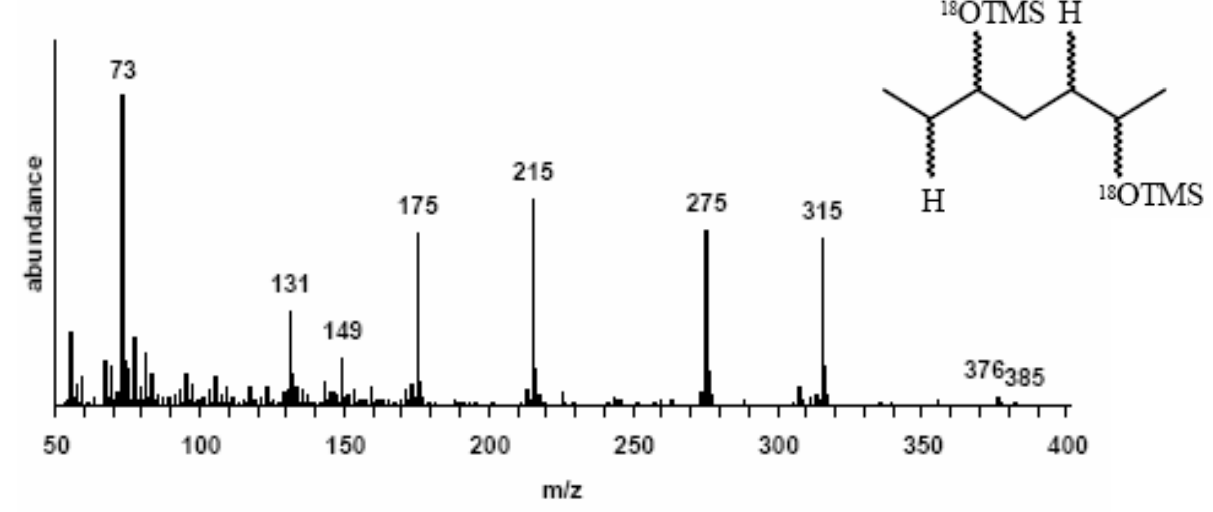

Fig. 30 Formation of 10,13-di-HO by SPH.

(a) Mass spectrum of 10,13-di-HO obtained after reaction of SPH with LA in $\mathrm{H}_{2} \mathrm{O}$, (b) in ${ }^{2} \mathrm{H}_{2} \mathrm{O}$, (c) in $\mathrm{H}_{2}{ }^{18} \mathrm{O}$. Structure of $10,13-\mathrm{di}-\mathrm{HO}$ and fragmentation pattern is shown on the inset. 
In a similar manner the structure of di-hydroxylated products can be analyzed; the example is 10,13-dihydroxy octadecanoic acid (10,13-di-HO) formed by SPH from LA. In Fig. 30 the mass spectra of 10,13-di-HO after reaction in $\mathrm{H}_{2} \mathrm{O}$ and ${ }^{2} \mathrm{H}_{2} \mathrm{O}$ are shown. Since all oxygen-containing fragments in the mass spectrum of 10,13-di-HO, resulted from incubation in $\mathrm{H}_{2}{ }^{18} \mathrm{O}$, are +2 a.m.u heavier, both double bonds are hydrated. The $\mathrm{pH}$ optimum of SPH is in the range of $\mathrm{pH}=6.8-7.0$ (Supplem. Fig. 3). No direct assay is available for the quantification of hydroxy groups in FAs, therefore the enzymatic activity was measured as the ratio of each product (10-HOE and 10,13-di-HO) to the internal standard AA, which is not hydrated by SPH (Supplem. Table 5). The formation of 10,13-di-HO seems to be most efficient at lower $\mathrm{pH}$ and decreases nearly linearly with $\mathrm{pH}$ increase, probably reflecting non-enzymatic hydration of the (12Z)-double bond after the first water molecule was already added by SPH to (9Z)-position.

To determine the stereoconfiguration at $\mathrm{C} 10$ atom of $10-\mathrm{HOE}$, reaction products were separated by straight phase HPLC, peaks collected and then analyzed by chiral-phase HPLC (data not shown). No clear resolution on the latter column was attained, and the lack of commercial standards did not permit to optimize the procedure.

Interestingly, other products with retention times higher than 10-HOE and 10,13-diHO were occasionally identified by GC-MS when LA was incubated with SPH (Fig. 32a). It turned out that the appearance of these products was associated with some batches of purified protein. The observation that repeated cycles of dilution-ultrafiltration lead to the loss of yellow color in the concentrated protein solution suggested that flavin binding is mediated by a small labile molecule/ion. It is known that often the phosphate group of nucleotide cofactors is coordinated to the protein core by divalent metal cations. Therefore, the colorless protein sample was tested for activity in the presence of FAD, 5carba-5-deaza-FAD or FMN alone and in combination with $10 \mu \mathrm{M}$ of the following metal cations: $\mathrm{Ca}^{2+}, \mathrm{Mg}^{2+}, \mathrm{Fe}^{3+}, \mathrm{Ni}^{2+}$. The apo-protein as well as the combination of FMN and 5-deaza-5-carba-FAD with any cation did not give any products, but strikingly the initially observed complex set of products (Fig. 32a) was fully reproducible when SPH was reconstituted with FAD and any cation. The mass spectra of the unidentified products are shown in Fig. 32b,c. These results suggest that SPH indeed contains FAD as cofactor and most probably its binding is dependent on one of the cations listed above. 
(a)

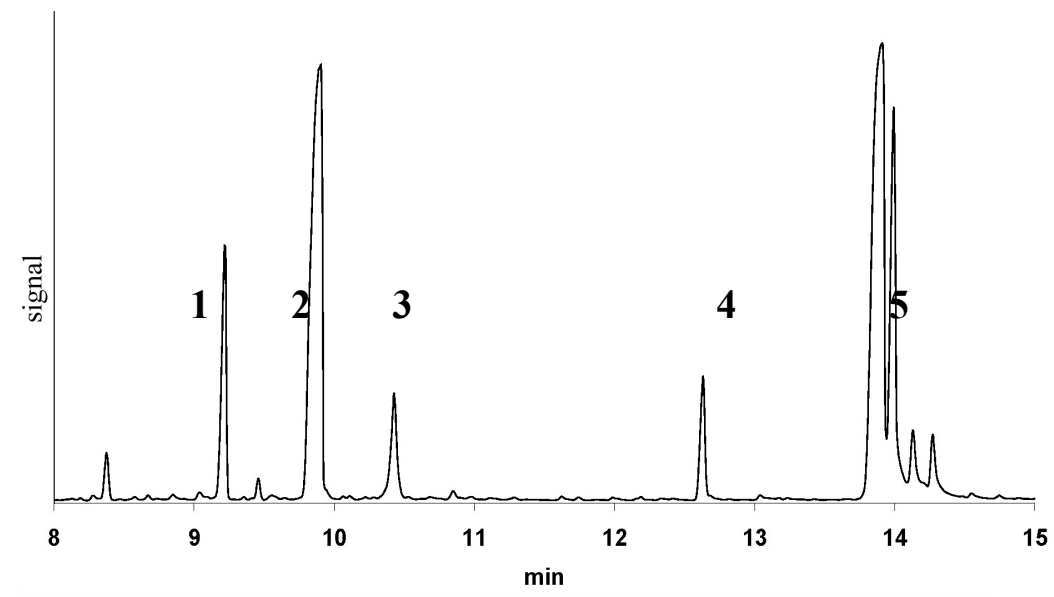

(b)

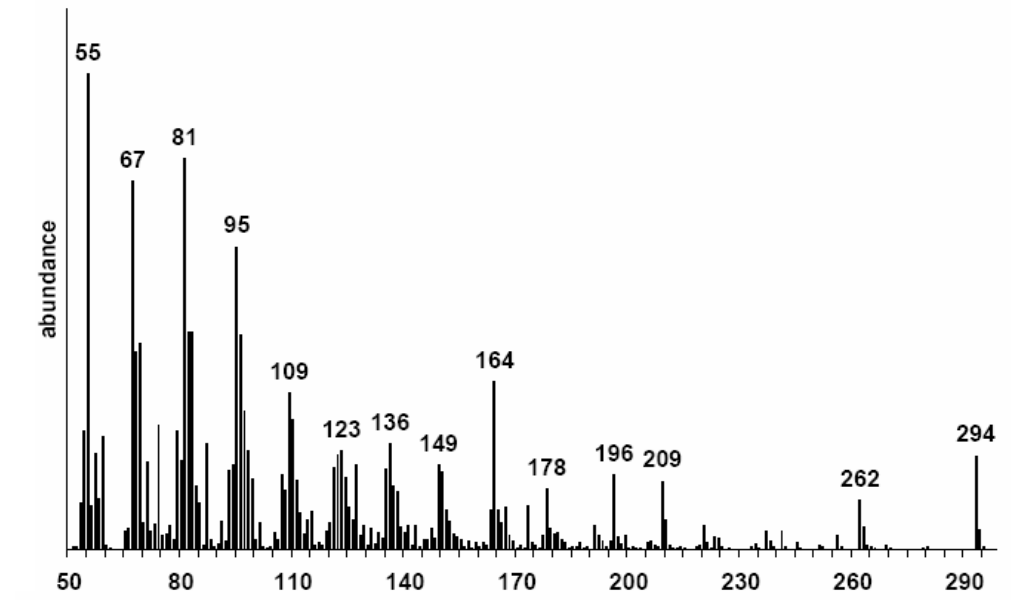

(c)

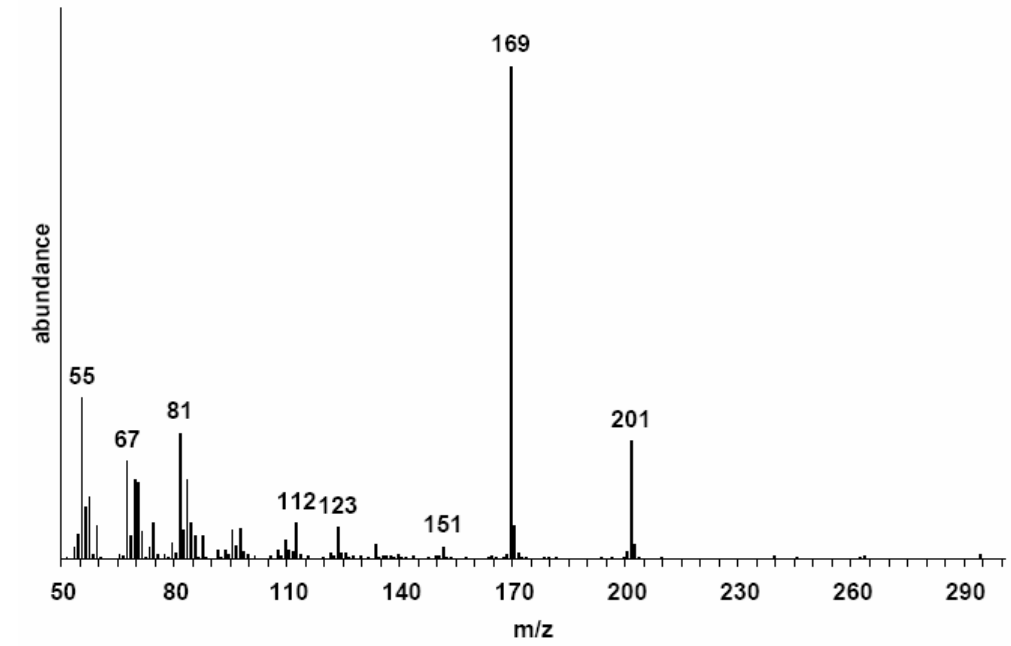

Fig. 32 Additional products formed by SPH.

(a) GC profile of SPH reaction products. 1 - LA, 2 - 10-HOE, 3 - 10,13-di-HO, 4 - extraction artifact, 5 and 6 - unidentified products. (b) Mass spectrum of 5. (c) Mass spectrum of 6 . The presence of two latter products was dependent on protein batch and depends upon presence of FAD and divalent cations. 


\section{4 $(9 Z, 11 E)$-CLA producing FA isomerases}

As already mentioned, to date PAI remains the only (10E,12Z)-CLA producing PUFA double bond isomerase studied in great details. Another broad class of PUFA double bond isomerases producing $(9 Z, 11 E)$-CLA is far more extensively investigated at the level of microbial LA metabolism, and includes the enzymes from gram-positive bacteria of Bifidobacterium, Lactobacillus, Clostridium and Butirivibrio species.

Surprisingly, these enzymes show very little amino acid sequence conservation with PAI and PFI (Fig. 27). Only N-terminal nucleotide binding motif seems to be conserved among all isomerases, suggesting that FAD might be the common cofactor employed for PUFA isomerization. In order to investigate reaction details of $(9 Z, 11 E)$-CLA producing isomerases, three genes, namely BBI, LRI and PFI were selected and cloned as N- or Cterminal fusion with $6 \mathrm{xHis}$ tag.

\subsubsection{Bifidobacterium breve isomerase (BBI)}

BBI was subcloned into pET28 vector as N-terminal 6xHis fusion (Suppl. Table 1), the protein overproduced in E. coli BL21 cells and purified on $\mathrm{Ni}^{2+}$-affinity column (Fig. 28a). The predicted MW for BBI is $74 \mathrm{kDa}$, however, it appears to be around $66 \mathrm{kDa}$ judged by SDS-PAGE. The integrity of polypeptide chain in the purified BBI protein was analyzed in similar way as described for SPH, i.e. the presence of N-terminal 6xHis-tag was confirmed by imunoblotting (Fig. 28b) and the C-terminal fragments were found in the mass spectra by MALDI-TOF mass spectrometry (Supplem. Fig. 2). Although around 30 amino acids from C-terminus of BBI were not found in the mass spectrum (due to the absence of trypsin recognition sites in this region), but this fragment is too small to account for the $10 \mathrm{kDa}$ difference in MW. It is likely that both SPH and BBI proteins undergo some modifications affecting their electrophoretic behavior. However, the type of this modification is yet to be established.

The FAD-binding motif is conserved in PUFA double bond isomerases described here (Fig. 27). Purified BBI protein had a yellow color and the absorption spectrum displays flavin-like maxima around 450 and $370 \mathrm{~nm}$ (Fig. 28d). The spectrum seems to contain contributions from other functional groups and/or cofactors (see 3.3.1) and a protein-depleted solution shows the clearer maxima close to the FAD absorption spectrum. Thus, it is likely that BBI also contains a non-covalently bound FAD as a 


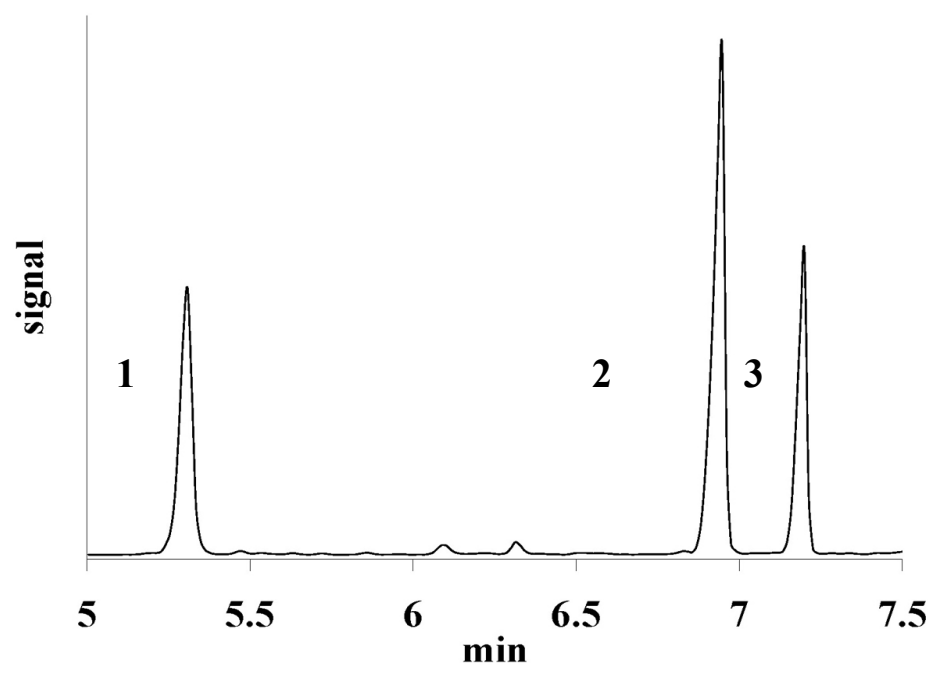

Fig. $33 \mathrm{BBI}$ is purified in complex with free FA.

The GC-MS profile of extract from purified concentrated BBI is shown. FAs were identified by mass spectra. 1 - hexadecanoic acid, 2 - stearic acid, 3 - oleic acid.

cofactor, implying a similar reaction mechanism as in case of PAI. Surprisingly, no CLA was detected after purified BBI was incubated with LA. Instead, small quantities of 10HOE were found in the GC-MS profiles of BBI reaction products, independent of protein amount added. A number of FAs was tested and no isomerase activity was found for any other substrate (Supplem. Table 6). Only OA and PA were hydrated to 10-hydroxy products in trace amounts (data not shown). Despite the fact that BBI was purified as soluble protein and could be concentrated to $10-20 \mathrm{mg} / \mathrm{ml}$, these data indicate that active site might be compromised. When $100 \mu \mathrm{L}$ of concentrated protein was treated according to a FA extraction protocol (see Methods) and the extract analyzed by GC-MS, three major peaks in the resulting chromatogram appeared, corresponding to PA, SA and OA (Fig. 33). No FAs were detected when the extract was transmethylated with sodium methoxide (data not shown), excluding the presence of lipid-derived FAs. Thus, during the purification procedure $\mathrm{BBI}$ retains tightly bound free FAs and this is the most likely reason for its compromised activity. Whether BBI accumulates these FAs during protein synthesis in E. coli, or this is a purification artifact remains unclear. In any case, the activity of BBI remains to be tested after FAs removal, e.g. by detergent treatment or oncolumn refolding of the protein. 
LRI and PFI proteins turned out to be non-soluble in form of $\mathrm{N}$-terminal $6 \mathrm{xHis}$ or GST-fusions and therefore no activity test could be performed. The presence of a His tag was confirmed by immunoblotting (Fig. 28b) and the issue of solubility can be thus addressed by on-column refolding in the presence of stabilizing agents and cofactors. 


\section{Discussion}

\subsection{PAI structure and reaction mechanism}

Despite detailed biochemical studies on BFI and PFI PUFA double bond isomerases, including substrate specificity, product identities and hydrogen transfer during the reaction, the complete understanding of enzymatic mechanism was impeded by the lack of structural data. The main goal of this work was to determine the three-dimensional structure of PAI and to elucidate chemical reactions in the active site, underlying the formation of $(10 E, 12 Z)$-CLA from LA. Six structures of PAI in two space groups were determined by X-ray diffraction, including the structures of PAI-(10E,12Z)-CLA and PAI-(11E,13E,15Z)-CLnA complexes; these data provided detailed insight in the active site geometry and reaction mechanism. There were no major differences between six structures, except for active site and substrate binding tunnel, excluding possible crystallization artifacts.

PAI is a monomeric protein composed of three domains. In line with the preliminary spectroscopic data, one FAD molecule per monomer was found in the structure.

Surprisingly, PAI does not structurally resemble any FA-processing enzymes, rather it displays similar fold with unrelated flavoproteins PAO and UDP-galactopyranose mutase, mainly due to the conserved Rossman fold of FAD-binding domains in all three enzymes. Interestingly, the substrate of PAO is spermine, a 16-atoms long acylated polyamine possessing an acyl headgroup and long tail made of aliphatic diamine units (Huang et al. 2005) and thus distantly resembling the shape of FA molecule. Spermine binds in a tunnel between two PAO domains and C11 atom (counting from acyl headgroup) is positioned in front of the N5 atom of FAD for subsequent oxidation. The similar folds of PAI and PAO most likely evolved driven by analogous substrate shapes and requirement for $\mathrm{FAD}$ cofactor.

It should be noted, that a search in the public databases at the protein sequence level did not reveal any hits with significant similarity to PAI except domain 1 . Thus, for the present moment PAI remains a single member of CLA producing PUFA double bond isomerases studied at structural level.

The quality of electron density defining FAD molecule allowed unambiguously model the isoalloxasine ring of the latter into so called "butterfly" conformation, meaning 
the deviation from planarity in the oxidized aromatic isoalloxazine ring along the N5-N10 axis (Fig. 16c). It is generally assumed that distortions imposed on the aromatic part of FAD by the protein account for higher redox potentials in flavoenzymes compared to free FAD. For example, this type of conformational distortion was studied in great detail for cholesterol oxidase (Lario et al. 2003), where a single amino acid mutation (His121Ala) resulted in a planar geometry of FAD and decreased the redox potential by $100 \mathrm{mV}$ (Lim et al. 2006). Similar FAD geometries have been observed in monoamine oxidase B (Binda et al. 2003), glucose oxidase (Wohlfahrt et al. 1999) and lipoamide dehydrogenase (Mattevi et al. 1991).

Once crystallization of a protein was achieved, a very common approach in structurebased studies of enzymatic reactions mechanisms is to use transient state analogs or mechanism-dependent inhibitors, revealing the geometry of activated enzyme-substrate complex. Ideally, the structures of enzyme-substrate, activated enzyme-substrate and enzyme-product complexes should be available. In case of PAI, the nature of the substrate poses a challenge to such an approach, since it is generally difficult to modify middle part of FAs aliphatic chain by the methods of organic synthesis. Moreover, the geometric differences between LA and CLA are subtle, and the lack of reactive groups eliminates the possibility of dead-end inhibitors. LA fluorinated at $\mathrm{C} 11$ would be the best substrate-mimicking analog, but this substance is not available commercially.

LA was modeled to the additional electron density around FAD after cocrystallization of PAI with LA (Fig. 18a). The GC analysis, however, confirmed that $(10 E, 12 Z)-C L A$ rather than LA was bound to PAI in the crystals. Given the limited space for the design of chemical analogs, the structure of the PAI-(10E,12Z)-CLA complex nevertheless provided very good approximation of the enzyme-substrate complex. It revealed the determinants of PAI selectivity towards free C18-C20 FAs as well as possible reaction mechanism.

Hitherto, the only crystal structure of an enzyme bound to free PUFA is PGHS-1 (PDB entries 1IGX, 1IGZ, 1FE2) (Malkowski et al. 2001). In this complex LA adopts an extended form with carboxylic group making polar contacts to $\operatorname{Arg} 120$ and Tyr355, this binding mode is different from PAI. The basis for the free carboxylate recognition in PAI is the interaction of "lock" residues Arg88 and Phe193 upon substrate binding. 
Comparison of free PAI, PAI-PEG400 and PAI-(10E,12Z)-CLA structures showed the flexibility of Arg88 and Phe193 side chains (Fig. 21c). It is therefore likely that binding of LA triggers the closed conformation of the lock. Moreover, the positive potential on the PAI surface generated by Lys85, Arg87, Lys102, and Lys195 revealed a site for initial recognition of substrate carboxylate groups. The gating mechanism of substrate entry in PAI has two important consequences: the distance between the lock and N5 of FAD is restricted to eleven carbons and the access of solvent and oxygen to the active site is blocked, protecting the reactive intermediates from side-reactions. The PUFA double bond isomerases BFI and PFI prefer a carboxylic group over esterified substrates as well, and PFI also contains a flavin cofactor, likely FAD (Zheng et al. 2002). Thus, the ArgPhe lock or a similar interaction between gating amino acids might be a general feature of FAD-containing PUFA double bond isomerases.

The strict preference of PAI towards free FAs, which are rather scarce in eukaryoric cell, rendered inefficient the attempts to generate transgenic plants producing CLAcontaining oils. For example, when PAI was expressed heterologously in seeds of Nicotiana tabacum, the level of $(10 E, 12 Z)$-CLA accumulation in the seed oil did not exceed $0.4 \%$, while substantial amounts of $(10 E, 12 Z)$-CLA were detected in the free FA pool (Hornung et al. 2005). Similar results were reported for transgenic rice plants expressing PAI gene in the grains; the highest proportion of $(10 E, 12 Z)$-CLA in these seeds was $1.3 \%$ of total FA and CLA was accumulating mainly in the TAG fraction (Kohno-Murase et al. 2006).

In order to improve PAI activity on esterified FAs, the role of Arg88 and Phe193 in the substrate binding was investigated by site-directed mutagenesis. The values of $K_{m}$ for LA were increased 13-, 7- and 5-fold for Arg88Ser, Phe193Ala and the double mutant, respectively, indicating less efficient LA binding. However, $\mathrm{K}_{\mathrm{m}}$ for Ser88Ala193 mutant does not approximate the sum of $\mathrm{K}_{\mathrm{m}}$ values of the single mutants, as would be expected. In fact, $K_{m}$ value for the double mutant is lower than for each of the single mutants, which may be explained as following. In the double mutant the lock is permanently "open", since both gating residues have small sidechains eliminating a steric hindrance at the entry to the active site. Moreover, the lack of positively charged Arg88 leads to suboptimal hydrogen bonding of LA carboxylate group, and therefore $\mathrm{k}_{1} / \mathrm{k}_{2}$ value is 
decreased (Scheme 1). When only one of the gating residues is missing, the remaining one sterically blocks the entrance, unable to adopt a substrate-driven "closed" conformation in the absence of an interaction partner. Thus, the $\mathrm{k}_{1} / \mathrm{k}_{2}$ value increases even more compared to the double mutant. Remarkably, the Arg88S mutant has the highest $\mathrm{K}_{\mathrm{m}}$, underscoring the importance of ionic interaction between $\operatorname{Arg} 88$ and free carboxylate, so that exchange of Arg88 to unpolar Ala lowers PAI activity to the detection limits. As discussed below, the proper substrate positioning is extremely important for the efficient hydrogen transfer, and this effect is reflected in decreased $\mathrm{V}_{\max }$ values for all mutant proteins.

Unlike predicted, mutations of Arg88 and Phe193 do not significantly change PAI activity towards esterified FAs (Suppl. Table 4). A clear difference is observed only for LA methyl ester, which was converted to (10E,12Z)-CLA by the Arg88Ser mutant 4-fold more efficiently than by wt PAI, but overall only about $5 \%$ of the added substrate was isomerized. Taking into account the geometry of the substrate-binding tunnel, which provides narrow space for a single aliphatic chain (Fig. 21a), it is unlikely that a simple knock-out of gating residues or neighboring surface residues will improve the binding of complex lipids. Search at Protein Data Bank yielded three protein structures with the bound lipid substrate, namely human phosphatidylinositol (PI) transfer protein alpha bound to PI (PDB ID 1UW5, (Tilley et al. 2004)), human antigen-presenting glycoprotein CD1b in complex with PI (PDB ID 1GZQ, (Gadola et al. 2002)) and human phosphatidylcholine (PC) transfer protein in complex with dilinoleoyl-PC or palmitoyllinoleoyl-PC (PDB ID 1LN1, (Roderick et al. 2002)). In all cases the bent FA tails of the lipids are buried inside the protein cavities, which are formed mostly by extended $\beta$ sheets and topped with one or more $\alpha$-helices. Notably, the shape and volume of these cavities is completely different from the substrate binding tunnels found in PAI and human PGHS-1, highlighting conceptual differences in the architecture of free FAs and lipid-binding proteins. Despite this, the observed formation of significant amounts of (10E,12Z)-CLA from linoleyl-CoA thioester leaves the room for subcellular targeting approach, e.g. PAI may be localized to the cytosolic side of ER or plastidial membranes, which are cellular compartments with relatively high acyl-CoA levels. 
Stretching from the PAI surface towards the active site, the aliphatic tail of CLA andopts an U-shaped conformation, and it is contacted by hydrophobic amino acids and isoalloxazine ring of FAD (Figs. 18a, 19b). Although FAD is tightly bound to PAI and does not dissociate upon action of chaotropic salts or at low $\mathrm{pH}$, a shift of the isoalloxazine moiety was observed upon FA binding, indicating inherent flexibility of the cofactor part involved in the reaction. This might be important both for proper FA positioning in the active site and catalytic efficiency. The close distance between $\mathrm{C} 11$ of the FA and N5 of FAD in the PAI-(10E,12Z)-CLA complex strongly suggests that FAD acts as a hydrogen-abstracting electrophil. As no cysteine, tryptophan or acidic residues are present in the active site, non-FAD redox and proton abstraction processes can be excluded. The only major difference between free PAI and the complex is the rotation of the Phe168 side chain, which becomes co-planar with atoms C9-C13 of (10E,12Z)-CLA (Fig. 19b). Thus, assuming a similar spatial arrangement, the pentadienyl system of LA should be stacked between two aromatic rings in the enzyme-substrate complex, in which the $\mathrm{C} 11-\mathrm{H}$ bond would be aligned with the LUMO of FAD, enabling hydrogen transfer to N5. The observed binding mode immediately suggests a two-step reaction mechanism based on transient oxidation of LA by FAD. The first step in the catalytic sequence is the abstraction of pro- $R$ hydrogen from $\mathrm{C} 11$ by N5 of FAD (Fig. 22a). The intermediate carbocation or radical is stabilized by the interaction with Phe168 and flavin aromatic systems and the isomerization of C9-C10 double bond occurs driven by resonance stabilization of conjugated diene (estimated to be about $-16.7 \mathrm{kJM}^{-1}$ in 1,3-butanediene). The regiospecific hydrogen transfer from $\mathrm{N} 5$ to the pro- $R$ position of $\mathrm{C} 9$ can then proceed either in a concerted manner with the double bond shift or as a discrete step at the last stage of reaction, completing the catalytic cycle (Fig. 22b). The preferred direction of allylic shift is explained by the relative positions of Phe168 and the intermediate. Because the benzene ring of Phe168 is closer to C9 rather than C13 atom of LA, the mesomer with a partial positive charge on $\mathrm{C} 9$ will stabilized, and $(10 E, 12 Z)$-diene will be fixed by hydrogen transfer from FAD. Mutations of Phe168 to smaller hydrophobic residues impaired FAD binding and PAI reactivity was decreased to undetectable levels, underscoring the crucial role of this residue for the catalysis. 
Two important considerations concerning the reaction mechanism arise from the structural model of PAI reaction. First, the dissociation energy of a C-H bond in the absence of vicinal activating groups is high, implying either the involvement of a redox group with high midpoint potential or hydrogen tunneling in the active site. And second, the possibilities of homolytic and heterolytic bond cleavage should be evaluated, given that flavins serve equally well as radical and hydride acceptors (Fig. 12).

In terms of classical transition state theory, enzymes increase reaction rates by orders of magnitude creating an active site environment complementary to the shape of substrate transition state. For example, the stabilization of enolate intermediates by oxoanione hole in ECI and KSI enzymes was described in the introduction. In general, it is almost impossible to estimate separately the contributions from geometric complementarity and electrostatic interactions to the overall efficiency of given enzyme. Reactions involving hydrogen transfer can be classified according to the electronic state of hydrogen as proton, hydride or hydrogen atom transfer. Proton transfer is widely employed in enzymatic acid-base catalysis, and hydride is usually removed by cofactor-dependent oxidases. The unique feature of enzymatic hydrogen atom transfer is the high barrier for homolytic cleavage of $\mathrm{C}-\mathrm{H}$ bond, which should be overcome at mild conditions of enzymatic reactions. In this case electrostatic stabilization of the transition state is difficult, as the charge distribution in ground and activated state does not vary significantly (Klinman 2006). Several enzymes were shown to solve this problem by hydrogen tunneling, e.g. LOX, galactose oxidase and methane monooxygenase (Kohen \& Klinman 1999). Hydrogen tunneling coupled to thermal induced enzyme dynamic was also demonstrated in flavin- and quinone-dependent dehydrogenases (Sutcliffe \& Scrutton 2002) and in the flavoproteins morphinone reductase and pentaerythritol tetranitrate reductase (Basran et al. 2003). Proper positioning of the reactants, minimizing the distance between the active groups is one of the basic principles in enzymatic catalysis, it is also of paramount importance for tunneling efficiency as demonstrated by mutagenesis studies on alcohol dehydrogenase (Bahnson et al. 1997). In the alcohol dehydrogenase structure, hydrogen donor and acceptor atoms (C2 of trifluoroethanol and C4 of NAD) are positioned $3.2 \AA$ apart from each other, which is sufficient for hydrogen tunneling. The corresponding distance between C11 of LA and 

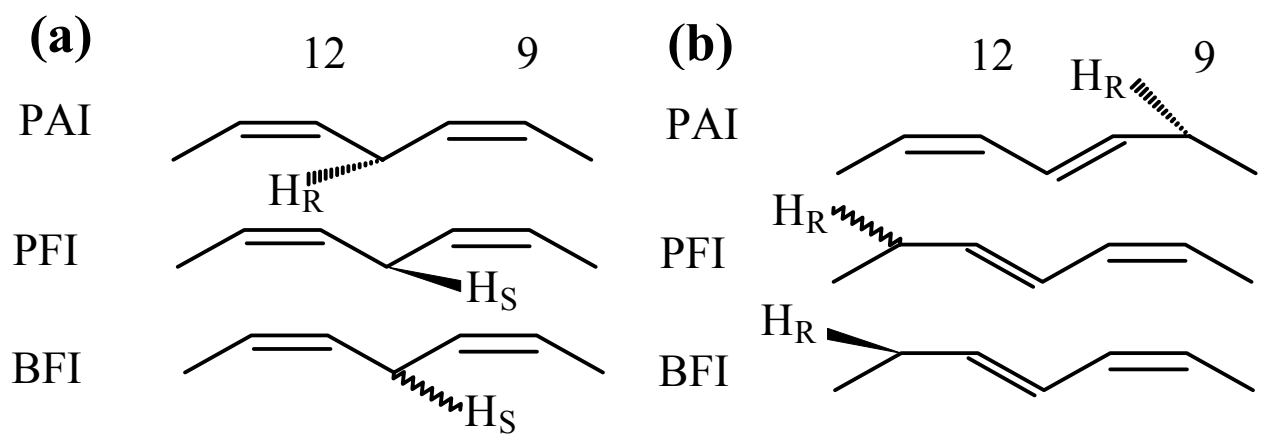

Fig. 34 Stereochemistry of hydrogen transfer in PUFA isomerases.

(a) Stereoconfiguration of hydrogen abstraction. Data are available for PAI and PFI (solid lines), but not for BFI (waved line). (b) The same for stereoconfiguration of hydrogen addition, which was directly demonstrated only for BFI, and predicted from structural model for PAI.

N5 of FAD in PAI-(10E,12Z)-CLA complex is also $3.2 \AA$, indicating that the efficiency of catalysis may relay on hydrogen tunneling as well. This possibility as well as the stereochemistry of hydrogen transfer was addressed by studies with isotopically labeled LA as described below.

C11 was confirmed as hydrogen abstraction site by FAD using 11-dideuterio-LA as PAI substrate (Fig. 23c). In a similar manner deuterium transfer in (11S)-deuterio-LA was traced, and since the label remained at initial C11 position, the (11R)-hydrogen must be abstracted by PAI, in full agreement with the structural model. This finding creates an important link between (10E,12Z)-CLA and $(9 Z, 11 E)$-CLA producing PUFA double bond isomerases, because both PFI and BFI enzymes were shown to remove hydrogen at C11 of $\gamma$-LnA and LA, respectively. Unfortunately, the data for hydrogen transfer by PAI, PFI and BFI are not fully complete in terms of stereochemistry (Fig. 33). In BFI the site of hydrogen abstraction was suggested to be C11, based on KIE measurements, and the stereoconfiguration of hydrogen addition to $\mathrm{C} 13$ was shown to be pro- $R$ (Kepler et al. 1971). Pro- $R$ and pro- $S$ hydrogen atoms are abstracted by PAI and PFI respectively, but the configuration of hydrogen addition was not confirmed (present work and (Wise et al. 1994)). However, the orientation of (10E,12Z)-CLA in the active site of PAI strongly suggests that hydrogen is transferred to $\mathrm{C} 9$ in pro- $R$ configuration. In contrast to PAI, both PFI and BFI incorporate solvent-derived hydrogen in the conjugated products. Assuming a FAD-dependent catalysis, the direct protonation by solvent or acidic amino 
acids is extremely unlikely, because the recycling of reduced FAD would imply additional redox cofactors, which were not required by either enzyme. Moreover, unpolar active site environment and water exclusion upon substrate binding might be also employed by BFI and PFI taking into account the similar substrate preferences. A plausible explanation would be the rapid proton exchange between reduced $\mathrm{N}-\mathrm{H}$ group of FAD and solvent, occurring from the antarfacial side of isoalloxazine ring relative to FA binding site.

Important information about atomic motions during the reaction can be gained from KIE studies. The measurement of primary KIE for PAI reaction was not performed since $(11 R)$-deuterio-LA was not available, therefore indirect data were acquired by comparison of kinetic parameters for 11-dideuterio-LA and (11S)-deuterio-LA. KIE values for these substrates were 5.3 and 1.3, respectively, compared to non-labeled LA (Table 2). $K_{m}$ value for 11-dideuterio-LA was twice less than for LA, proving that the breakage of $\mathrm{C}-\mathrm{H}$ bond is indeed the rate-limiting step in the overall reaction. As already mentioned, the reaction equilibrium is strongly shifted to $(10 E, 12 Z)$-CLA formation due to the negative Gibbs energy of conjugated diene formation. Therefore, isomerization of LA to $(10 E, 12 Z)-C L A$ can be depicted by a simple Michaelis-Menten kinetic scheme for irreversible reactions (Scheme 1). Hence, the reason of pronounced $\mathrm{K}_{\mathrm{m}}$ drop caused by deuteration at $\mathrm{C} 11$ is lower $\mathrm{k}_{3}$ value. For simplicity, this constant includes the rates of $\mathrm{C}$ $\mathrm{H}$ bond cleavage, pentadienyl isomerization, transfer of hydrogen to $\mathrm{C} 9$ and product dissociation. But in fact, the decrease in $\mathrm{k}_{3}$ reflects the slower hydrogen transfer steps (Fig. 22), which can be further discriminated by stopped-flow anaerobic measurements with monitoring of flavin semiquinone formation and decay. Hydrogen tunneling in enzymatic reactions can be demonstrated in two most common ways: comparing the KIE between three hydrogen isotopes - H, D and T or, alternatively, studying the temperature dependence of KIE for H and D (Klinman 2006). These expirements were not conducted in the present work because of the lack of isotopically labeled substrates. Nevertheless, KIE for 11-dideutero-LA is indicative of possible tunneling effects (the values of $\mathrm{k}_{\mathrm{H}} / \mathrm{k}_{\mathrm{D}}=$ 4-10 were reported in several systems (Klinman 2006)).

Cleavage of $\mathrm{C}-\mathrm{H}$ bond by flavoenzymes occurs in many biochemical reactions, for example flavin-containing dehydrogenases and acyl-CoA oxidases are central to the FA 
$\beta$-oxidation pathway (Kim \& Miura 2004, Ghisla \& Thorpe 2004). A number of flavoenzymes catalyzing reaction with no net redox change is known, and for most of them the direct involvement of the flavin in the catalytic events was demonstrated (Bornemann 2002). Interestingly, PAI fold resembles one of such enzymes - UDPgalactopyranose mutase, likely operating via a FAD radical (Sanders et al. 2001, Huang et al. 2003). Three dimensional structures of flavin-dependent isomerases IDI-2 and 4BUDH are known, and they are structurally unrelated to PAI, in both cases the radical abstraction of a non-activated hydrogen atom by flavin was demonstrated (Kittleman et al. 2007, Buckel \& Golding 2006). All these isomerases, including PAI, originate from anaerobic bacteria, in which a wide variety of radical reactions evolved in these microorganisms due to the absence of reactive molecular oxygen (Buckel \& Golding 2006).

FAD radical formation was observed upon anaerobic reduction of PAI (Fig. 25). The increase of absorbance at $600 \mathrm{~nm}$ suggested that a neutral flavin semiquinone was formed during the reduction (Chapman 1999). Anaerobic addition of LA to oxidized PAI perturbed the spectrum, but flavin radical maximum was obscured by a stronger peak around $550 \mathrm{~nm}$, probably reflecting a charge-transfer complex formation between stacked $\pi$-systems of Phe168, (10E,12Z)-CLA and FAD (Fig. 25a). Apart from spectrophotometric monitoring, the formation of flavin radicals can be demonstrated by a number of different techniques, e.g. NMR- and EPR-spectroscopy, redox titration of the enzyme in question and reconstitution of the apo-enzyme with synthetic flavin analogs, possessing variable propensities towards stable radical formation (Chapman 1999). In the latter case, 5-deaza-5-carba-FAD was introduced as a useful substance for probing radical mechanisms in flavoenzyme catalysis (Edmondson et al. 1972). N-centered radicals are more stable than $\mathrm{C}$-centered analogs and therefore incorporation of 5-deaza-5-carba-FAD usually leads to inactivation of radical-forming flavoproteins. PAI reconstituted with 5deaza-5-carba-FAD did not catalyze the LA isomerization, in contrast to the control sample reconstituted with FAD (Fig. 26), supporting the involvement of a FAD radical in the reaction. However, while retained enzymatic reactivity with 5-deaza-5-carba-FAD is usually regarded as a confirmation of hydride-transfer mechanism, independent methods are still required to prove radical formation once inactivation by this FAD analog was 
demonstrated. To this end, the EPR- and redox-measurements remain to be done with free PAI and the enzyme-substrate complex.

\subsection{Putative bifunctional FA hydratases-isomerases}

Acquired results about PAI structure and mechanism prompt to re-examine available data on related bacterial enzymes in terms of proposed reaction mechanisms and protein architecture. As summarized in the introduction, two main pathways of CLA production by bacteria are currently known - direct LA isomerization and a hydration-dehydration sequence. In the former case three enzymes - BFI, PFI and PAI were studied in details and major parallels in the reaction mechanisms were discussed in the previous chapter. Identities of the enzymes involved in LA hydration and further CLA formation are still not clear, and a simultaneous production of both hydroxy FA and CLA was observed for some microbial strains (Ogawa et al. 2005). A putative PUFA double bond isomerase gene was isolated from Lactobacillus acidophilus (LAI) and sequence was deposited to public databases (Cao 2005), the partial purification of this enzyme was also reported (Irmak et al. 2006). A set of highly similar gene sequences resulted from the BLAST search against this query (http://www.ncbi.nlm.nih.gov/BLAST/), most of them are annotated as members of 67-kDa myosin cross-reactive antigene (MCRA) family. Originally, the MCRA protein was demonstrated to be involved in the acute autoimmune response resulting from severe infections with S. pyogenes (Kil et al. 1994). It was localized to the outer bacterial membrane and was predicted to have a transmembrane helix, but the functional role apart from antigenic properties was not assigned. Given the high degree of similarity between MRCA and known PUFA double bond isomerases, it was of interest to test whether recombinant MRCA protein is active as FA-modifying enzyme. Sequence comparison between PAI, PFI and putative PUFA double bond isomerases from L. reuteri (LRI), B. breve (BBI) and S. pyogenes (MRCA, renamed as SPH) revealed relatively low similarity (below 30\%) outside of nucleotide-binding domain (Fig. 27), which may indicate conserved reaction mechanism but different protein structures for these enzymes. Three latter proteins are highly similar (more than $70 \%$ sequence identity) and therefore can be classified as a distinct group of PUFA double bond isomerases (group 2), opposed to PAI and PFI. 
Purified recombinant SPH protein contained a flavin-like cofactor (judged by UVVIS spectrum) and converted free PUFAs to 10-hydroxy-FAs (Figs. 30,31), which are putative precursors of CLA in vivo as described by several groups (Devillard et al. 2007, Kishimoto et al. 2003, Ogawa et al. 2001). The substrate preferences of SPH are strikingly similar to those of PAI, e.g. free carboxyl group and (9Z)-double bond must be present; but in contrast to PAI, PUFAs longer than C18 were not transformed by SPH. SPH essentially adds one water molecule to a (9Z)-double bond of the substrate, as demonstrated in experiments with isotopically labeled water (Fig. 29), the (12Z)-double bond can be hydrated to lesser extent, yielding dihydroxy-FA. This activity clearly depends on the presence of FAD, which likely binds to SPH via a divalent cation and therefore can be separated from the protein by repeated ultrafiltration cycles. Neither FMN nor 5-deaza-5-carba-FAD substituted FAD in the hydration reaction.

The hydratase activity towards (9Z)-double bond of FAs was described for several Pseudomonas sp. (Niehaus et al. 1970, Schroepfer et al. 1970) and 10-HO along with 10HOE were shown to be the products derived from OA and LA, respectively. SPH is the first protein demonstrated to be responsible for this activity, and thus it can be classified as a new member of carbon-oxygen lyases, which acts on non-activated carbon-carbon double bonds in contrast to well-studied enoyl-CoA hydratases, involved in FA $\beta$ oxidation. Stereospecificity of hydration and the identity of additional products found in the GC profiles of SPH reaction (Fig. 32) still remain to be determined.

Although unexpected, the hydratase activity of SPH shed light on the properties of a putative $(9 Z, 11 E)$-CLA producing isomerase BBI. Bifidobacterium and Lactobacillus species were demonstrated to produce exclusively $(9 Z, 11 E)$-CLA, but depending on growth conditions high amounts of 10-HOE may accumulate during LA biotransformation (Ogawa et al. 2005, Coakley et al. 2003). Recombinant BBI protein was purified as soluble His-tag fusion and again, the appearance of UV-VIS spectrum suggested that FAD is indeed present in the enzyme, as predicted by the sequence conservation of nucleotide binding domain. Despite this, BBI did not produce CLA when supplemented with LA and other PUFAs. Re-examination of GC-MS data and comparison with SPH reaction products revealed that low amounts of 10-HOE were formed by BBI when LA was the substrate (Supplem. Table 6). Tightly bound FAs, co- 
purifying with BBI, apparently account for this impaired activity. PA, SA and OA, found in BBI preparations (Fig. 33), also were shown to inhibit BFI isomerase activity by competitive inhibition mechanism (Kepler et al. 1970).

Combined data on microbial PUFA metabolism, enzymatic reactions and structure of PUFA double bond isomerase create the general picture of biosynthetic routes leading from PUFAs to conjugated PUFAs, including CLA. All CLA-producing enzymes fall in two classes - single proteins catalyzing the direct isomerization of C18-C20 PUFAs to conjugated products (PAI, PFI) and enzymatic systems involving hydration-dehydration steps, catalyzed either by a single polypeptide chain or a protein complex. They will be referred as group 1 and group 2 enzymes, respectively. The results presented here explain the function of group 1 enzymes as FAD-dependent isomerases, which transiently oxidize PUFA substrate likely forming flavin and PUFA radicals, with subsequent double bond isomerization and hydrogen re-addition from FAD to the conjugated PUFA radical. Since the studies on PFI enzyme were performed with highly pure protein displaying a flavinlike spectrum (Zheng et al. 2002), it is logical to assume that the PFI mechanism in general resembles that of PAI, the differences in stereochemistry and hydrogen exchange with solvent are probably due to the non-identical active site geometries, in agreement with low sequence conservation. Similar protein sequences and formation of hydroxy FA bring BBI, SPH and LRI along with other described Lactobacilli enzymes into group 2 of PUFA double bond isomerases. FAD binding and formation of hydroxy FA by SPH and BBI proteins was confirmed in the present work. It is likely that other group members show similar activity due the high sequence conservation between group 2 enzymes. Genomes of several pathogenic bacteria of Streptococcus, Staphylococcus and Propionibacterium species have ORFs annotated as MRCA-related proteins, which are highly similar to group 2 of PUFA double bond isomerases. Taking into account membrane localization demonstrated for SPH in S. pyogenes strains (Kil et al. 1994), these proteins might play not only structural role, but also participate in host-pathogen interaction, hence representing potential drug targets.

Assuming that group 2 enzymes indeed produce CLA by hydration-dehydration sequence, the principal question unanswered here is whether a hydratase-isomerase activity is encoded in a single polypeptide chain or additional protein partners are 
required for the formation of CLA. It is possible that recombinant SPH and BBI proteins are compromised due to the sub-optimal expression conditions in the heterologous host and/or missing cofactors, absent in E.coli. Equally plausible explanation is the involvement of a separate dehydratase protein, converting 10-HOE to CLA, which has no orthologs in E. coli. These options can be addressed by protein isolation from the native host, for example as was described by Kepler et al. in case of BFI, followed by identification of auxiliary proteins using mass spectrometry.

In summary, the structure of PAI presented here, not only revealed the mechanism of PUFA double bond isomerization in atomic details, but in combination with literature data also allowed to systematize the biosynthetic pathways of CLA formation into two distinct classes. Although much less in known about group 2 PUFA double bond isomerases, the framework experiments concerning PAI structure and reaction mechanism will be relevant for understanding these enzymes. 


\section{Summary and conclusions}

Extensive structural characterization of PAI, presented here, uncovered the details of chemically demanding double bond isomerization reaction in PUFAs. The inert aliphatic system is activated during catalysis via transient oxidation performed by FAD cofactor, which is non-covalently bound to PAI protein in equimolar stoichiometry. PAI requires a free carboxylic group in the PUFA substrate for its activity, which was also reported to be the case for PFI and BFI enzymes. In the structure of PAI-(10E,12Z)-CLA complex, the free FA carboxylate is recognized by two residues, Arg88 and Phe193, which act as a flexible substrate-driven "gate" at the entrance to the active site. Mutation of either of these residues to Ser and Ala, respectively, strongly affected catalytic efficiency of PAI, illustrating the importance of ionic interactions between Arg88 and free carboxylate of LA. The mode of $(10 E, 12 Z)$-CLA binding in the complex implies that pentadienyl moiety of LA is sandwiched between aromatic rings of Phe168 and FAD. Such substrate positioning brings the atoms $\mathrm{C} 11$ in LA and N5 in FAD within the distance of $3.2 \AA$, enabling the transfer of pro $R$-hydrogen from $\mathrm{C} 11$ to FAD as the initial step of isomerization reaction. An intermediate FA radical then undergoes allylic rearrangement and hydrogen is added back from FAD to the position C9. The direction of allylic shift is governed by Phe168, due to the closer distance between the latter and C9 rather than C13 carbon of FA, and therefore the mesomer with a partial positive charge on C9 is stabilized. Importantly, the regiospecificity of hydrogen abstraction relies on the distance between the Arg88-Phe93 lock and the N5 atom of FAD, explaining the preference of PAI towards the substrates with a $(9 Z, 12 Z)$-double bond system. Independent experiments with isotopically labeled LA confirmed that pro $R$-hydrogen is transferred from $\mathrm{C} 11$ to $\mathrm{C} 9$ position of LA during $(10 E, 12 Z)$-CLA formation. Generation of FAD radical in the active site of PAI was observed during anaerobic reduction of the enzyme, in addition the replacement of FAD by 5-deaza-5-carba-FAD abolished PAI activity. These data strongly suggest that a radical mechanism may be employed by PAI, rather than hydride transfer.

Based on sequence analysis and available biochemical data, PUFA double bond isomerases can be divided in two groups. The enzymes of group 1, including PAI and PFI, act by direct hydrogen transfer that depends on FAD, the role of the latter remains to 
be confirmed for PFI reaction. Group 2 includes a number of highly similar putative PUFA double bond isomerases related to MCRA. Among them, SPH, BBI and LRI proteins were characterized in this work in order to test whether mechanistic principles of PAI reaction hold true for all PUFA double bond isomerases. Recombinant SPH protein showed an unexpected double bond hydratase activity with the preferred substrates being C16-C18 FAs harboring a (9Z)-double bond and a free carboxylic group. SPH hydratase activity was FAD-dependent, confirming the identity of cofactor, but whether it plays only a structural role or is involved in the catalysis remains unclear. A flavin cofactor was also found in purified BBI protein, but despite apparent protein integrity, the catalytic efficiency was very low and only traces of mono-hydroxy FAs were detected in the reaction products. This was most likely due to the inhibitory effect of tightly associated FAs, co-purifying with BBI protein. Possible links between hydroxy FAs and CLA production were described in the literature at the level of microbial metabolism of exogenous LA, but no enzyme converting 10-HOE to CLA was published to date. Thus, further investigations on the mutual relationship of these two FA species during LA turnover by SPH and BBI might provide valuable information about PUFA metabolism by group 2 PUFA double bond isomerases from anaerobic bacteria. 


\section{Supplementary material.}

Supplementary Table 1

Oligos used for BBI, LRI, SPH, PFI and PAI cloning. Restriction sites are underlined.

\begin{tabular}{|c|c|c|c|c|c|}
\hline \multirow[t]{2}{*}{ ORF } & \multicolumn{2}{|r|}{ Oligos } & \multirow{2}{*}{$\begin{array}{l}\begin{array}{r}\text { Restriction } \\
\text { enzymes }\end{array} \\
\text { Nde I } \\
\text { Xho I }\end{array}$} & \multirow{2}{*}{\begin{tabular}{|c|} 
Vector \\
pET28a
\end{tabular}} & \multirow{2}{*}{\begin{tabular}{|l|} 
Resulting construct \\
pET28-BBI \\
N-terminal 6xHis tag
\end{tabular}} \\
\hline & $\begin{array}{l}\text { BBIfw_2 } \\
\text { BBIrv_1 }\end{array}$ & $\begin{array}{l}\text { 5'- aaacatatgtactacagcagcggcaactatgag-3' } \\
\text { 5'- tttctcgagttagatcacatggtattcgcgtagcagggtagc }-3 \text { ', }\end{array}$ & & & \\
\hline & $\begin{array}{l}\text { BBIfw_2 } \\
\text { BBIrv_2 }\end{array}$ & $\begin{array}{l}\text { 5'- aaacatatgtactacagcagcggcaactatgag-3' } \\
\text { 5'- aaactcgaggatcacatggtattcgcgtagcagg }-3^{\prime}\end{array}$ & $\begin{array}{l}\text { Nde I } \\
\text { Xho I }\end{array}$ & pET24a & $\begin{array}{l}\text { pET24-BBI } \\
\text { C-terminal 6xHis tag }\end{array}$ \\
\hline & $\begin{array}{l}\text { BBIfw_2 } \\
\text { BBIrv_1 }\end{array}$ & $\begin{array}{l}\text { 5'- aaacatatgtactacagcagcggcaactatgag-3' } \\
\text { 5'- tttctcgagttagatcacatggtattcgcgtagcagggtagc }-3 \text { ' }\end{array}$ & $\begin{array}{l}\text { Nde I } \\
\text { Xho I }\end{array}$ & pET24a & $\begin{array}{l}\text { pET24-BBI } \\
\text { no affinity tag }\end{array}$ \\
\hline & $\begin{array}{l}\text { LRIfw_1 } \\
\text { LRIrv_1 }\end{array}$ & $\begin{array}{l}5^{\prime} \text { - aaagaattcatgtattattcaaacgggaattatg }-3^{\prime} \\
5^{\prime}-\text { tttgcggccgcttatagtaagtgctgttgctccattaattcaccaatcc }-3^{\prime}\end{array}$ & $\begin{array}{l}\text { EcoR I } \\
\text { Not I }\end{array}$ & pGEX6-1 & $\begin{array}{l}\text { pGEX6-LRI } \\
\text { N-terminal GST tag }\end{array}$ \\
\hline LRI & $\begin{array}{l}\text { LRIfw_2 } \\
\text { LRIrv_1 }\end{array}$ & $\begin{array}{l}\text { 5'- aaacatatgtattattcaaacgggaattatgaagc }-3^{\prime} \\
5 \text { '- tttgcggccgcttatagtaagtgctgttgctccattaattcaccaatcc -3' }\end{array}$ & $\begin{array}{l}\text { Nde I } \\
\text { NotI }\end{array}$ & pET28a & $\begin{array}{l}\text { pET28-LRI } \\
\text { N-terminal 6xHis tag }\end{array}$ \\
\hline
\end{tabular}




\begin{tabular}{|c|c|c|c|c|c|}
\hline & $\begin{array}{l}\text { LRIfw_2 } \\
\text { LRIrv_2 }\end{array}$ & $\begin{array}{l}\text { 5'- aaacatatgtattattcaaacgggaattatgaagc }-3^{\prime} \\
5 \text { '- tttgcggccgctagtaagtgctgttgctccattaattcaccaatcc }-3 \text { ' }\end{array}$ & $\begin{array}{l}\text { Nde I } \\
\text { NotI }\end{array}$ & pET24a & $\begin{array}{l}\text { pET24-LRI } \\
\text { C-terminal 6xHis tag }\end{array}$ \\
\hline & $\begin{array}{l}\text { LRIfw_2 } \\
\text { LRIrv_1 }\end{array}$ & $\begin{array}{l}\text { 5'- aaacatatgtattattcaaacgggaattatgaagc }-3{ }^{\prime} \\
5^{\prime}-\text { tttgcggccgcttatagtaagtgctgttgctccattaattcaccaatcc }-3 \text {, }\end{array}$ & $\begin{array}{l}\text { Nde I } \\
\text { NotI }\end{array}$ & pET24a & $\begin{array}{l}\text { pET24-LRI } \\
\text { no affinity tag }\end{array}$ \\
\hline & $\begin{array}{l}\text { SPHfw_1 } \\
\text { SPHrv_1 }\end{array}$ & $\begin{array}{l}\text { 5'-aaagctagcatgtattatactagtggtaattacg-3' } \\
\text { 5'- aaagcggccgcttacataagattagcatctttgagc }\end{array}$ & $\begin{array}{l}\text { Nhe I } \\
\text { Not I }\end{array}$ & pET28a & $\begin{array}{l}\text { pET28-SPH } \\
\text { N-terminal 6xHis tag }\end{array}$ \\
\hline SPH & $\begin{array}{l}\text { SPHfw_1 } \\
\text { SPHrv_2 }\end{array}$ & $\begin{array}{l}\text { 5'-aaagctagcatgtattatactagtggtaattacg-3' } \\
\text { 5'- aaagcggccgccataagattagcatctttgagc }-3 \text { ' }\end{array}$ & $\begin{array}{l}\text { Nhe I } \\
\text { Not I }\end{array}$ & pET24a & $\begin{array}{l}\text { pET24-SPH } \\
\text { C-terminal 6xHis tag }\end{array}$ \\
\hline & $\begin{array}{l}\text { SPHfw_1 } \\
\text { SPHrv_1 }\end{array}$ & $\begin{array}{l}\text { 5'-aaagctagcatgtattatactagtggtaattacg-3' } \\
\text { 5'- aaagcggccgcttacataagattagcatctttgagc-3' }\end{array}$ & $\begin{array}{l}\text { Nhe I } \\
\text { Not I }\end{array}$ & pET24a & $\begin{array}{l}\text { pET24-SPH } \\
\text { no affinity tag }\end{array}$ \\
\hline & $\begin{array}{l}\text { PAI full } \\
\text { length }\end{array}$ & $\begin{array}{l}\text { 5'-aagcggccgcatgtccatctcgaaggattcacgtatc-3' } \\
\text { 5'-aactcgagtcacacgaagaaccgcgtcaccaggtcc-3' }\end{array}$ & $\begin{array}{l}\text { Not I } \\
\text { Xho I }\end{array}$ & pET24a & $\begin{array}{l}\text { pET24-CsLOX-PAI } \\
\text { pET24-OcLOX-PAI }\end{array}$ \\
\hline $\begin{array}{l}\text { PAI } \\
\text { fusions }\end{array}$ & $\begin{array}{l}\text { Cs_Lox } \\
\text { N-Term }\end{array}$ & $\begin{array}{l}\text { 5'-aatgatcaatgtttggaattgggaagaacatcattgaag-3' } \\
\text { 5'-aagcggccgcttgtttgacttggaagataaacatgattg-3' }\end{array}$ & Bcl I & pET24a & \\
\hline $\begin{array}{l}\text { with } \\
\text { LOX }\end{array}$ & $\begin{array}{l}\text { Oc_Lox } \\
\text { N-Term }\end{array}$ & $\begin{array}{l}\text { 5'-aatgatcaatgggtgtctatcgegtctgcgtgtccacc-3' } \\
5 \text { '-aagcggccgctccegaccgtggtgcagccaatgcccac-3' }\end{array}$ & Bcl I & pET24a & \\
\hline
\end{tabular}




\begin{tabular}{|c|c|c|c|c|c|}
\hline domains & $\begin{array}{l}\text { PFIfw_1 } \\
\text { PFIrv_1 }\end{array}$ & $\begin{array}{l}\text { 5'-aaaggatccatgcatgattttgatgacacgattgc-3' } \\
\text { 5'-aaagcggccgcttagaaaaaatccttgatcaaattatc-3' }\end{array}$ & $\begin{array}{l}\text { BamHI } \\
\text { NotI }\end{array}$ & pGEX6-1 & $\begin{array}{l}\text { pGEX6-PFI } \\
\text { N-terminal GST tag }\end{array}$ \\
\hline PFI & $\begin{array}{l}\text { PFIfw_2 } \\
\text { PFIrv_1 }\end{array}$ & $\begin{array}{l}\text { 5'-aaacatatgtctttgaatagagttcttcacattttcc-3' } \\
\text { 5'-aaagcggccgcttagaaaaaatccttgatcaaattatc-3' }\end{array}$ & $\begin{array}{l}\text { NdeI } \\
\text { NotI }\end{array}$ & pET28a & $\begin{array}{l}\text { pET24a-PFI } \\
\text { N-terminal His tag }\end{array}$ \\
\hline & $\begin{array}{l}\text { PFIfw_2 } \\
\text { PFIrv_1 }\end{array}$ & $\begin{array}{l}\text { 5'-aaacatatgtctttgaatagagttcttcacattttcc-3' } \\
\text { 5'-aaagcggccgcttagaaaaaatccttgatcaaattatc-3' }\end{array}$ & $\begin{array}{l}\text { NdeI } \\
\text { NotI }\end{array}$ & pET24a & $\begin{array}{l}\text { pET24a-PFI } \\
\text { no affinity tag }\end{array}$ \\
\hline
\end{tabular}


Supplementary Table 2

Oligos used for PAI site-directed mutagenesis and fused constructs with LOX domains. Restriction sites are underlined where silent mutation was introduced.

\begin{tabular}{|c|c|c|}
\hline Mutation & Oligos & Restriction enzyme \\
\hline Arg88-Ala & $\begin{array}{l}\text { 5'-cgacgggccgaaactgcgtagcgagttcctgcacgagg-3' } \\
\text { 5'-cctcgtgcaggaactcgctacgcagtttcggcccgtcg-3' }\end{array}$ & Nru I \\
\hline Arg88-Ser & $\begin{array}{l}\text { 5'-cgacgggccgaaactgcgtagcgagttcetgcacgagg-3' } \\
5^{\prime} \text {-cctcgtgcaggaactcgctacgcagtttcggcccgtcg-3' }\end{array}$ & Nru I \\
\hline Phe193-Ala & $\begin{array}{l}\text { 5'-cttcgtcaccatgatgtccgcggccaagggagacctg-3' } \\
\text { 5'-caggtctcccttggccgcggacatcatggtgacgaag-3' }\end{array}$ & Bgl II \\
\hline he168-Leu & $\begin{array}{l}\text { 5'-ggatcaaccccttcacagcgctcggctacgggcacttcgac-3' } \\
\text { 5'-gtcgaagtgcccgtagccgagcgctgtgaaggggttgatcc-3' }\end{array}$ & Eco47 III \\
\hline $\begin{array}{l}\text { Phe168Gly169- } \\
\text { Gly168Phe169 }\end{array}$ & $\begin{array}{l}5 \text { '-tggatcaacccttcactgcaggcttctacgggcacttcgacaacg-3' } \\
5 \text { '-cgttgtcgaagtgcccgtagaagcttgcagtgaaggggttgatcca-3' }\end{array}$ & Pst I \\
\hline
\end{tabular}


Supplementary Table 3.

Fractional coordinates of the ten most occupied iodide sites as determined by SHELXD.

\begin{tabular}{|l|l|l|l|l|}
\hline Site & $\mathrm{x}$ & $\mathrm{y}$ & $\mathrm{z}$ & Occupancy \\
\hline 1 & 0.232 & 0.101 & 0.250 & 1.00 \\
\hline 2 & 0.250 & 0.112 & 0.500 & 0.47 \\
\hline 3 & 0.356 & 0.153 & 0.448 & 0.93 \\
\hline 4 & 0.167 & 0.022 & 0.128 & 0.67 \\
\hline 5 & 0.163 & 0.074 & 0.050 & 0.62 \\
\hline 6 & 0.370 & 0.209 & 0.456 & 0.55 \\
\hline 7 & 0.124 & 0.039 & -0.046 & 0.41 \\
\hline 8 & 0.213 & 0.006 & 0.220 & 0.41 \\
\hline 9 & 0.381 & 0.067 & 0.478 & 0.40 \\
\hline 10 & 0.158 & 0.021 & 0.211 & 0.37 \\
\hline
\end{tabular}


Supplementary Table 4.

Reaction products of PAI wt and selected mutants with unpolar lipids.

\begin{tabular}{|c|c|c|}
\hline Protein & Substrate & Product \\
\hline $\begin{array}{l}\text { PAI wt } \\
\text { R88S, F193A, R88S+F193A }\end{array}$ & Methyl ester LA & $\begin{array}{l}(10 E, 12 Z)-C L A(\text { traces }) \\
(10 E, 12 Z)-C L A(3-5 \%)\end{array}$ \\
\hline $\begin{array}{l}\text { PAI wt } \\
\text { R88S, F193A, R88S+F193A }\end{array}$ & Linoleyl-CoA & $\begin{array}{l}(10 \mathrm{E}, 12 \mathrm{Z})-\mathrm{CLA}-\mathrm{CoA}(30 \%) \\
(10 \mathrm{E}, 12 \mathrm{Z})-\mathrm{CLA}-\mathrm{CoA}(10-30 \%)\end{array}$ \\
\hline $\begin{array}{l}\text { PAI wt } \\
\text { R88S, F193A, R88S+F193A }\end{array}$ & $\begin{array}{l}\text { Tri-linoleyl- } \\
\text { glycerol }\end{array}$ & $\begin{array}{l}\text { None } \\
\text { None }\end{array}$ \\
\hline $\begin{array}{l}\text { PAI wt } \\
\text { R88S, F193A, R88S+F193A }\end{array}$ & $\begin{array}{l}\text { Di-linoleyl- } \\
\text { phosphatidylcholine }\end{array}$ & $\begin{array}{l}\text { None } \\
\text { None }\end{array}$ \\
\hline PAI wt & Linoleyl alcohol & Conjugated product \\
\hline
\end{tabular}


Supplementary Table 5. Reaction products of SPH purified as 6x His tag fusion protein.

\begin{tabular}{|c|c|c|c|}
\hline Substrate & Products & $\mathrm{RT}, \min$ & MS fragments, $\mathrm{m} / \mathrm{z}$ \\
\hline $16: 1^{\Delta 9 Z}(\mathrm{PA})$ & $10-\mathrm{HH}$ & 8.13 & $357(\mathrm{M}-1)^{+}, 273,187,169$ \\
\hline $18: 1^{\Delta 9 Z}(\mathrm{OA})$ & $10-\mathrm{HO}$ & 9.56 & $385(\mathrm{M}-1)^{+}, 273,215,169$ \\
\hline $18: 1^{\Delta 9 E}$ & No products & - & - \\
\hline $18: 1^{\Delta 11 Z}$ & No products & - & - \\
\hline $18: 2^{\Delta 9 Z, \Delta 12 Z}(\mathrm{LA})$ & $\begin{array}{l}\text { 10-HOE } \\
10,13-\text { di-HO }\end{array}$ & $\begin{array}{l}9.84 \\
10.41\end{array}$ & $\begin{array}{l}383(\mathrm{M}-1)^{+}, 273,213,173, \\
169,313,273,213,173\end{array}$ \\
\hline $18: 2^{\Delta 9 E, \Delta 12 E}$ & No products & - & - \\
\hline $18: 2^{\Delta 9 Z, \Delta I I E}$ & No products & - & - \\
\hline $18: 2^{\Delta 10 E, \Delta 12 Z}$ & No products & - & - \\
\hline $\begin{array}{l}18: 2^{\Delta 9 Z, \Delta 12 Z} \\
\text { methyl ester }\end{array}$ & No products & - & - \\
\hline $18: 3^{\Delta 9 Z, \Delta 12 Z, \Delta 15 Z}$ & $\begin{array}{l}\text { (12Z,15Z)-10-hydroxy- } \\
\text { octadecadienoate }\end{array}$ & 10.35 & $380(\mathrm{M}-1)^{+}, 273,241,169$ \\
\hline $18: 3^{\Delta 6 Z, \Delta 9 Z, \Delta 12 Z}$ & No products & - & - \\
\hline $20: 3^{\Delta 11 Z, \Delta 14 Z, \Delta 17 Z}$ & No products & - & - \\
\hline $20: 4^{\Delta 5 Z, \Delta 8 Z, \Delta 11 Z, \Delta 14 Z}$ & No products & - & - \\
\hline $22: 5^{\Delta 5 Z, \Delta 8 Z, \Delta 11 Z, \Delta 14 Z, \Delta 17 Z}$ & No products & - & - \\
\hline $\begin{array}{l}\text { Di-linoleyl- } \\
\text { phosphatidylcholine }\end{array}$ & No products & - & - \\
\hline Tri-linoleyl-glycerol & No products & - & - \\
\hline
\end{tabular}


Supplementary Table 6. Reaction products of BBI purified as 6x His tag fusion protein.

\begin{tabular}{|l|l|l|l|}
\hline Substrate & Product & RT, min & MS fragments, m/z \\
\hline $14: 1^{\Delta 9 Z}$ & No product & - & - \\
\hline $16: 1^{\Delta 9 Z}(\mathrm{PA})$ & $10-\mathrm{HH}$ & 8.13 & $357(\mathrm{M}-1)^{+}, 273,187,169$ \\
\hline $18: 1^{\Delta 9 Z}(\mathrm{OA})$ & $10-\mathrm{HO}$ & 9.56 & $385(\mathrm{M}-1)^{+}, 273,215,169$ \\
\hline $18: 1^{\Delta 11 Z}$ & No products & - & - \\
\hline $18: 1^{\Delta 9 E}$ & No products & - & - \\
\hline $18: 2^{\Delta 9 Z, 12 Z}(\mathrm{LA})$ & $10-\mathrm{HOE}$ & 9.84 & $383(\mathrm{M}-1)^{+}, 273,213,173,169$ \\
\hline $18: 2^{\Delta 9 Z, \Delta 12 Z}$ & No products & - & - \\
\hline methyl ester & & & \\
\hline CLA & No products & - & - \\
\hline $18: 2^{\Delta 9 E, I 2 E}$ & No products & - & - \\
\hline $18: 3^{\Delta 9 Z, 12 Z, 15 Z}$ (LeA) & No products & - & - \\
\hline $20: 4^{\Delta 5 Z, 8 Z, 11 Z, 14 Z}(\mathrm{AA})$ & No products & - & - \\
\hline
\end{tabular}


SPH : MYYT SGNYEAFATPRKPEGVDCKSAYIVGTGLAGLAAAVFLIRDGHMAGERIHLFEELPLAGGSLDGIEKPHLGFUTRGGREMENHFECMTMDMY : 94
100
120
140
160
180

SPH : RSIP SLEIPGASYLDEFYNLDKDDPNGSNCRLIHE RGNRVDDDGCYTLGKC KELVHLDMKTEESLGDCTIEEFFSEDFFKSNFTUYMATMFAF : 188

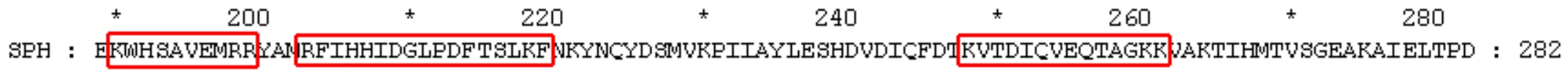
* 300
$+\quad 320$
340
360

SPH : DLVFUTNGSITESSTYGSHHEVAKPTKALGGSTNLTENLAACSDDFGHPKVFYCDLPAESTFVSATATIRHPAIEPYIERLTHRDLHDGKVNTG : 376
380
400
420
440

460

SEH : GITITDSNMMMSFAIHRCPHFKECKDNETTVNIYGLYSNSEGNYVHKKIEECTGCEITEEWLYHLGVPVDKIKDLASCDYINTVPVMMPYITS : 470
480
500
520
540
560

SPH : YFMPRUKGDRPKUIPDGSUNLAFIGNFAESERRDTVFTTEYSIRTAMEAVYSFLNVERGFPEVFNGAYDIRELIRAFYYLNDKKAIKDMDLPIA : 564

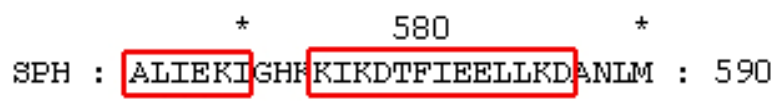

Supplementary Fig. 1. Analysis of SPH protein integrity by mass spectrometry.

Fragments identified by MALDI-TOF mass spectrometry are labeled with red boxes in SPH aminoacid sequence.

C-terminal part is retained in the purified protein. 

100
120
140
160
180

BBI : RSIP SIETEGVSVLDEYYWLN KEDFNYSLCRARKDLGKDAGI KGKFGLSDKASMEDMKIFFTPDEDLYDKPITDFFDDEVLNSNFTULYURTMFA : 188

* $200 \quad * \quad 220 \quad * \quad 240 \quad$ * $260 \quad$ *

BBI : FENWHGALEMKLYTKRYIHHIGGLPDF SALRFTRYNCYESMILPMT KYLESHGVEFRYNTKVENVEFAIGGGDGERREHTGVGCDTICKICATS : 282

$\begin{array}{ccccccc}* & 300 & * & 320 & * & 340 & *\end{array}$

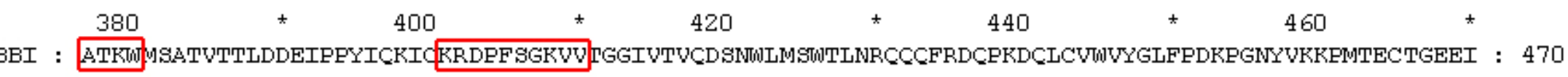
500
520
540
560

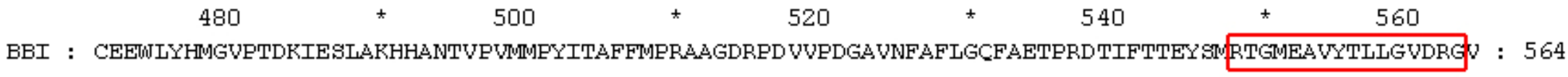

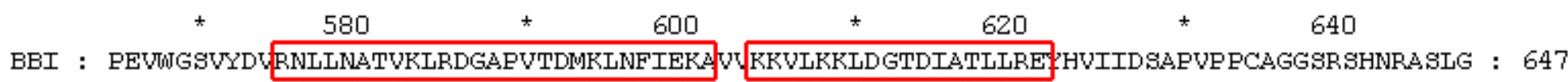

Supplementary Fig. 2. Analysis of SPH protein integrity by mass spectrometry.

Fragments identified by MALDI-TOF mass spectrometry are labeled with red boxes in BBI aminoacid sequence.

C-terminal part is retained in the purified protein. 


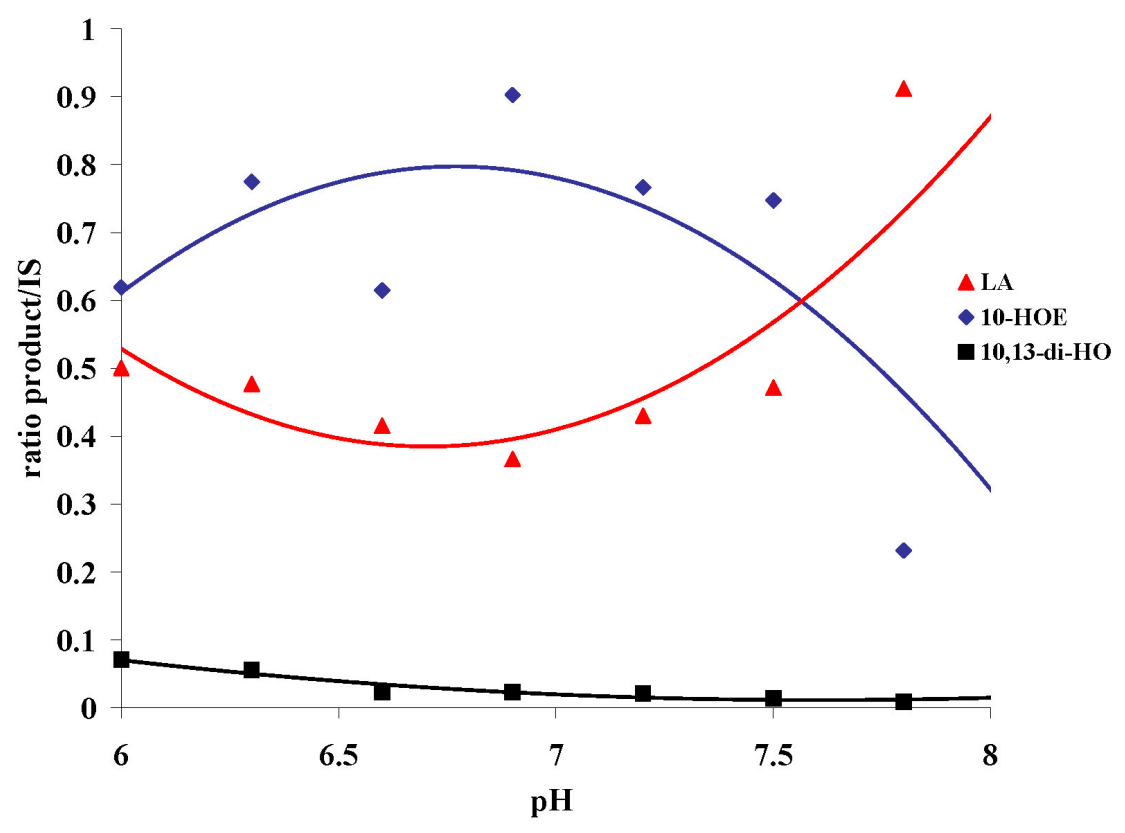

Supplementary Fig. 3. pH dependence of LA hydration by SPH.

Reaction was performed in duplicates, FA extracted and analyzed by GC-MS. The ratio of LA and each product to internal standard (AA) is plotted as function of $\mathrm{pH}$. Red - LA, blue - 10-HOE, black 10,13-di-HO. 


\section{References}

(1) Samuelsson, B. (1987) An elucidation of the arachidonic acid cascade. Discovery of prostaglandins, thromboxane and leukotrienes. Drugs 33 Suppl 1, 2-9.

(2) Turner, J. G., Ellis, C., and Devoto, A. (2002) The jasmonate signal pathway. Plant Cell 14 Suppl, S153-164.

(3) Ha, Y. L., Grimm, N. K., and Pariza, M. W. (1987) Anticarcinogens from fried ground beef: heat-altered derivatives of linoleic acid. Carcinogenesis 8, 1881-1887.

(4) Yurawecz, M. P., Mossoba, M.M., Kramer, J.K.G., Pariza, M.W., Nelson, G.J., eds. (1999) Advances in conjugated linoleic acid research, Vol. 1, Champaign : AOCS Press.

(5) Pariza, M. W. (2004) Perspective on the safety and effectiveness of conjugated linoleic acid. Am J Clin Nutr 79, 1132S-1136S.

(6) Wahle, K. W., Heys, S. D., and Rotondo, D. (2004) Conjugated linoleic acids: are they beneficial or detrimental to health? Prog Lipid Res 43, 553-587.

(7) Pariza, M. W., Park, Y., and Cook, M. E. (2000) Mechanisms of action of conjugated linoleic acid: evidence and speculation. Proc Soc Exp Biol Med 223, 8-13.

(8) Pariza, M. W., Y. Park, X. Xu, J. Ntambi, and K. Kang. (2003) Advances in Conjugated Linoleic Acid Research, Vol. 2, AOCS Press, Champaign

(9) Park, Y., Albright, K. J., Liu, W., Storkson, J. M., Cook, M. E., and Pariza, M. W. (1997) Effect of conjugated linoleic acid on body composition in mice. Lipids 32, 853-858.

(10) Park, Y., Storkson, J. M., Albright, K. J., Liu, W., and Pariza, M. W. (1999) Evidence that the trans-10,cis-12 isomer of conjugated linoleic acid induces body composition changes in mice. Lipids 34, 235-241.

(11) Pariza, M. W., Park, Y., and Cook, M. E. (2001) The biologically active isomers of conjugated linoleic acid. Prog Lipid Res 40, 283-298.

(12) Park, Y., Storkson, J. M., Liu, W., Albright, K. J., Cook, M. E., and Pariza, M. W. (2004) Structure-activity relationship of conjugated linoleic acid and its cognates in inhibiting heparin-releasable lipoprotein lipase and glycerol release from fully differentiated 3T3-L1 adipocytes. $J$ Nutr Biochem 15, 561-568.

(13) Evans, M., Lin, X., Odle, J., and McIntosh, M. (2002) Trans-10, cis-12 conjugated linoleic acid increases fatty acid oxidation in 3T3-L1 preadipocytes. $J$ Nutr 132, 450-455.

(14) Granlund, L., Pedersen, J. I., and Nebb, H. I. (2005) Impaired lipid accumulation by trans10, cis12 CLA during adipocyte differentiation is dependent on timing and length of treatment. Biochim Biophys Acta 1687, 11-22.

(15) Whigham, L. D., Watras, A. C., and Schoeller, D. A. (2007) Efficacy of conjugated linoleic acid for reducing fat mass: a meta-analysis in humans. Am J Clin Nutr 85, 1203-1211.

(16) Larsen, T. M., Toubro, S., and Astrup, A. (2003) Efficacy and safety of dietary supplements containing CLA for the treatment of obesity: evidence from animal and human studies. J Lipid Res 44, 2234-2241.

(17) Yang, M., and Cook, M. E. (2003) Dietary conjugated linoleic acid decreased cachexia, macrophage tumor necrosis factor-alpha production, and modifies splenocyte cytokines production. Exp Biol Med (Maywood) 228, 51-58. 
(18) Yu, Y., Correll, P. H., and Vanden Heuvel, J. P. (2002) Conjugated linoleic acid decreases production of pro-inflammatory products in macrophages: evidence for a PPAR gamma-dependent mechanism. Biochim Biophys Acta 1581, 89-99.

(19) Ringseis, R., Muller, A., Herter, C., Gahler, S., Steinhart, H., and Eder, K. (2006) CLA isomers inhibit TNFalpha-induced eicosanoid release from human vascular smooth muscle cells via a PPARgamma ligand-like action. Biochim Biophys Acta 1760, 290-300.

(20) Ma, D. W., Field, C. J., and Clandinin, M. T. (2002) An enriched mixture of trans-10,cis-12-CLA inhibits linoleic acid metabolism and PGE2 synthesis in MDAMB-231 cells. Nutr Cancer 44, 203-212.

(21) Shen, C. L., Dunn, D. M., Henry, J. H., Li, Y., and Watkins, B. A. (2004) Decreased production of inflammatory mediators in human osteoarthritic chondrocytes by conjugated linoleic acids. Lipids 39, 161-166.

(22) Dinarello, C. A. (1999) Cytokines as endogenous pyrogens. J Infect Dis 179 Suppl 2, S294-304.

(23) Banni, S., Petroni, A., Blasevich, M., Carta, G., Cordeddu, L., Murru, E., Melis, M. P., Mahon, A., and Belury, M. A. (2004) Conjugated linoleic acids (CLA) as precursors of a distinct family of PUFA. Lipids 39, 1143-1146.

(24) Park, Y., Storkson, J. M., Albright, K. J., Liu, W., and Pariza, M. W. (2005) Biological activities of conjugated fatty acids: conjugated eicosadienoic (conj. 20:2delta(c11,t13/t12,c14)), eicosatrienoic (conj. 20:3delta(c8,t12,c14)), and heneicosadienoic (conj. 21:2delta(c12,t14/c13,t15)) acids and other metabolites of conjugated linoleic acid. Biochim Biophys Acta 1687, 120-129.

(25) Akahoshi, A., Koba, K., Ichinose, F., Kaneko, M., Shimoda, A., Nonaka, K., Yamasaki, M., Iwata, T., Yamauchi, Y., Tsutsumi, K., and Sugano, M. (2004) Dietary protein modulates the effect of CLA on lipid metabolism in rats. Lipids 39, 25-30.

(26) Loscher, C. E., Draper, E., Leavy, O., Kelleher, D., Mills, K. H., and Roche, H. M. (2005) Conjugated linoleic acid suppresses NF-kappa B activation and IL-12 production in dendritic cells through ERK-mediated IL-10 induction. J Immunol 175, 4990-4998.

(27) Hwang, D. M., Kundu, J. K., Shin, J. W., Lee, J. C., Lee, H. J., and Surh, Y. J. (2007) cis-9,trans-11-conjugated linoleic acid down-regulates phorbol ester-induced NF-kappaB activation and subsequent COX-2 expression in hairless mouse skin by targeting IkappaB kinase and PI3K-Akt. Carcinogenesis 28, 363-371.

(28) Song, H. J., Sneddon, A. A., Heys, S. D., and Wahle, K. W. (2006) Induction of apoptosis and inhibition of NF-kappaB activation in human prostate cancer cells by the cis-9, trans-11 but not the trans-10, cis-12 isomer of conjugated linoleic acid. Prostate 66, 839-846.

(29) Wahle, K. W., and Heys, S. D. (2002) Cell signal mechanisms, conjugated linoleic acids (CLAs) and anti-tumorigenesis. Prostaglandins Leukot Essent Fatty Acids 67, 183-186.

(30) Kim, J. H., Hubbard, N. E., Ziboh, V., and Erickson, K. L. (2005) Conjugated linoleic acid reduction of murine mammary tumor cell growth through 5hydroxyeicosatetraenoic acid. Biochim Biophys Acta 1687, 103-109. 
(31) Javadi, M., Beynen, A. C., Hovenier, R., Lankhorst, A., Lemmens, A. G., Terpstra, A. H., and Geelen, M. J. (2004) Prolonged feeding of mice with conjugated linoleic acid increases hepatic fatty acid synthesis relative to oxidation. $J$ Nutr Biochem 15, 680-687.

(32) Purushotham, A., Shrode, G. E., Wendel, A. A., Liu, L. F., and Belury, M. A. (2007) Conjugated linoleic acid does not reduce body fat but decreases hepatic steatosis in adult Wistar rats. J Nutr Biochem.

(33) Taylor, C. G., and Zahradka, P. (2004) Dietary conjugated linoleic acid and insulin sensitivity and resistance in rodent models. Am J Clin Nutr 79, 1164S-1168S.

(34) Riserus, U. (2006) Trans fatty acids and insulin resistance. Atheroscler Suppl 7, 37-39.

(35) Kamphuis, M. M., Lejeune, M. P., Saris, W. H., and Westerterp-Plantenga, M. S. (2003) The effect of conjugated linoleic acid supplementation after weight loss on body weight regain, body composition, and resting metabolic rate in overweight subjects. Int J Obes Relat Metab Disord 27, 840-847.

(36) Kamphuis, M. M., Lejeune, M. P., Saris, W. H., and Westerterp-Plantenga, M. S. (2003) Effect of conjugated linoleic acid supplementation after weight loss on appetite and food intake in overweight subjects. Eur J Clin Nutr 57, 1268-1274.

(37) Riserus, U., Basu, S., Jovinge, S., Fredrikson, G. N., Arnlov, J., and Vessby, B. (2002) Supplementation with conjugated linoleic acid causes isomer-dependent oxidative stress and elevated C-reactive protein: a potential link to fatty acid-induced insulin resistance. Circulation 106, 1925-1929.

(38) Riserus, U., Vessby, B., Arnlov, J., and Basu, S. (2004) Effects of cis-9,trans-11 conjugated linoleic acid supplementation on insulin sensitivity, lipid peroxidation, and proinflammatory markers in obese men. Am J Clin Nutr 80, 279-283.

(39) Yurawecz, M. P., Kramer, J.K.G., Gudmundsen, O., Pariza, M.W., Banni, S., eds. (2006) Advances in Conjugated Linoleic Acid Research, Vol. 3.

(40) Devillard, E., McIntosh, F. M., Duncan, S. H., and Wallace, R. J. (2007) Metabolism of linoleic acid by human gut bacteria: different routes for biosynthesis of conjugated linoleic acid. J Bacteriol 189, 2566-2570.

(41) Wallace, R. J., McKain, N., Shingfield, K. J., and Devillard, E. (2007) Isomers of conjugated linoleic acids are synthesized via different mechanisms in ruminal digesta and bacteria. J Lipid Res.

(42) Griinari, J. M., Corl, B. A., Lacy, S. H., Chouinard, P. Y., Nurmela, K. V., and Bauman, D. E. (2000) Conjugated linoleic acid is synthesized endogenously in lactating dairy cows by Delta(9)-desaturase. J Nutr 130, 2285-2291.

(43) Kuhnt, K., Kraft, J., Moeckel, P., and Jahreis, G. (2006) Trans-11-18: 1 is effectively Delta9-desaturated compared with trans-12-18: 1 in humans. Br J Nutr 95, 752-761.

(44) Reiser, R. (1951) Federation Proc. 10, 236.

(45) Kepler, C. R., Hirons, K. P., McNeill, J. J., and Tove, S. B. (1966) Intermediates and products of the biohydrogenation of linoleic acid by Butyrinvibrio fibrisolvens. $J$ Biol Chem 241, 1350-1354.

(46) Kepler, C. R., and Tove, S. B. (1967) Biohydrogenation of unsaturated fatty acids. 3. Purification and properties of a linoleate delta-12-cis, delta-11-trans-isomerase from Butyrivibrio fibrisolvens. J Biol Chem 242, 5686-5692. 
(47) Kepler, C. R., Tucker, W. P., and Tove, S. B. (1970) Biohydrogenation of unsaturated fatty acids. IV. Substrate specificity and inhibition of linoleate delta-12cis, delta-11-trans-isomerase from Butyrivibrio fibrisolvens. J Biol Chem 245, 36123620 .

(48) Kepler, C. R., Tucker, W. P., and Tove, S. B. (1971) Biohydrogenation of unsaturated fatty acids. V. Stereospecificity of proton addition and mechanism of action of linoleic acid delta 12-cis, delta 11-trans-isomerase from Butyrivibrio fibrisolvens. J Biol Chem 246, 2765-2771.

(49) Hughes, P. E., Hunter, W. J., and Tove, S. B. (1982) Biohydrogenation of unsaturated fatty acids. Purification and properties of cis-9,trans-11-octadecadienoate reductase. J Biol Chem 257, 3643-3649.

(50) Fukuda, S., Suzuki, Y., Komori, T., Kawamura, K., Asanuma, N., and Hino, T. (2007) Purification and gene sequencing of conjugated linoleic acid reductase from a gastrointestinal bacterium, Butyrivibrio fibrisolvens. J Appl Microbiol 103, 365-371.

(51) Lopez, A., and Gerwick, W. H. (1987) Two new icosapentaenoic acids from the temperate red seaweed Ptilota filicina J. Agardh. Lipids 22, 190-194.

(52) Wise, M. L., Hamberg, M., and Gerwick, W. H. (1994) Biosynthesis of conjugated triene-containing fatty acids by a novel isomerase from the red marine alga Ptilota filicina. Biochemistry 33, 15223-15232.

(53) Wise, M. L., Rossi, J., and Gerwick, W. H. (1997) Characterization of the substrate binding site of polyenoic fatty acid isomerase, a novel enzyme from the marine alga Ptilota filicina. Biochemistry 36, 2985-2992.

(54) Zheng, W., Wise, M. L., Wyrick, A., Metz, J. G., Yuan, L., and Gerwick, W. H. (2002) Polyenoic fatty acid isomerase from the marine alga Ptilota filicina: protein characterization and functional expression of the cloned cDNA. Arch Biochem Biophys 401, 11-20.

(55) Kishimoto, N., Yamamoto, I., Toraishi, K., Yoshioka, S., Saito, K., Masuda, H., and Fujita, T. (2003) Two distinct pathways for the formation of hydroxy FA from linoleic acid by lactic acid bacteria. Lipids 38, 1269-1274.

(56) Ogawa, J., Matsumura, K., Kishino, S., Omura, Y., and Shimizu, S. (2001) Conjugated linoleic acid accumulation via 10-hydroxy-12-octadecaenoic acid during microaerobic transformation of linoleic acid by Lactobacillus acidophilus. Appl Environ Microbiol 67, 1246-1252.

(57) Ogawa, J., Kishino, S., Ando, A., Sugimoto, S., Mihara, K., and Shimizu, S. (2005) Production of conjugated fatty acids by lactic acid bacteria. J Biosci Bioeng $100,355-364$.

(58) Cahoon, E. B., Carlson, T. J., Ripp, K. G., Schweiger, B. J., Cook, G. A., Hall, S. E., and Kinney, A. J. (1999) Biosynthetic origin of conjugated double bonds: production of fatty acid components of high-value drying oils in transgenic soybean embryos. Proc Natl Acad Sci U S A 96, 12935-12940.

(59) Dyer, J. M., Chapital, D. C., Kuan, J. C., Mullen, R. T., Turner, C., McKeon, T. A., and Pepperman, A. B. (2002) Molecular analysis of a bifunctional fatty acid conjugase/desaturase from tung. Implications for the evolution of plant fatty acid diversity. Plant Physiol 130, 2027-2038. 
(60) Cahoon, E. B., Ripp, K. G., Hall, S. E., and Kinney, A. J. (2001) Formation of conjugated delta8, delta10-double bonds by delta12-oleic-acid desaturase-related enzymes: biosynthetic origin of calendic acid. J Biol Chem 276, 2637-2643.

(61) Fritsche, K., Hornung, E., Peitzsch, N., Renz, A., and Feussner, I. (1999) Isolation and characterization of a calendic acid producing $(8,11)$-linoleoyl desaturase. FEBS Lett 462, 249-253.

(62) Hornung, E., Pernstich, C., and Feussner, I. (2002) Formation of conjugated Delta11Delta13-double bonds by Delta12-linoleic acid (1,4)-acyl-lipid-desaturase in pomegranate seeds. Eur J Biochem 269, 4852-4859.

(63) Iwabuchi, M., Kohno-Murase, J., and Imamura, J. (2003) Delta 12-oleate desaturase-related enzymes associated with formation of conjugated trans-delta 11, cis-delta 13 double bonds. J Biol Chem 278, 4603-4610.

(64) Martins, B. M., Dobbek, H., Cinkaya, I., Buckel, W., and Messerschmidt, A. (2004) Crystal structure of 4-hydroxybutyryl-CoA dehydratase: radical catalysis involving a [4Fe-4S] cluster and flavin. Proc Natl Acad Sci U S A 101, 15645-15649.

(65) Polet, H., and Levine, L. (1975) Partial purification and characterization of prostaglandin A isomerase from rabbit serum. Arch Biochem Biophys 168, 96-103.

(66) Stumpe, M., Bode, J., Gobel, C., Wichard, T., Schaaf, A., Frank, W., Frank, M., Reski, R., Pohnert, G., and Feussner, I. (2006) Biosynthesis of C9-aldehydes in the moss Physcomitrella patens. Biochim Biophys Acta 1761, 301-312.

(67) Feussner, I., and Wasternack, C. (2002) The lipoxygenase pathway. Annu Rev Plant Biol 53, 275-297.

(68) Kiefer, J. R., Pawlitz, J. L., Moreland, K. T., Stegeman, R. A., Hood, W. F., Gierse, J. K., Stevens, A. M., Goodwin, D. C., Rowlinson, S. W., Marnett, L. J., Stallings, W. C., and Kurumbail, R. G. (2000) Structural insights into the stereochemistry of the cyclooxygenase reaction. Nature 405, 97-101.

(69) Malkowski, M. G., Ginell, S. L., Smith, W. L., and Garavito, R. M. (2000) The productive conformation of arachidonic acid bound to prostaglandin synthase. Science 289, 1933-1937.

(70) Schneider, C., Pratt, D. A., Porter, N. A., and Brash, A. R. (2007) Control of oxygenation in lipoxygenase and cyclooxygenase catalysis. Chem Biol 14, 473-488.

(71) Bach, T. J. (1995) Some new aspects of isoprenoid biosynthesis in plants--a review. Lipids 30, 191-202.

(72) Rohdich, F., Kis, K., Bacher, A., and Eisenreich, W. (2001) The non-mevalonate pathway of isoprenoids: genes, enzymes and intermediates. Curr Opin Chem Biol 5, $535-540$.

(73) Wouters, J., Oudjama, Y., Barkley, S. J., Tricot, C., Stalon, V., Droogmans, L., and Poulter, C. D. (2003) Catalytic mechanism of Escherichia coli isopentenyl diphosphate isomerase involves Cys-67, Glu-116, and Tyr-104 as suggested by crystal structures of complexes with transition state analogues and irreversible inhibitors. J Biol Chem 278, 11903-11908.

(74) Zheng, W., Sun, F., Bartlam, M., Li, X., Li, R., and Rao, Z. (2007) The crystal structure of human isopentenyl diphosphate isomerase at $1.7 \mathrm{~A}$ resolution reveals its catalytic mechanism in isoprenoid biosynthesis. J Mol Biol 366, 1447-1458.

(75) Heipieper, H. J., Meinhardt, F., and Segura, A. (2003) The cis-trans isomerase of unsaturated fatty acids in Pseudomonas and Vibrio: biochemistry, molecular biology 
and physiological function of a unique stress adaptive mechanism. FEMS Microbiol Lett 229, 1-7.

(76) von Wallbrunn, A., Richnow, H. H., Neumann, G., Meinhardt, F., and Heipieper, H. J. (2003) Mechanism of cis-trans isomerization of unsaturated fatty acids in Pseudomonas putida. J Bacteriol 185, 1730-1733.

(77) Partanen, S. T., Novikov, D. K., Popov, A. N., Mursula, A. M., Hiltunen, J. K., and Wierenga, R. K. (2004) The 1.3 A crystal structure of human mitochondrial Delta3-Delta2-enoyl-CoA isomerase shows a novel mode of binding for the fatty acyl group. J Mol Biol 342, 1197-1208.

(78) Mursula, A. M., van Aalten, D. M., Hiltunen, J. K., and Wierenga, R. K. (2001) The crystal structure of delta(3)-delta(2)-enoyl-CoA isomerase. J Mol Biol 309, 845853.

(79) Zhang, D., Liang, X., He, X. Y., Alipui, O. D., Yang, S. Y., and Schulz, H. (2001) Delta 3,5,delta 2,4-dienoyl-CoA isomerase is a multifunctional isomerase. A structural and mechanistic study. J Biol Chem 276, 13622-13627.

(80) Modis, Y., Filppula, S. A., Novikov, D. K., Norledge, B., Hiltunen, J. K., and Wierenga, R. K. (1998) The crystal structure of dienoyl-CoA isomerase at $1.5 \mathrm{~A}$ resolution reveals the importance of aspartate and glutamate sidechains for catalysis. Structure 6, 957-970.

(81) Kim, S. W., Cha, S. S., Cho, H. S., Kim, J. S., Ha, N. C., Cho, M. J., Joo, S., Kim, K. K., Choi, K. Y., and Oh, B. H. (1997) High-resolution crystal structures of delta53 -ketosteroid isomerase with and without a reaction intermediate analogue. Biochemistry 36, 14030-14036.

(82) Cho, H. S., Choi, G., Choi, K. Y., and Oh, B. H. (1998) Crystal structure and enzyme mechanism of Delta 5-3-ketosteroid isomerase from Pseudomonas testosteroni. Biochemistry 37, 8325-8330.

(83) Kraut, D. A., Sigala, P. A., Pybus, B., Liu, C. W., Ringe, D., Petsko, G. A., and Herschlag, D. (2006) Testing electrostatic complementarity in enzyme catalysis: hydrogen bonding in the ketosteroid isomerase oxyanion hole. PLoS Biol 4, e99.

(84) Brock, D. J., Kass, L. R., and Bloch, K. (1967) Beta-hydroxydecanoyl thioester dehydrase. II. Mode of action. J Biol Chem 242, 4432-4440.

(85) Norris, A. T., Matsumura, S., and Bloch, K. (1964) Fatty Acid Synthetase and Beta-Hydroxydecanoyl Coenzyme a Dehydrase from Escherichia Coli. J Biol Chem 239, 3653-3662.

(86) Heath, R. J., and Rock, C. O. (1996) Roles of the FabA and FabZ betahydroxyacyl-acyl carrier protein dehydratases in Escherichia coli fatty acid biosynthesis. J Biol Chem 271, 27795-27801.

(87) Leesong, M., Henderson, B. S., Gillig, J. R., Schwab, J. M., and Smith, J. L. (1996) Structure of a dehydratase-isomerase from the bacterial pathway for biosynthesis of unsaturated fatty acids: two catalytic activities in one active site. Structure 4, 253-264.

(88) Kimber, M. S., Martin, F., Lu, Y., Houston, S., Vedadi, M., Dharamsi, A., Fiebig, K. M., Schmid, M., and Rock, C. O. (2004) The structure of (3R)-hydroxyacyl-acyl carrier protein dehydratase (FabZ) from Pseudomonas aeruginosa. J Biol Chem 279, 52593-52602. 
(89) Fraaije, M. W., and Mattevi, A. (2000) Flavoenzymes: diverse catalysts with recurrent features. Trends Biochem Sci 25, 126-132.

(90) Marrakchi, H., Choi, K. H., and Rock, C. O. (2002) A new mechanism for anaerobic unsaturated fatty acid formation in Streptococcus pneumoniae. J Biol Chem 277, 44809-44816.

(91) Chapman, S. K., Reid G.A., eds. (1999) Flavoprotein Protocols, Vol. 131, Humana Press Inc, New Jersey.

(92) Kaneda, K., Kuzuyama, T., Takagi, M., Hayakawa, Y., and Seto, H. (2001) An unusual isopentenyl diphosphate isomerase found in the mevalonate pathway gene cluster from Streptomyces sp. strain CL190. Proc Natl Acad Sci U S A 98, 932-937.

(93) Steinbacher, S., Kaiser, J., Gerhardt, S., Eisenreich, W., Huber, R., Bacher, A., and Rohdich, F. (2003) Crystal structure of the type II isopentenyl

diphosphate:dimethylallyl diphosphate isomerase from Bacillus subtilis. $J \mathrm{Mol} \mathrm{Biol}$ 329, 973-982.

(94) Hemmi, H., Ikeda, Y., Yamashita, S., Nakayama, T., and Nishino, T. (2004) Catalytic mechanism of type 2 isopentenyl diphosphate:dimethylallyl diphosphate isomerase: verification of a redox role of the flavin cofactor in a reaction with no net redox change. Biochem Biophys Res Commun 322, 905-910.

(95) Rothman, S. C., Helm, T. R., and Poulter, C. D. (2007) Kinetic and spectroscopic characterization of type II isopentenyl diphosphate isomerase from Thermus thermophilus: evidence for formation of substrate-induced flavin species. Biochemistry 46, 5437-5445.

(96) Kittleman, W., Thibodeaux, C. J., Liu, Y. N., Zhang, H., and Liu, H. W. (2007) Characterization and mechanistic studies of type II isopentenyl diphosphate:dimethylallyl diphosphate isomerase from Staphylococcus aureus. Biochemistry 46, 8401-8413.

(97) Buckel, W., and Golding, B. T. (2006) Radical enzymes in anaerobes. Annu Rev Microbiol 60, 27-49.

(98) Muh, U., Cinkaya, I., Albracht, S. P., and Buckel, W. (1996) 4-HydroxybutyrylCoA dehydratase from Clostridium aminobutyricum: characterization of FAD and iron-sulfur clusters involved in an overall non-redox reaction. Biochemistry 35, 11710-11718.

(99) Hornung, E., Krueger, C., Pernstich, C., Gipmans, M., Porzel, A., and Feussner, I. (2005) Production of (10E,12Z)-conjugated linoleic acid in yeast and tobacco seeds. Biochim Biophys Acta 1738, 105-114.

(100) Ausubel, F. M., Brent, R., Kingston, R.E., Moore D.D., Seidman, J.G., Smith, J.A., Struhl, K., eds (1999) Current Protocols in Molecular Biology, John Wiley \& Sons

(101) Schneider, T. R., and Sheldrick, G. M. (2002) Substructure solution with SHELXD. Acta Crystallogr D Biol Crystallogr D58, 1772-1779.

(102) Sheldrick, G. M. (2002) Macromolecular phasing with SHELXE. Z. Kristallogr. 217, 644-650.

(103) Lamzin, V. S., and Wilson, K. S. (1993) Automated refinement of protein models. Acta Cryst D49, 129-147.

(104) Emsley, P., and Cowtan, K. (2004) Coot: model-building tools for molecular graphics. Acta Crystallogr D Biol Crystallogr 60, 2126-2132. 
(105) CCP4. (1994) The Collaborative Computational Project Number 4, suite programs for protein crystallography. Acta Cryst. D50, 760-763.

(106) Brünger, A. T. (1992) Free R value: a novel statistical quantity for assessing the accuracy of crystal structures. Nature 355, 472-475.

(107) Laskowski, R. A., MacArthur, M. W., Moss, D. S., and Thornton, J. M. (1993) PROCHECK: A program to check the stereochemical quality of protein structures. $J$ Appl Cryst 26, 283-291.

(108) Frishman, D., and Argos, P. (1995) Knowledge-based protein secondary structure assignment. Proteins 23, 566-579.

(109) Esnouf, R. M. (1997) An extensively modified version of MOLSCRIPT that includes greatly enhanced coloring capabilities. J Mol Graph 15, 132-134.

(110) Merritt, E. A., and Murphy, M. E. P. (1994) Raster3D Version 2.0 - A program for photorealistic molecular graphics. Acta Cryst D50, 869-873.

(111) Bligh, E. G., and Dyer, W. J. (1959) A rapid method of total lipid extraction and purification. Can J Biochem Physiol 37, 911-917.

(112) Massey, V., and Hemmerich, P. (1978) Photoreduction of flavoproteins and other biological compounds catalyzed by deazaflavins. Biochemistry 17, 9-16.

(113) Frangioni, J. V., and Neel, B. G. (1993) Solubilization and purification of enzymatically active glutathione S-transferase (pGEX) fusion proteins. Anal Biochem 210, 179-187.

(114) Dauter, Z., Dauter, M., and Rajashankar, K. R. (2000) Novel approach to phasing proteins: derivatization by short cryo-soaking with halides. Acta Crystallogr D Biol Crystallogr 56, 232-237.

(115) Pape, T., Schneider, T. R. (2004) HKL2MAP: a graphical user interface for phasing with SHELX programs. J. Appl. Cryst. 37 37, 843-844.

(116) Uson, I., Schmidt, B., von Bulow, R., Grimme, S., von Figura, K., Dauter, M., Rajashankar, K. R., Dauter, Z., and Sheldrick, G. M. (2003) Locating the anomalous scatterer substructures in halide and sulfur phasing. Acta Crystallogr D Biol Crystallogr 59, 57-66.

(117) Dym, O., and Eisenberg, D. (2001) Sequence-structure analysis of FADcontaining proteins. Protein Sci 10, 1712-1728.

(118) Huang, Q., Liu, Q., and Hao, Q. (2005) Crystal structures of Fms1 and its complex with spermine reveal substrate specificity. J Mol Biol 348, 951-959.

(119) Sanders, D. A., Staines, A. G., McMahon, S. A., McNeil, M. R., Whitfield, C., and Naismith, J. H. (2001) UDP-galactopyranose mutase has a novel structure and mechanism. Nat Struct Biol 8, 858-863.

(120) Schalk, I., Zeng, K., Wu, S. K., Stura, E. A., Matteson, J., Huang, M., Tandon, A., Wilson, I. A., and Balch, W. E. (1996) Structure and mutational analysis of Rab GDP-dissociation inhibitor. Nature 381, 42-48.

(121) Gouet, P., Courcelle, E., Stuart, D.I., Metoz, F. . (1999) ESPript: multiple sequence alignments in PostScript. Bioinformatics 15, 305-308.

(122) Kim, J. J., Wang, M., and Paschke, R. (1993) Crystal structures of medium-chain acyl-CoA dehydrogenase from pig liver mitochondria with and without substrate. Proc Natl Acad Sci U S A 90, 7523-7527. 
(123) Gaffney, B. J., Boyington, J. C., Amzel, L. M., Doctor, K. S., Prigge, S. T., and Yuan, S. M. (1995) Lipoxygenase structure and mechanism. Adv Prostaglandin Thromboxane Leukot Res 23, 11-16.

(124) Bornemann, S. (2002) Flavoenzymes that catalyse reactions with no net redox change. Nat Prod Rep 19, 761-772.

(125) Ghisla, S., and Massey, V. (1989) Mechanisms of flavoprotein-catalyzed reactions. Eur J Biochem 181, 1-17.

(126) Christie, W. W. (1998) Gas chromatography-mass spectrometry methods for structural analysis of fatty acids. Lipids 33, 343-353.

(127) Oldham, M. L., Brash, A. R., and Newcomer, M. E. (2005) Insights from the Xray crystal structure of coral 8R-lipoxygenase: calcium activation via a C2-like domain and a structural basis of product chirality. J Biol Chem 280, 39545-39552.

(128) Skrzypczak-Jankun, E., Bross, R. A., Carroll, R. T., Dunham, W. R., and Funk, M. O., Jr. (2001) Three-dimensional structure of a purple lipoxygenase. J Am Chem Soc 123, 10814-10820.

(129) Minor, W., Steczko, J., Stec, B., Otwinowski, Z., Bolin, J. T., Walter, R., and Axelrod, B. (1996) Crystal structure of soybean lipoxygenase L-1 at 1.4 A resolution. Biochemistry 35, 10687-10701.

(130) Gillmor, S. A., Villasenor, A., Fletterick, R., Sigal, E., and Browner, M. F. (1997) The structure of mammalian 15-lipoxygenase reveals similarity to the lipases and the determinants of substrate specificity. Nat Struct Biol 4, 1003-1009.

(131) May, C., Hohne, M., Gnau, P., Schwennesen, K., and Kindl, H. (2000) The Nterminal beta-barrel structure of lipid body lipoxygenase mediates its binding to liposomes and lipid bodies. Eur J Biochem 267, 1100-1109.

(132) Kil, K. S., Cunningham, M. W., and Barnett, L. A. (1994) Cloning and sequence analysis of a gene encoding a 67-kilodalton myosin-cross-reactive antigen of Streptococcus pyogenes reveals its similarity with class II major histocompatibility antigens. Infect Immun 62, 2440-2449.

(133) Lario, P. I., Sampson, N., and Vrielink, A. (2003) Sub-atomic resolution crystal structure of cholesterol oxidase: what atomic resolution crystallography reveals about enzyme mechanism and the role of the FAD cofactor in redox activity. $J$ Mol Biol $326,1635-1650$.

(134) Lim, L., Molla, G., Guinn, N., Ghisla, S., Pollegioni, L., and Vrielink, A. (2006) Structural and kinetic analyses of the H121A mutant of cholesterol oxidase. Biochem $J$ 400, 13-22.

(135) Binda, C., Li, M., Hubalek, F., Restelli, N., Edmondson, D. E., and Mattevi, A. (2003) Insights into the mode of inhibition of human mitochondrial monoamine oxidase B from high-resolution crystal structures. Proc Natl Acad Sci U S A 100, 9750-9755.

(136) Wohlfahrt, G., Witt, S., Hendle, J., Schomburg, D., Kalisz, H. M., and Hecht, H. J. (1999) 1.8 and 1.9 A resolution structures of the Penicillium amagasakiense and Aspergillus niger glucose oxidases as a basis for modelling substrate complexes. Acta Crystallogr D Biol Crystallogr 55, 969-977.

(137) Mattevi, A., Schierbeek, A. J., and Hol, W. G. (1991) Refined crystal structure of lipoamide dehydrogenase from Azotobacter vinelandii at 2.2 A resolution. A comparison with the structure of glutathione reductase. J Mol Biol 220, 975-994. 
(138) Malkowski, M. G., Thuresson, E. D., Lakkides, K. M., Rieke, C. J., Micielli, R., Smith, W. L., and Garavito, R. M. (2001) Structure of eicosapentaenoic and linoleic acids in the cyclooxygenase site of prostaglandin endoperoxide $\mathrm{H}$ synthase-1. J Biol Chem 276, 37547-37555.

(139) Kohno-Murase, J., Iwabuchi, M., Endo-Kasahara, S., Sugita, K., Ebinuma, H., and Imamura, J. (2006) Production of trans-10, cis-12 conjugated linoleic acid in rice. Transgenic Res 15, 95-100.

(140) Tilley, S. J., Skippen, A., Murray-Rust, J., Swigart, P. M., Stewart, A., Morgan, C. P., Cockcroft, S., and McDonald, N. Q. (2004) Structure-function analysis of human [corrected] phosphatidylinositol transfer protein alpha bound to phosphatidylinositol. Structure 12, 317-326.

(141) Gadola, S. D., Zaccai, N. R., Harlos, K., Shepherd, D., Castro-Palomino, J. C., Ritter, G., Schmidt, R. R., Jones, E. Y., and Cerundolo, V. (2002) Structure of human CD1b with bound ligands at $2.3 \mathrm{~A}$, a maze for alkyl chains. Nat Immunol 3, 721-726.

(142) Roderick, S. L., Chan, W. W., Agate, D. S., Olsen, L. R., Vetting, M. W., Rajashankar, K. R., and Cohen, D. E. (2002) Structure of human phosphatidylcholine transfer protein in complex with its ligand. Nat Struct Biol 9, 507-511.

(143) Klinman, J. P. (2006) The role of tunneling in enzyme catalysis of C-H activation. Biochim Biophys Acta 1757, 981-987.

(144) Kohen, A., and Klinman, J. P. (1999) Hydrogen tunneling in biology. Chem Biol 6, R191-198.

(145) Sutcliffe, M. J., and Scrutton, N. S. (2002) A new conceptual framework for enzyme catalysis. Hydrogen tunnelling coupled to enzyme dynamics in flavoprotein and quinoprotein enzymes. Eur J Biochem 269, 3096-3102.

(146) Basran, J., Harris, R. J., Sutcliffe, M. J., and Scrutton, N. S. (2003) H-tunneling in the multiple $\mathrm{H}$-transfers of the catalytic cycle of morphinone reductase and in the reductive half-reaction of the homologous pentaerythritol tetranitrate reductase. $J$ Biol Chem 278, 43973-43982.

(147) Bahnson, B. J., Colby, T. D., Chin, J. K., Goldstein, B. M., and Klinman, J. P. (1997) A link between protein structure and enzyme catalyzed hydrogen tunneling. Proc Natl Acad Sci U S A 94, 12797-12802.

(148) Kim, J. J., and Miura, R. (2004) Acyl-CoA dehydrogenases and acyl-CoA oxidases. Structural basis for mechanistic similarities and differences. Eur J Biochem 271, 483-493.

(149) Ghisla, S., and Thorpe, C. (2004) Acyl-CoA dehydrogenases. A mechanistic overview. Eur J Biochem 271, 494-508.

(150) Huang, Z., Zhang, Q., and Liu, H. W. (2003) Reconstitution of UDPgalactopyranose mutase with 1-deaza-FAD and 5-deaza-FAD: analysis and mechanistic implications. Bioorg Chem 31, 494-502.

(151) Edmondson, D. E., Barman, B., and Tollin, G. (1972) On the importance of the N-5 position in flavin coenzymes. Properties of free and protein-bound 5-deaza analogs. Biochemistry 11, 1133-1138.

(152) Cao, J., Wang,Y.J., Yu,H.D. and Wang,Y.N. (2005) Cloning and expression of linoleate isomerase gene from Lactobacillus acidophilus AS1.1854 and properties of the

recombinant enzyme. seq. ID DQ239438. 
(153) Irmak, S., Dunford, N. T., Gilliland, S. E., Banskalieva, V., and Eisenmenger, M. (2006) Biocatalysis of linoleic acid to conjugated linoleic acid. Lipids 41, 771-776.

(154) Niehaus, W. G., Jr., Kisic, A., Torkelson, A., Bednarczyk, D. J., and Schroepfer, G. J., Jr. (1970) Stereospecific hydration of cis- and trans-9,10-epoxyoctadecanoic acids. J Biol Chem 245, 3802-3809.

(155) Schroepfer, G. J., Jr., Niehaus, W. G., Jr., and McCloskey, J. A. (1970) Enzymatic conversion of linoleic acid to 10D-hydroxy-delta 12-cis-octadecenoic acid. $J$ Biol Chem 245, 3798-3801.

(156) Coakley, M., Ross, R. P., Nordgren, M., Fitzgerald, G., Devery, R., and Stanton, C. (2003) Conjugated linoleic acid biosynthesis by human-derived Bifidobacterium species. J Appl Microbiol 94, 138-145. 


\section{Abbreviations}

10-HH

$10-\mathrm{HO}$

10-HOE

4-BUDH

AA

$\mathrm{ACP}$

CLA

CLnA

CoA

CTI

DECI

DHA

DMAPP

DMOX

DTT

EA

ECI

EDTA

EPA

ER

FA

GSH

GST

IDI

IFN

IKK

IL

INOS

IPP

IPTG

KIE

KSI

LA

LeA

LOX

LPS

MRCA

MW

NF-kB 10-hydroxy-hexadecanoic acid

10-hydroxy-octadecanoic acid

10-hydroxy-(12Z)-octadecaenoic acid

4-hydroxybutyryl-CoA dehydrogenase

arachidonic acid

acyl carrier protein

conjugated linoleic acid

conjugated linolenic acid

coenzyme A

cis-trans isomerase from Pseudomonas sp

di-enoyl CoA isomerase

docosahexaenoic acid

dimethylallyl pyrophosphate

5,5-dimethyloxazoline

dithiothreitol

elaidic acid

enoyl coa isomerase

ethylenediamine tetraacetic acid

eicosapentaenoic acid

endoplasmatic reticulum

fatty acid

gluthathione

gluthathione transferase

isopentenyl diphosphate:dimethylallyl diphosphate isomerase

interferon

IkB kinase

interleukin

indusible nitric oxide synthase

isopentenyl diphosphate

isopropyl-beta-D-thiogalactopyranoside

kinetic isotope effect

ketosteroid isomerase

linoleic acid

linolenic acid

lipoxygenase

lipopolysacharide

Myosin cross reactive antiden

molecular weight

nuclear factor $\mathrm{kB}$ 


$\begin{array}{ll}\text { NLS } & \text { N-lauroyl sarcosin } \\ \text { OA } & \text { oleic acid } \\ \text { ORF } & \text { open reading frame } \\ \text { PA } & \text { palmitoleic acid } \\ \text { PC } & \text { phosphatidylcholine } \\ \text { PCR } & \text { polymerase chain reaction } \\ \text { PEG } & \text { polyethyleneglycol } \\ \text { PFI } & \text { Ptilota filicina isomerase } \\ \text { PGE2 } & \text { prostaglandin E2 } \\ \text { PGHS } & \text { prostaglandin endoperoxide H synthase } \\ \text { PI } & \text { phosphatidylinositol } \\ \text { PL } & \text { phospholipid } \\ \text { PPAR } & \text { peroxisome proliferators activated receptor } \\ \text { RA } & \text { ricinoleic acid } \\ \text { RA } & \text { ricinoleic acid } \\ \text { RMSD } & \text { root mean square deviation } \\ \text { RT } & \text { retention time } \\ \text { SA } & \text { stearic acid } \\ \text { SAD } & \text { single-wavelength anomalous diffraction } \\ \text { SIRAS } & \text { single-isomorphous replacement with anomalous scattering } \\ \text { TAG } & \text { triacylglycerol } \\ \text { TLA } & \text { trilinoleylacylglycerol } \\ \text { TNF } \alpha & \text { tumor necrosis factor alpha } \\ \text { UFA } & \text { unsaturated fatty acids } \\ \text { VA } & \text { vaccenic acid } \\ & \end{array}$




\section{Curriculum vitae}

Alena Liavonchanka

Born on 18.03.1979 in Vysoki Borak, Belarus

2004-present doctoral studies, Dept. of Plant Biochemistry, University of Göttingen

2003-2004 Advanced graduate studies, Grade B.

IMPRS program Molecular Biology, Göttingen.

2002-2003 Visiting scientist, pharmaceutical chemistry and analysis.

University of Liege, Belgium.

2001-2002 Research scientist, Chemistry Dept.

Belarusian State University, Minsk, Belarus.

1996-2001 University studies, Chemistry Dept., with focus on pharmaceutical chemistry and biochemistry. Grade A, diploma with honors.

Belarusian State University, Minsk, Belarus.

1994-1996 High school, Lyceum at Belarusian State University, Grade A.

Minsk, Belarus. 